\title{
Assessing the Limits of Sustainability for the Delépine Reaction
}

\author{
Electronic Supporting Information
}

Andrew Jordan, ${ }^{a *}$ Shanjun Huang, ${ }^{a}$ Helen F. Sneddon ${ }^{b}$ and Andrew Nortcliffe. ${ }^{a}$

a GlaxoSmithKline Carbon Neutral Laboratory for Sustainable Chemistry, Jubilee Campus, University of Nottingham, 6 Triumph Rd, Nottingham NG7 2GA. *Corresponding author E-mail: andrew.jordan@nottingham.ac.uk.

b GlaxoSmithKline, Medicines Research Centre, Gunnels Wood Road, Stevenage, Herts., UK, SG1 2NY. E-mail: helen.f.sneddon@gsk.com; Fax: +44 (0)1438 768302;Tel: +44 (0)1438 763645

\section{Contents}

Assessing the Limits of Sustainability - A Greener Delépine Reaction ....................

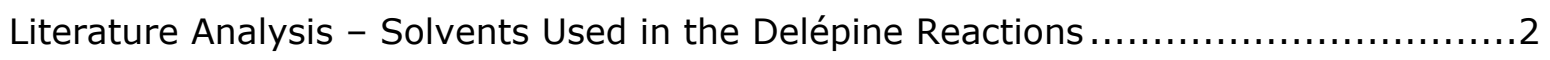

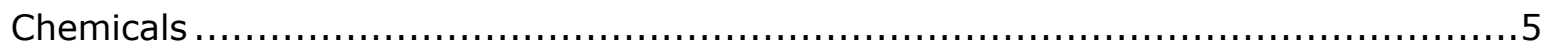

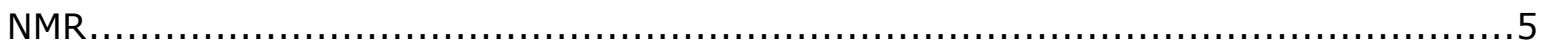

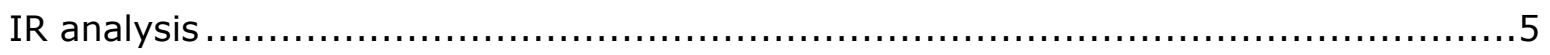

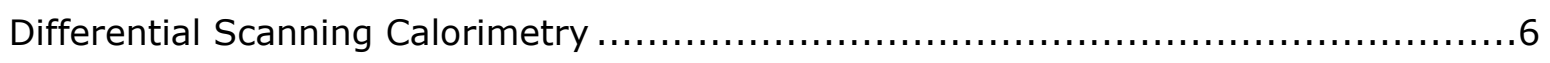

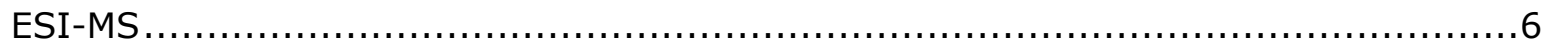

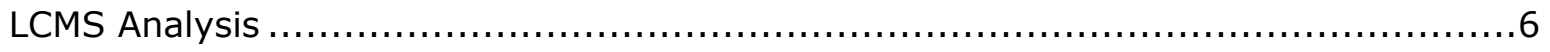

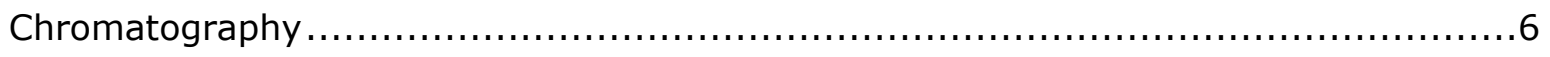

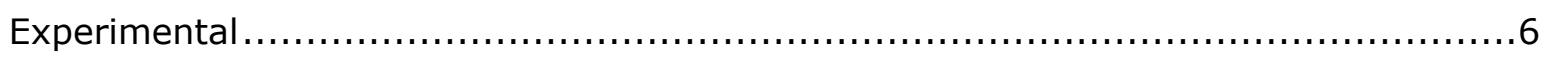

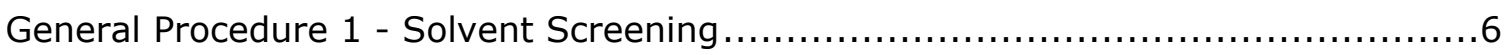

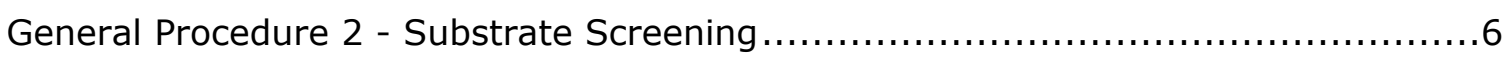

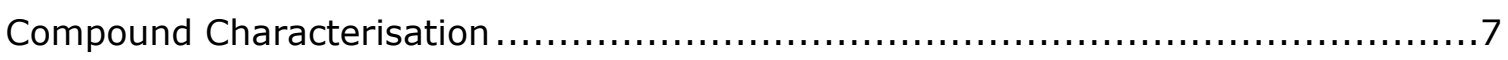

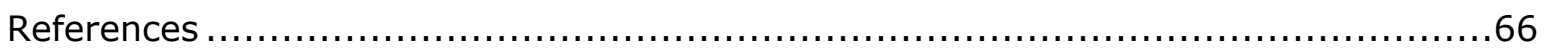

Number of Pages: 66

Number of Figures: 2

Number of Schemes: 1

Number of Tables: 3 


\section{Literature Analysis - Solvents Used in the Delépine Reactions}

Product Search:<smiles>[R]C[N+]12CN3CN(CN(C3)C1)C2</smiles>

Fig. S1: Generic structure search for HMTA salts conducted using SciFinder.

A generic product search was conducted in SciFinder using the structure in Fig. S1. Initially 752 reactions were found, however reaction solvent information cannot be readily compiled from multi-step synthesis extracted from patents. Thus, search results were confined to single step transformations with reported yields to give 221 reactions from 142 publications (including patents) - 33 of which did not have a solvent reported giving a final number of 188 , Table $\mathrm{S} 1$.

Solvent Jul 20, 2020

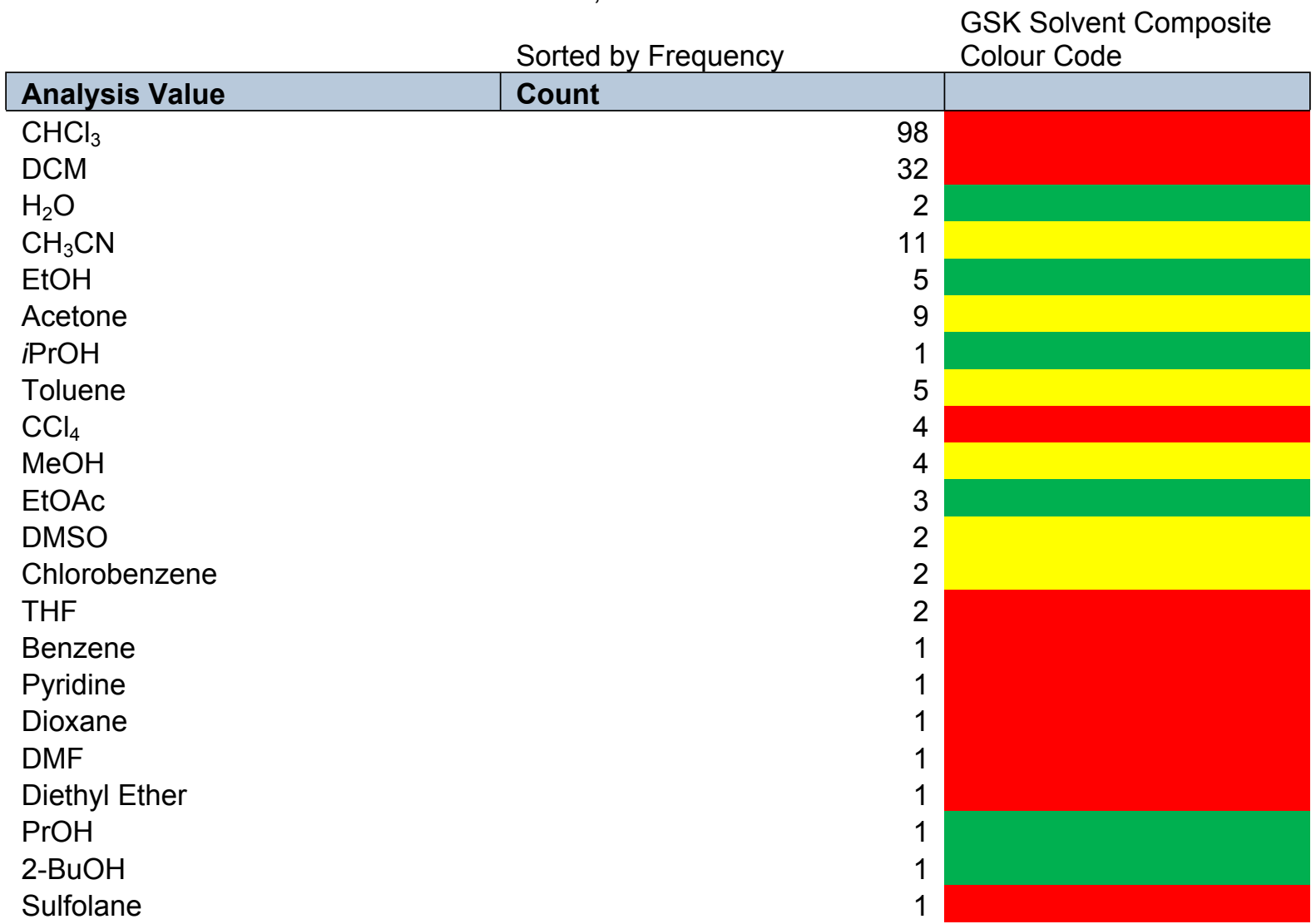

Table S1: SciFinder solvent analysis for Delépine reactions with reported yields. Colour composite: green $=$ few issues, amber $=$ some issues, red $=$ major issues .

13 reactions utilising water were discounted from the initial 16 reported as they were part of another stage of a multi-step synthesis that were not filtered out correctly by SciFinder. Three of the remaining examples were utilised in the synthesis of poly quaternary salts ${ }^{1}$, a strategy that we found unsuitable for our purposes, results of which are reported in the main body of this publication. This left just 2 examples employing water as solvent with reported yields in a mono-alkylation process, Table S1.

EtOAc was reported for use in one journal publication by Li et al. as a MeOH-EtOAc mixture. The product synthesised (with a reported yield) was ethyl HMTA bromide in an $82 \%$ yield. ${ }^{2}$ 
Unfortunately the yields of other HTMA products synthesised in this publication are not reported. The remaining EtOAc examples were reported in patents between the 2001 and 2017. Yields were only reported for two of these examples, Table S2, one of which was an EtOAc-iPrOH binary mixture. Two examples using EtOH were excluded as they were involved in metal complex forming reactions, not alkylations. A further four were excluded as they were reported as part of a poly-alkylated compound ${ }^{3}$, which we found unsuitable for our purposes as exemplified in the main body of this publication. Only four examples included reported yields; other examples are reported in patents but contain no yield data. One example of the use of $i \mathrm{PrOH}$ was reported with a recorded yield of $94 \%$; this example occurs in a patented synthesis of noradrenaline. ${ }^{4}$ No yields were reported for the sole use of $\mathrm{n}-\mathrm{BuOH}^{5}$, and the sole example using n-propanol was for the formation of polyalkylated species $^{3}$. The use of sec-BuOH was exemplified in a patented synthesis of 5aminolevulinate, giving a yield of $75 \%$.

\begin{tabular}{|c|c|c|c|c|}
\hline Solvent & Ref & Year & Yield & $\sum_{N=X^{-}}^{N}$ \\
\hline Water & 1 & 2008 & $99 \%$ & $\underbrace{R=}_{\substack{y_{2} \\
x=C l}}$ \\
\hline $\begin{array}{l}\text { EtOAc-iPrOH } \\
\text { Or Water }\end{array}$ & 6 & 2001 & $98 \%$ & \\
\hline EtOAc & 7 & 2017 & $95 \%$ & $\begin{array}{l}\mathrm{R}= \\
\mathrm{X}=\mathrm{Cl}\end{array}$ \\
\hline EtOAc-MeOH & 2 & 2019 & $82 \%$ & $\begin{array}{l}\mathrm{R}= \\
3 / 2, \\
\mathrm{x}=\mathrm{Br}\end{array}$ \\
\hline EtOH & 8 & 1971 & $95 \%$ & $\begin{array}{l}\mathrm{R}= \\
3 / 2 \\
X=1\end{array}$ \\
\hline EtOH & 9 & 2000 & $75 \%$ & $\begin{array}{l}\mathrm{R}=\mathrm{Br} \\
\mathrm{x}=\mathrm{Br}\end{array}$ \\
\hline EtOH & 10 & 2003 & $69 \%$ & $\begin{array}{l}R= \\
X=I\end{array}$ \\
\hline
\end{tabular}




\begin{tabular}{|c|c|c|c|c|}
\hline EtOH & 11 & 2003 & $97 \%$ & $\begin{array}{l}\mathrm{R}= \\
\mathrm{I}=\mathrm{Br}\end{array}$ \\
\hline EtOH & 12 & 2005 & $85 \%$ & $\begin{array}{l}\mathrm{R}= \\
\mathrm{X}=\mathrm{Cl}\end{array}$ \\
\hline$i \mathrm{PrOH}$ & 4 & 2017 & $94 \%$ & $\begin{array}{l}\mathrm{R}= \\
\mathrm{X}=\mathrm{Cl}\end{array}$ \\
\hline sec-BuOH & 13 & 2019 & $75 \%$ & $\begin{array}{l}\mathrm{R}= \\
\mathrm{X}=\mathrm{Br}\end{array}$ \\
\hline
\end{tabular}

Table S2: Solvent references and yield analysis for green solvents identified in Table S1.

$\Gamma_{\mathrm{N} \sim}^{\mathrm{N}}{ }_{\mathrm{N}}+\mathrm{X} \widehat{\sim}_{\mathrm{GH}}$

Scheme S1: Reaxys structure search using HMTA and a generic alkyl halide as reactants. GH = generic group/hydrogen. $X=$ halide. By using $X-\mathrm{CH}_{2}-\mathrm{GH}$, reactions involving halogenated aromatics, such as the Duff reaction, are effectively excluded.

Similar to the SciFinder product search, a reaction search was conducted using Reaxys according to Scheme S1. This search resulted in 638 reactions found - 239 of which contained no solvent info. This gave a total of 399 reactions. The list was further filtered to remove reactions with no reported yields, and multi-step transformations (which included solvent information from the other steps) to give a total of 117 reactions from 99 publications (including patents). An overview of this Reaxys search is outlined in Table S3 and gives a similar and complementary picture of the solvent usage landscape to that provided by the SciFinder search.

\begin{tabular}{|l|l|l|}
\hline Solvent & Count & $\begin{array}{l}\text { GSK Solvent Composite } \\
\text { Colour Code }\end{array}$ \\
\hline Chloroform & 72 & \\
\hline DCM & 18 & \\
\hline DMF & 11 & \\
\hline Ethanol & 4 & \\
\hline $\mathrm{CH}_{3} \mathrm{CN}$ & 4 & \\
\hline $\mathrm{Toluene}_{\mathrm{CCl}_{4}}$ & 2 & \\
\hline Acetone & 2 & \\
\hline Sulfolane & 2 & \\
\hline iPrOH & 1 & \\
\hline$i$ BuOH & 1 & \\
\hline EtOAc & 1 & \\
\hline
\end{tabular}




\begin{tabular}{|l|l|l|}
\hline Diethyl Ether & 1 & \\
\hline Chlorobenzene & 1 & \\
\hline 1,4-Dioxane & 1 & \\
\hline \multicolumn{2}{|l|}{ Table S3:Solvent usage in the Delépine reaction according to Reaxys, }
\end{tabular}

Table S3:Solvent usage in the Delépine reaction according to Reaxys.

A year of publication SciFinder analysis of Delépine reactions was also conducted using the general product structure depicted in Fig. S1. 752 reactions were discovered and the results are depicted in Fig. S2. What can be seen is varying degrees of popularity with a generally increasing trend in publication numbers towards 2015 , followed by a decrease in use towards 2019. The highest number of publications was observed in 2015 at 82 . In the period of 2009-2019, 40 Delépine containing publications appeared per year on average.

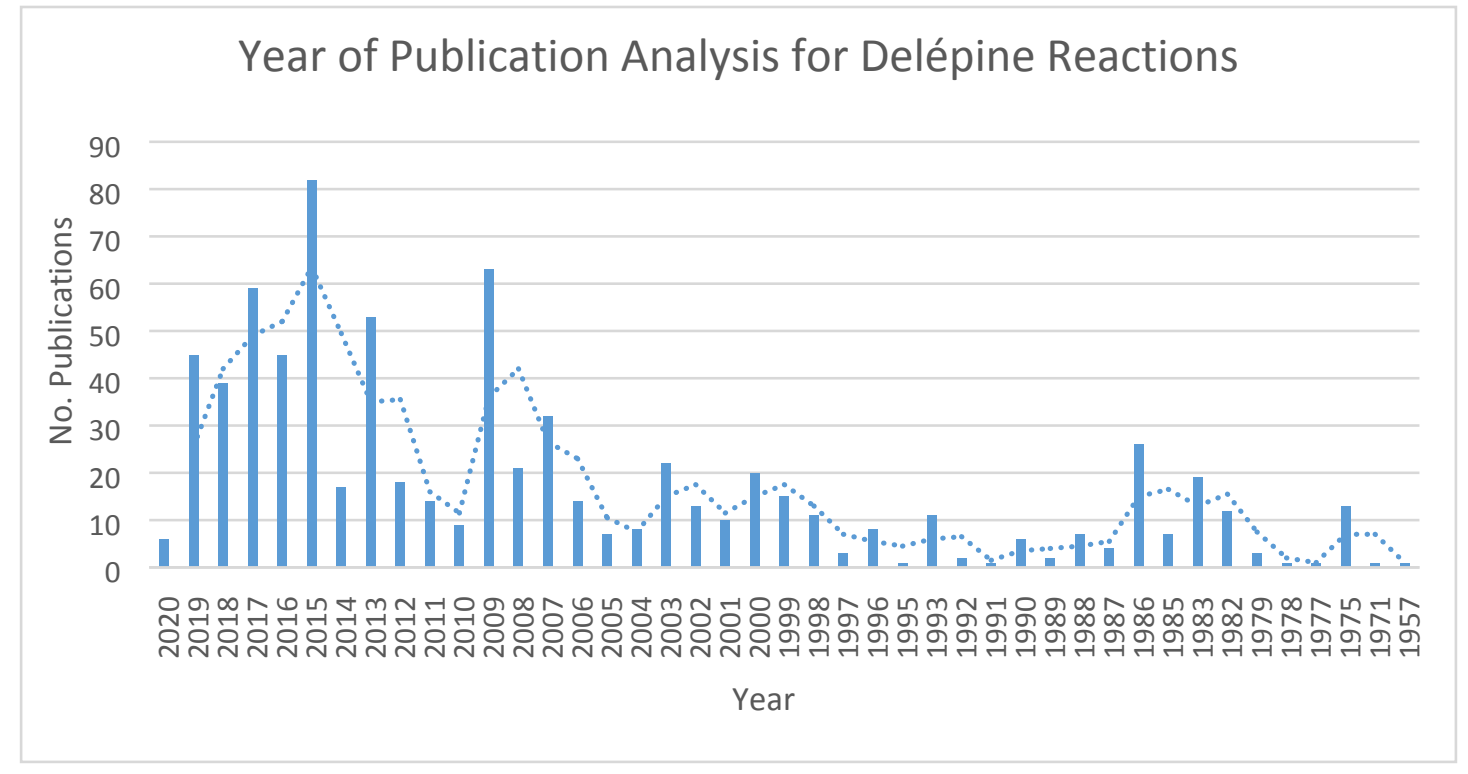

Fig. S2: Year of publication analysis for Delépine reactions with 2-year period moving average trendline.

\section{Chemicals}

All chemicals used were purchased from Sigma-Aldrich, TCI Europe, Acros Organics or Fluorochem. All reagents were used without further purification. Ethyl acetate and chloroform were dried over $3 \AA$ molecular sieves for 24 hours before use. All other solvents were from an Acroseal Extra Dry preparation. Aluminium-backed Silicagel $60 \mathrm{~F}_{254}$ plates from E. Merck were used for thin layer chromatography.

NMR

The majority of NMR analysis was performed on a Bruker AC $400 \mathrm{MHz}$ spectrometer operating at $400 \mathrm{MHz}$ for ${ }^{1} \mathrm{H}$ NMR and $101 \mathrm{MHz}$ for ${ }^{13} \mathrm{C}$ NMR. Samples were run in deuterated chloroform $\left(\mathrm{CDCl}_{3}\right)$ or deuterated dimethyl sulfoxide (DMSO) where appropriate. A $500 \mathrm{MHz}$ Bruker spectrometer, operating at $500 \mathrm{MHz}$ for ${ }^{1} \mathrm{H}$ NMR and 126 $\mathrm{MHz}$ for ${ }^{13} \mathrm{C}$ NMR was also used for analysis of some examples. All chemical shifts are reported in parts per million (ppm), are relative to the internal standard TMS and coupling constants $(J)$ are measured in Hertz $(\mathrm{Hz})$. Multiplicity is stated as follows: s-singlet, ddoublet, t-triplet, q-quartet, dd-doublet of doublets, dt-doublet of triplets, dq-doublet of quartets, tt-triplet of triplets, tq-triplet of quartets, ddd-doublet of doublet of doublets, mmultiplet, bs-broad singlet.

IR analysis

All IR analysis was carried out on a Bruker Alpha Platinum FT-IR spectrometer with ATR. 


\section{Differential Scanning Calorimetry}

DSC measurements were carried out using a TA-instruments Discovery DSC under an $\mathrm{N}_{2}$ atmosphere using a linear heating ramp from $25^{\circ} \mathrm{C}$ to $250^{\circ} \mathrm{C}$ heating at a rate of $10^{\circ} \mathrm{C}$ per minute. Samples were prepared using an aluminium Tzero pan and lid (non hermetic).

\section{ESI-MS}

High resolution mass spectrometry (HRMS) with accurate mass measurement to four decimal places was obtained for all new compounds described. Mass spectra were recorded in both positive and negative electrospray ionisation mode on a Bruker ESI MicroTOF mass spectrometer.

\section{LCMS Analysis}

LCMS for reaction monitoring used an Agilent 1200 system, with a standard gradient elution method: Waters XBridge $2.1 \times 30 \mathrm{~mm}$ column, stationary phase $\mathrm{C}_{18} 3.5 \mu \mathrm{m}, 0.8$ $\mathrm{mL} / \mathrm{min}$ flow rate, detection at $254 \mathrm{~nm}$; elution with 5-95\% MeCN in $0.1 \%$ aqueous ammonia over 4 minutes. Low resolution mass spectrometry was recording using an Agilent G6120B single quadrupole instrument running in MM-ES+APCI ionisation mode.

\section{Chromatography}

A Biotage SP4 was used for the chromatographic purification of compound 29 using a cyclohexane:ethyl acetate gradient elution system with manual fraction collection. UV detector wavelength was set at $254 \mathrm{~nm}$. Interchim $30 \mu \mathrm{m}$ silica gel prepacked flash chromatography cartridges were used for this chromatographic separation.

\section{Experimental}

\section{General Procedure 1 - Solvent Screening}

To a stirred solution of hexamethylenetetramine $(700 \mathrm{mg}, 5.0 \mathrm{mmol})$ in a solvent of choice $(10 \mathrm{~mL})$ was added benzyl bromide $(594 \mu \mathrm{L}, 5.0 \mathrm{mmol})$. The resulting suspension was then heated to $60{ }^{\circ} \mathrm{C}$ for two hours. After two hours the reaction mixture was allowed to cool to room temperature and filtered. The filter cake was washed with isopropanol $(1 \times$ $5 \mathrm{~mL})$ and EtOAc $(2 \times 5 \mathrm{~mL})$ and dried under high vacuum for 24 hours to give the desired product.

\section{General Procedure 2 - Substrate Screening}

To a stirred solution of hexamethylenetetramine $(700 \mathrm{mg}, 5.0 \mathrm{mmol})$ in solvent of choice $(10 \mathrm{~mL})$ was added the appropriate alkyl/aryl bromide $(5.0 \mathrm{mmol})$. The resulting suspension was then heated to $60^{\circ} \mathrm{C}$ for 16 hours. After 16 hours the reaction mixture was allowed to cool to room temperature and filtered. The filter cake was washed with EtOH $(1 \times 25 \mathrm{~mL})$ and EtOAc $(1 \times 25 \mathrm{~mL})$ and dried under high vacuum for 24 hours to give the desired product. 


\section{Compound Characterisation}

1-Benzyl-1,3,5,7-tetraazaadamantan-1-ium bromide $\mathbf{3}$<smiles>BrC12CN3CN(CN(C3)C1)C2</smiles>

1-Benzyl-1,3,5,7-tetraazaadamantan-1-ium bromide was synthesised according to General Procedure 1 using DMC as solvent. Yield: $99 \%(1.55 \mathrm{~g}, 4.98 \mathrm{mmol})$.

Chemical Formula: $\mathrm{C}_{13} \mathrm{H}_{19} \mathrm{BrN}_{4}$

Molecular Weight: $311.23 \mathrm{gmol}^{-1}$

Appearance: White solid

$\mathrm{T}_{\mathrm{m}}{ }^{\circ} \mathrm{C}: 158^{\circ} \mathrm{C}$.

${ }^{1} \mathrm{H}$ NMR $\left(400 \mathrm{MHz}, \mathrm{D}_{2} \mathrm{O}\right) \delta=7.67-7.48(\mathrm{~m}, 5 \mathrm{H}), 5.13(\mathrm{t}, \mathrm{J}=1.1 \mathrm{~Hz}, 6 \mathrm{H}), 4.76-4.67$ $(\mathrm{m}, 3 \mathrm{H}), 4.56-4.47(\mathrm{~m}, 3 \mathrm{H}), 4.17(\mathrm{~s}, 2 \mathrm{H})$.

${ }^{13} \mathrm{C}$ NMR $\left(101 \mathrm{MHz}, \mathrm{D}_{2} \mathrm{O}\right) \delta=132.4,130.9,129.4,124.2,78.0,70.0,61.1$.

FT-ATR max 2975, 2892, 1461, 1268, 1226, 1109, 1039, 1001, 930, 816, 757, 703, 650, 499.

HRMS $m / z$ (ESI) ${ }^{+}$calc. for $\mathrm{C}_{13} \mathrm{H}_{19} \mathrm{~N}_{4}[\mathrm{M}]+$ requires 231.1604 , found 231.1603 .

${ }^{1} \mathrm{H}-\mathrm{NMR}$ was not in agreement with the literature quoted by Wu et al. ${ }^{9}$ who quote

"1H-NMR $\left(\mathrm{D}_{2} \mathrm{O}\right) \delta 7.7(\mathrm{~m}, 5 \mathrm{H}), 4.60(\mathrm{~s}, 2 \mathrm{H}), 3.9(\mathrm{~s}, 6 \mathrm{H}), 3.73(\mathrm{~s}, 6 \mathrm{H}) "$

${ }^{1} \mathrm{H}$ NMR $\left(400 \mathrm{MHz}, \mathrm{D}_{2} \mathrm{O}\right)$ 


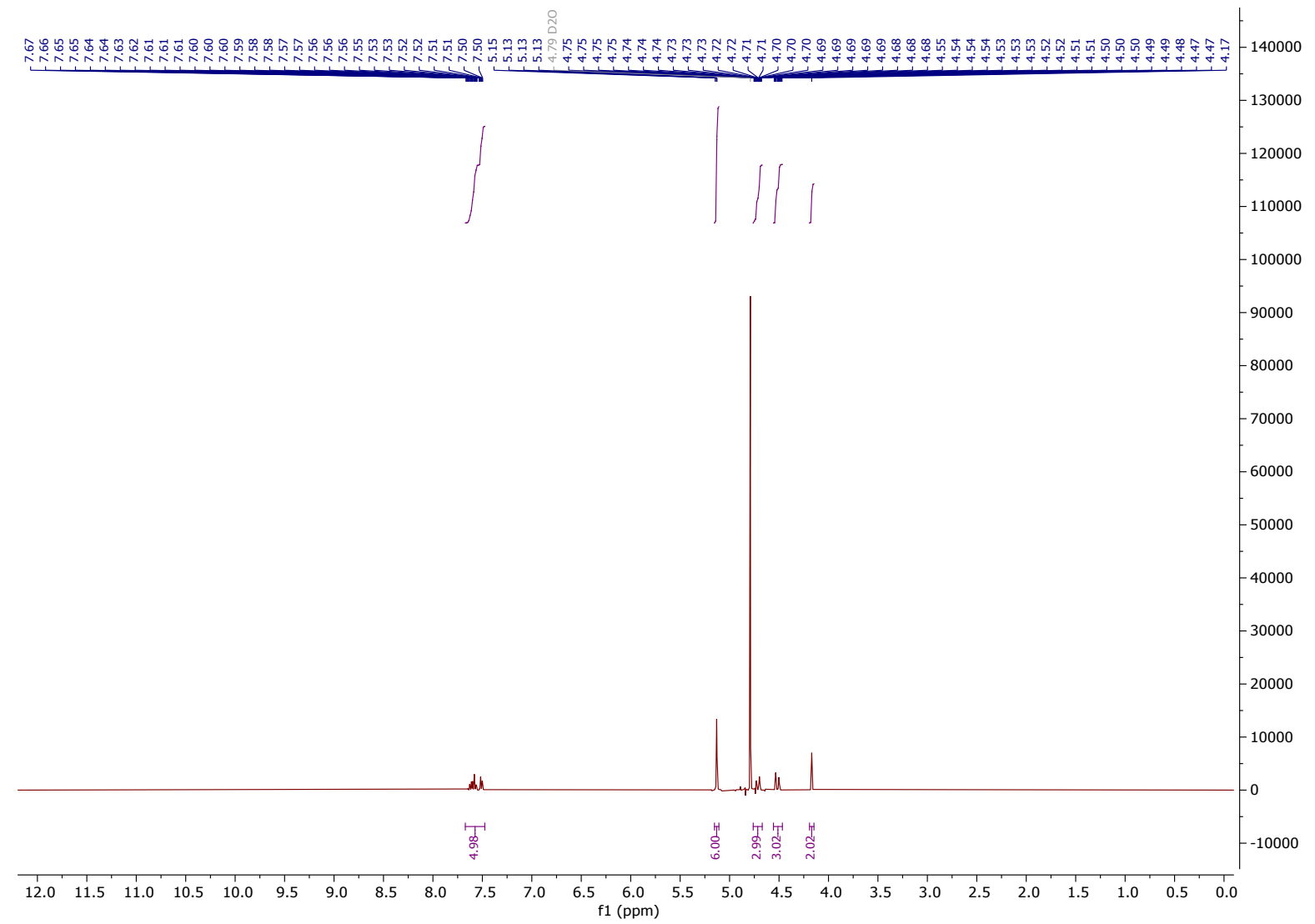

${ }^{13} \mathrm{C}$ NMR $\left(101 \mathrm{MHz}, \mathrm{D}_{2} \mathrm{O}\right)$

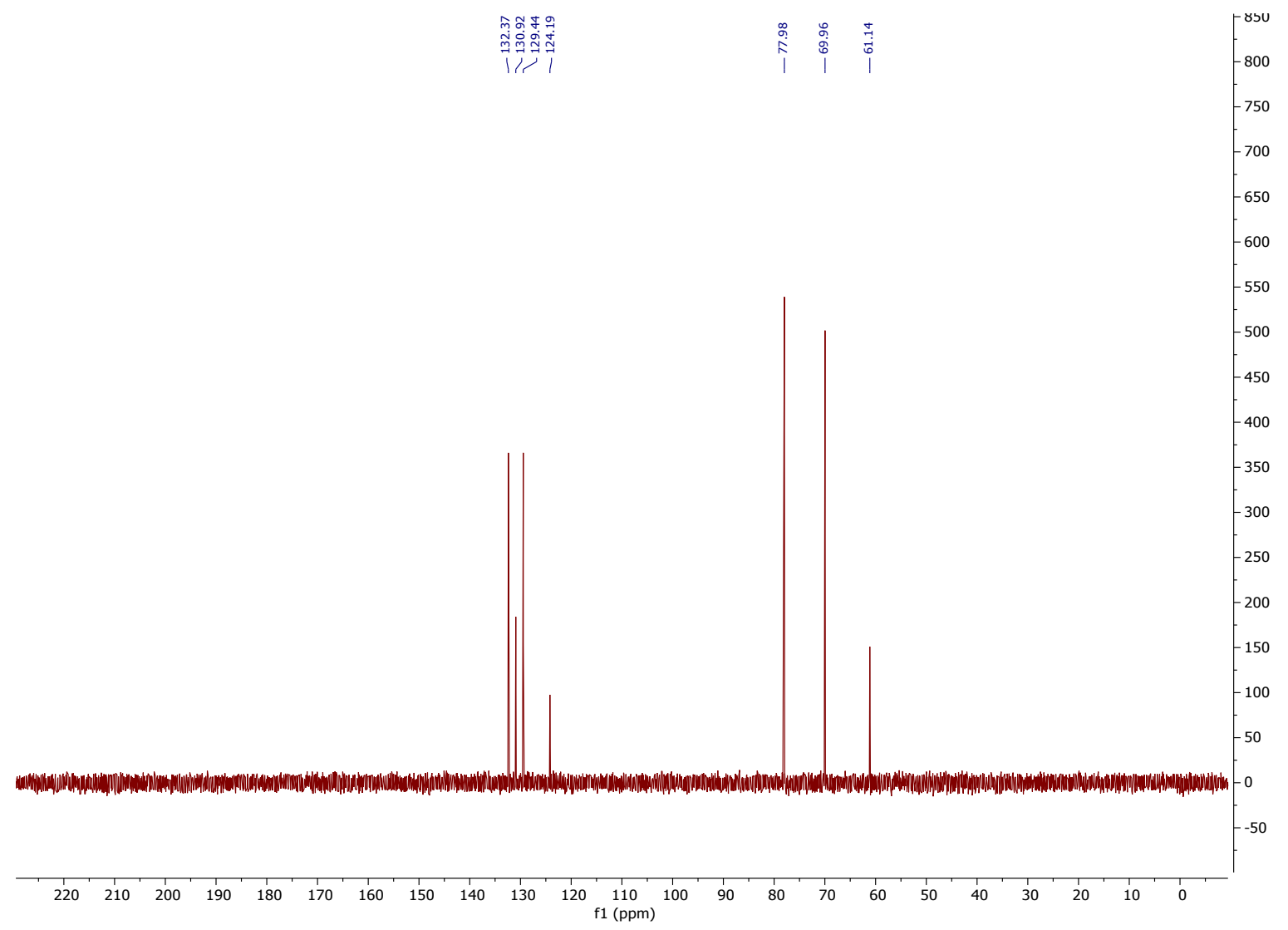


1-(3-Phenylpropyl)-1,3,5,7-tetraazaadamantan-1-ium bromide $\mathbf{5}$<smiles>BrCCCc1ccccc1</smiles>

1-(3-Phenylpropyl)-1,3,5,7-tetraazaadamantan-1-ium bromide was synthesised according to General Procedure 2 using hexamethylenetetramine $(420 \mathrm{mg}, 3.0 \mathrm{mmol})$ and 1-bromo-3-phenylpropane $(456 \mu \mathrm{l}, 3.0 \mathrm{mmol})$. Yield: $54 \%(550 \mathrm{mg}, 1.62 \mathrm{mmol})$.

Chemical Formula: $\mathrm{C}_{15} \mathrm{H}_{23} \mathrm{BrN}_{4}$

Molecular Weight: $339.28 \mathrm{gmol}^{-1}$

Appearance: White solid

${ }^{1} \mathrm{H}$ NMR $\left(400 \mathrm{MHz}, \mathrm{D}_{2} \mathrm{O}\right) \delta=7.49-7.26(\mathrm{~m}, 5 \mathrm{H}), 5.08(\mathrm{~d}, \mathrm{~J}=1.0 \mathrm{~Hz}, 6 \mathrm{H}), 4.76-4.70$

$(\mathrm{m}, 3 \mathrm{H}), 4.59-4.52(\mathrm{~m}, 3 \mathrm{H}), 2.96-2.88(\mathrm{~m}, 2 \mathrm{H}), 2.74(\mathrm{t}, \mathrm{J}=7.2 \mathrm{~Hz}, 2 \mathrm{H}), 2.13-2.03$ $(\mathrm{m}, 2 \mathrm{H})$.

${ }^{13} \mathrm{C}$ NMR $\left(101 \mathrm{MHz}, \mathrm{D}_{2} \mathrm{O}\right) \delta=140.0,128.9,128.6,126.7,78.2,70.1,56.5,31.7,20.9$.

FT-ATR $v_{\max } 2977,2887,1497,1462,1393,1307,1262,1239,1152,1122,1079,1031$, $996,946,869,817,757,726,697,655,501,468$.

HRMS $m / z$ (ESI) ${ }^{+}$calc. for $\mathrm{C}_{15} \mathrm{H}_{23} \mathrm{~N}_{4}[\mathrm{M}]+$ requires 259.1917 , found 259.1914. 
${ }^{1} \mathrm{H}$ NMR $\left(400 \mathrm{MHz}, \mathrm{D}_{2} \mathrm{O}\right)$

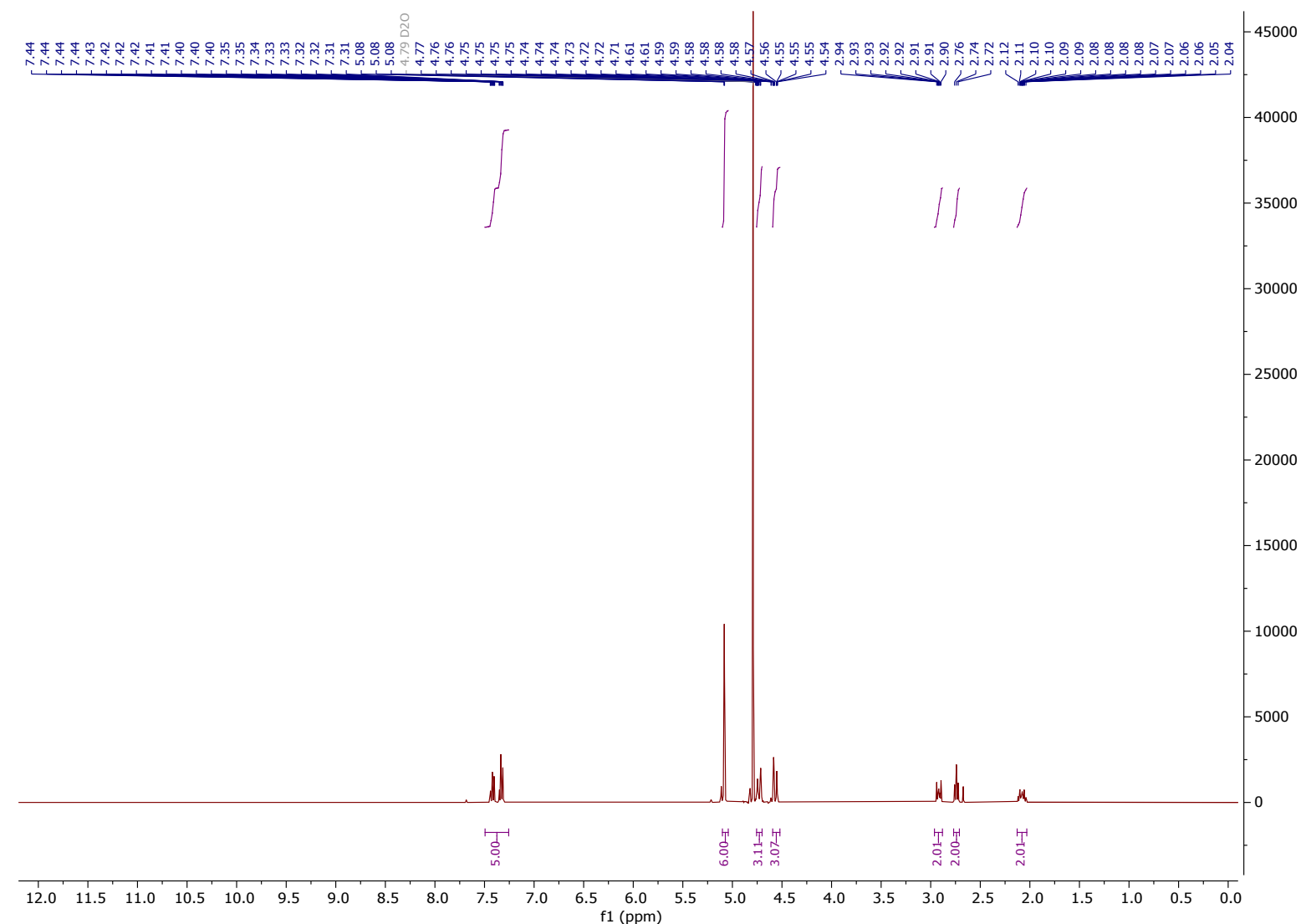

${ }^{13} \mathrm{C}$ NMR $\left(101 \mathrm{MHz}, \mathrm{D}_{2} \mathrm{O}\right)$

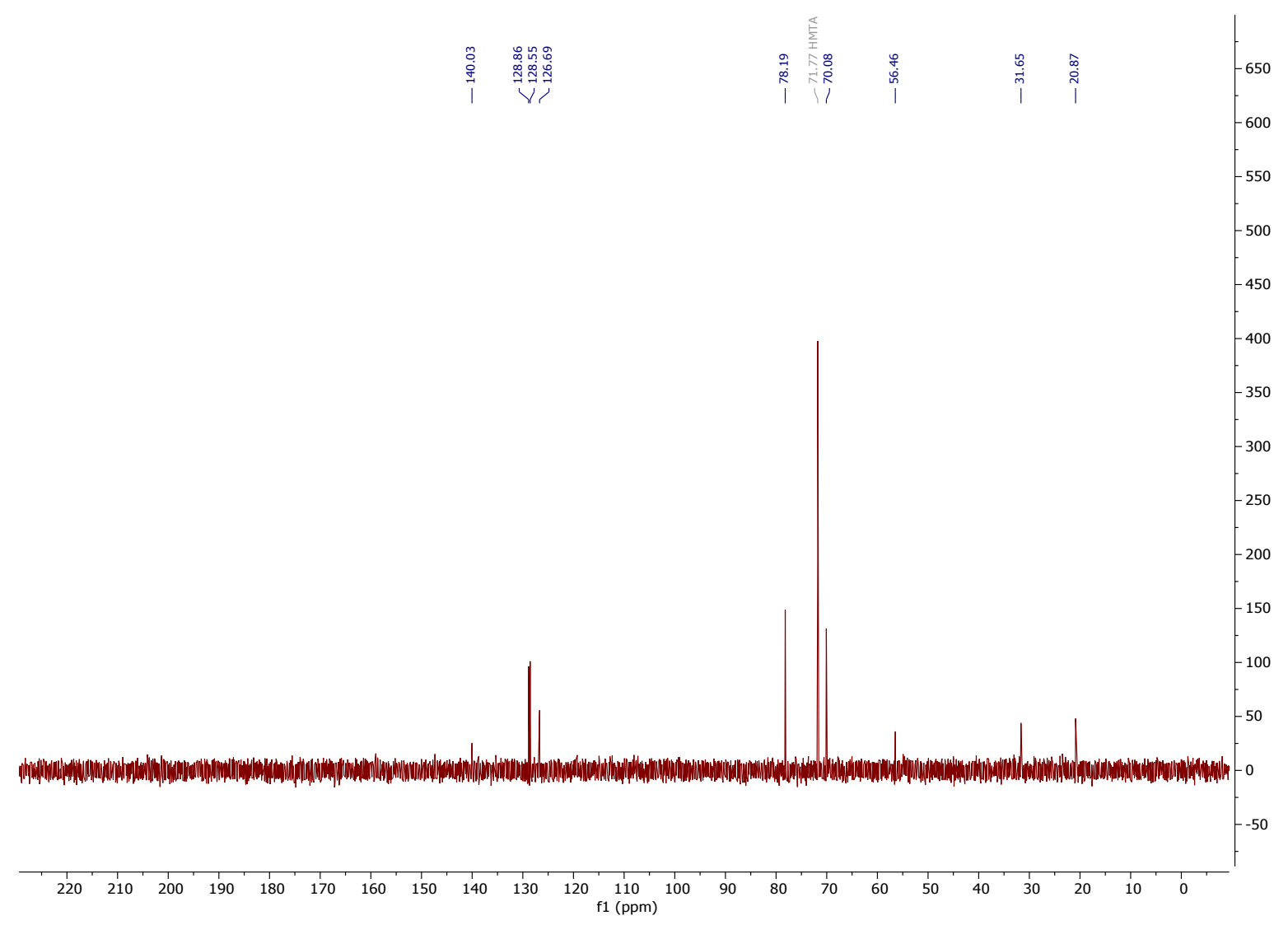


1-(4-Phenylbutyl)-1,3,5,7-tetraazaadamantan-1-ium bromide 6<smiles>BrCCCCc1ccccc1</smiles>

1-(4-Phenylbutyl)-1,3,5,7-tetraazaadamantan-1-ium bromide was synthesised according to General Procedure 2 using hexamethylenetetramine $(420 \mathrm{mg}, 4.7 \mathrm{mmol})$ and 1-bromo4-phenylbutane ( $825 \mu \mathrm{l}, 4.7 \mathrm{mmol})$. Yield: 39\% (621 mg, $1.83 \mathrm{mmol}$ ).

Chemical Formula: $\mathrm{C}_{16} \mathrm{H}_{25} \mathrm{BrN}_{4}$

Molecular Weight: $353.31 \mathrm{gmol}^{-1}$

Appearance: White solid

${ }^{1} \mathrm{H}$ NMR $\left(400 \mathrm{MHz}, \mathrm{D}_{2} \mathrm{O}\right) \delta=7.38-7.28(\mathrm{~m}, 2 \mathrm{H}), 7.26-7.17(\mathrm{~m}, 3 \mathrm{H}), 4.98(\mathrm{~s}, 6 \mathrm{H}), 4.66$ $-4.61(\mathrm{~m}, 3 \mathrm{H}), 4.51-4.44(\mathrm{~m}, 3 \mathrm{H}), 2.87-2.79(\mathrm{~m}, 2 \mathrm{H}), 2.70-2.55(\mathrm{~m}, 2 \mathrm{H}), 1.68-$ $1.57(\mathrm{~m}, 4 \mathrm{H})$.

${ }^{13} \mathrm{C}$ NMR $\left(101 \mathrm{MHz}, \mathrm{D}_{2} \mathrm{O}\right) \delta=141.5,128.7,128.6,126.2,78.1,70.1,57.0,34.1,27.5$, 18.6.

FT-ATR $v_{\max } 2977,2887,1497,1462,1393,1307,1262,1239,1152,1122,1079,1031$, $996,946,869,817,757,726,697,655,501,468$.

$m / z(E S I)+$ calc. for $\mathrm{C}_{16} \mathrm{H}_{25} \mathrm{~N}_{4}[\mathrm{M}]+$ requires 273.2074, found 273.2074. 
${ }^{1} \mathrm{H}$ NMR $\left(400 \mathrm{MHz}, \mathrm{D}_{2} \mathrm{O}\right)$

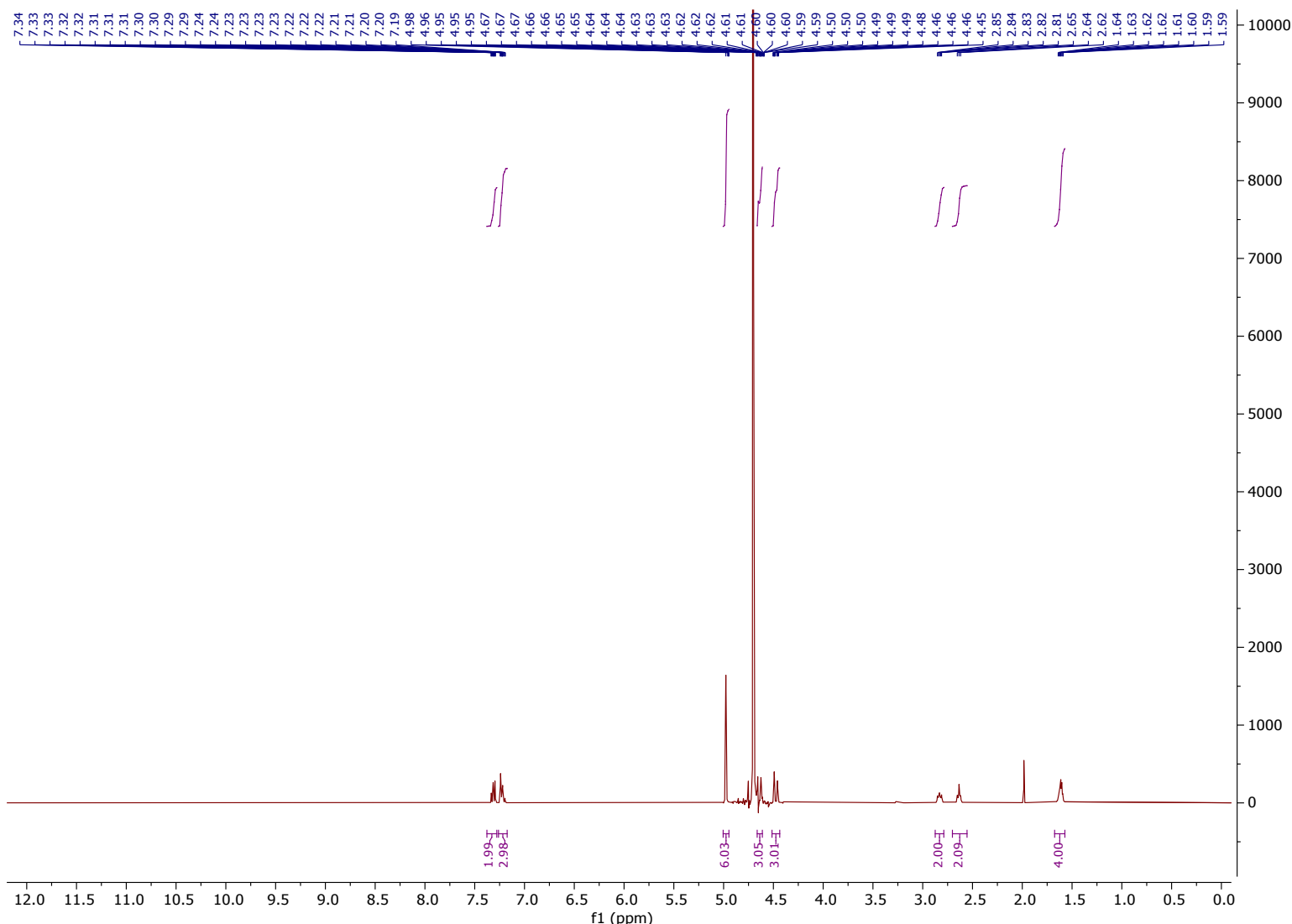

${ }^{13} \mathrm{C}$ NMR $\left(101 \mathrm{MHz}, \mathrm{D}_{2} \mathrm{O}\right)$

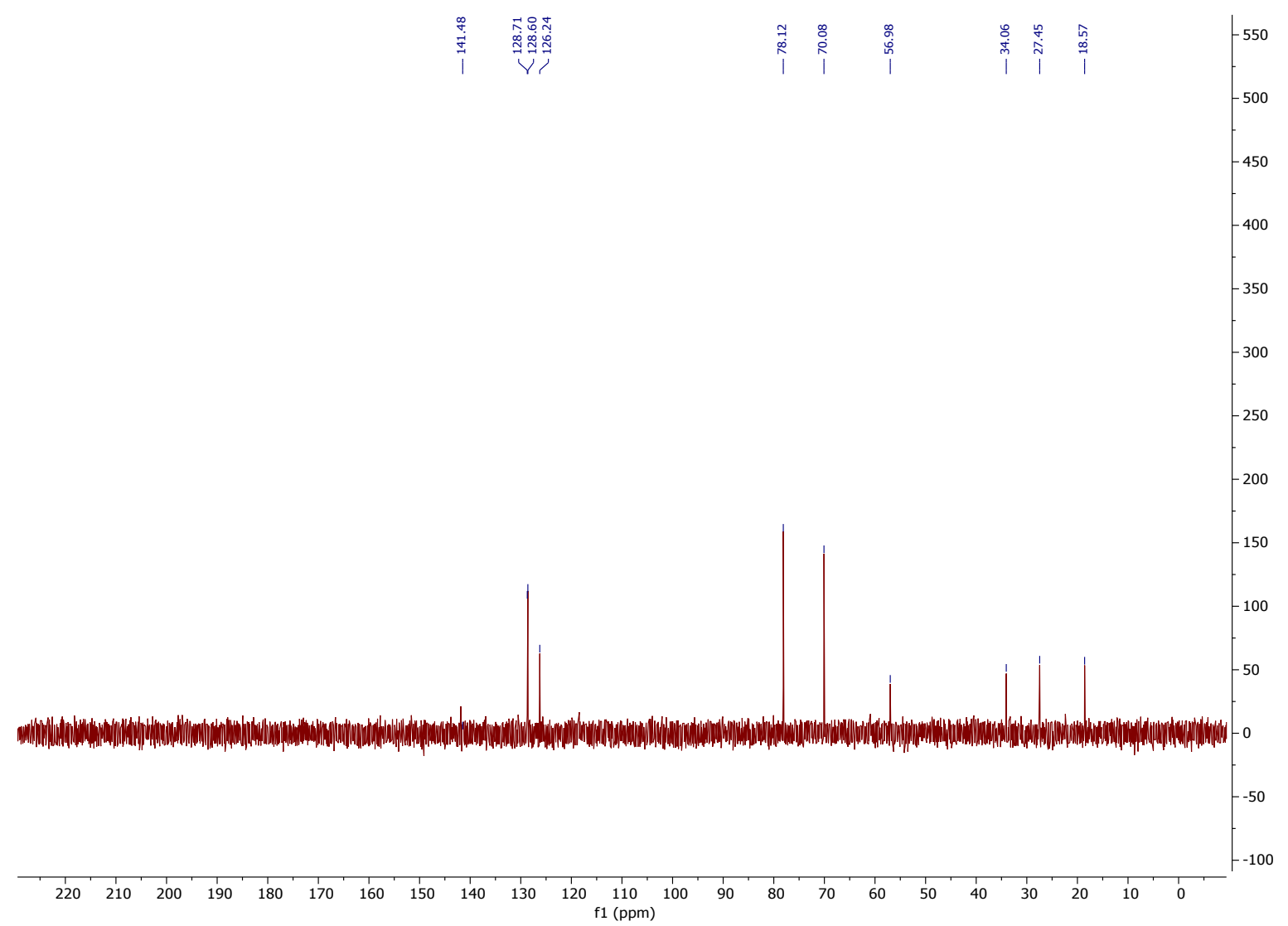


1-(5-Ethoxy-5-oxopentyl)-1,3,5,7-tetraazaadamantan-1-ium bromide 7<smiles>CCOC(=O)CCCC[N+]12CN3CN(CN(C3)C1)C2</smiles>

1-(5-Ethoxy-5-oxopentyl)-1,3,5,7-tetraazaadamantan-1-iumbromide was synthesised according to General Procedure 2 using hexamethylenetetramine ( $701 \mathrm{mg}, 5.0 \mathrm{mmol}$ ) and ethyl 5-bromopentanoate $(791 \mu \mathrm{L}, 5.0 \mathrm{mmol})$. Crude mass recovery after filtration: 1.04 g. Purity by ${ }^{1} \mathrm{H}-\mathrm{NMR}: 82.9 \%$.

Yield: 49\% (862 mg, $2.47 \mathrm{mmol})$.

Chemical Formula: $\mathrm{C}_{13} \mathrm{H}_{25} \mathrm{BrN}_{4} \mathrm{O}_{2}$

Molecular Weight: $349.27 \mathrm{gmol}^{-1}$

Appearance: White solid

${ }^{1} \mathrm{H}$ NMR $\left(400 \mathrm{MHz}, \mathrm{D}_{2} \mathrm{O}\right) \delta=5.13(6 \mathrm{H}, \mathrm{s}), 4.76(3 \mathrm{H}, \mathrm{d}, \mathrm{J} 12.5), 4.63-4.56(3 \mathrm{H}, \mathrm{m}), 4.19$ $(2 \mathrm{H}, \mathrm{q}, \mathrm{J} 7.2), 3.01-2.92(2 \mathrm{H}, \mathrm{m}), 2.48(2 \mathrm{H}, \mathrm{t}, \mathrm{J} 7.2), 1.89-1.72(2 \mathrm{H}, \mathrm{m}), 1.73-1.58$ $(2 \mathrm{H}, \mathrm{m}), 1.27(3 \mathrm{H}, \mathrm{t}, \mathrm{J} 7.2)$.

${ }^{13} \mathrm{C}$ NMR $\left(101 \mathrm{MHz}, \mathrm{D}_{2} \mathrm{O}\right) \delta=175.9,78.2,70.1,61.8,56.7,33.0,21.4,18.8,13.3$.

HRMS $m / z$ (ESI) ${ }^{+}$calcd. for $\mathrm{C}_{13} \mathrm{H}_{25} \mathrm{~N}_{4} \mathrm{O}_{2}[\mathrm{M}]^{+}$required 269.1972, found 269.1987 
${ }^{1} \mathrm{H}$ NMR $\left(400 \mathrm{MHz}, \mathrm{D}_{2} \mathrm{O}\right)$

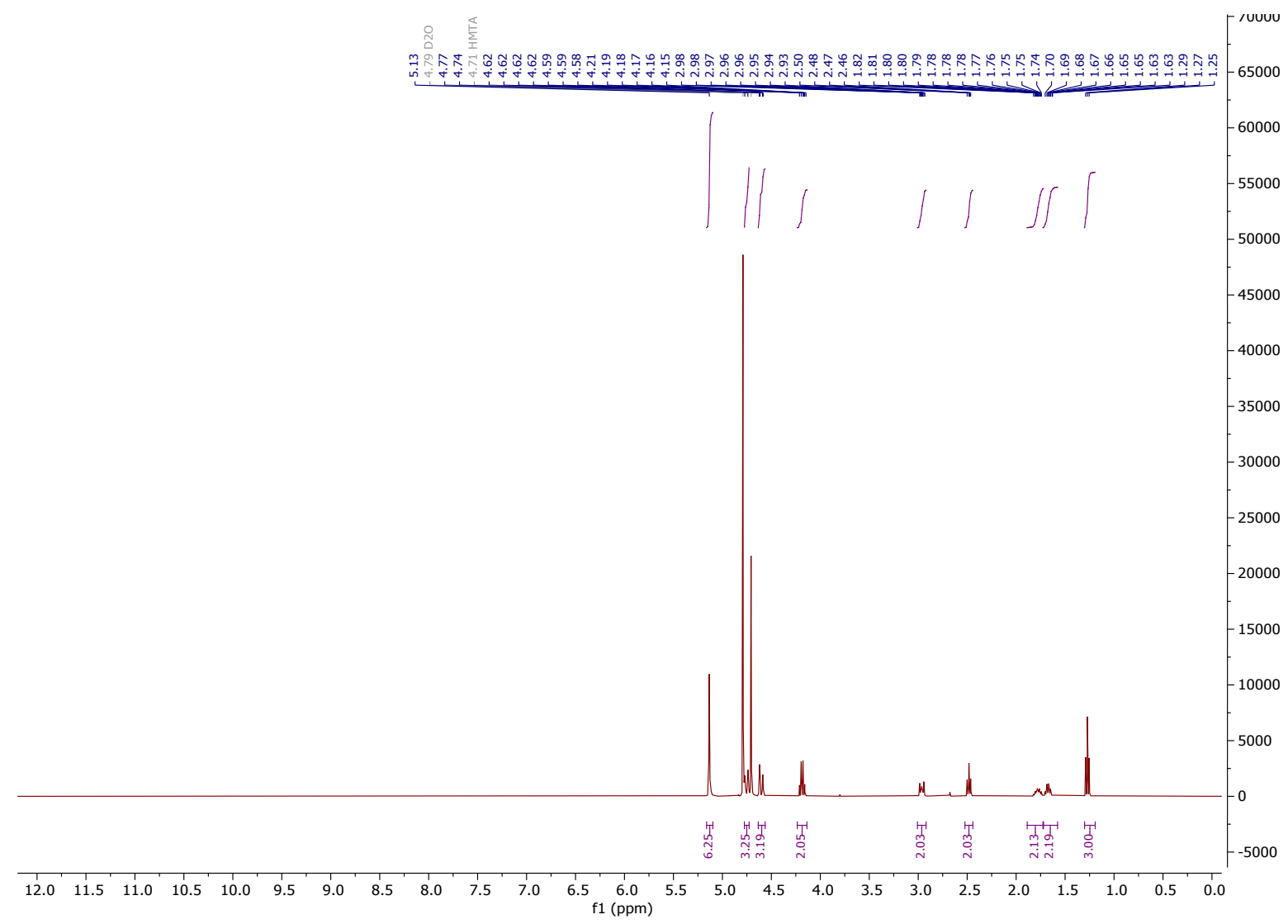

${ }^{13} \mathrm{C}$ NMR $\left(101 \mathrm{MHz}, \mathrm{D}_{2} \mathrm{O}\right)$

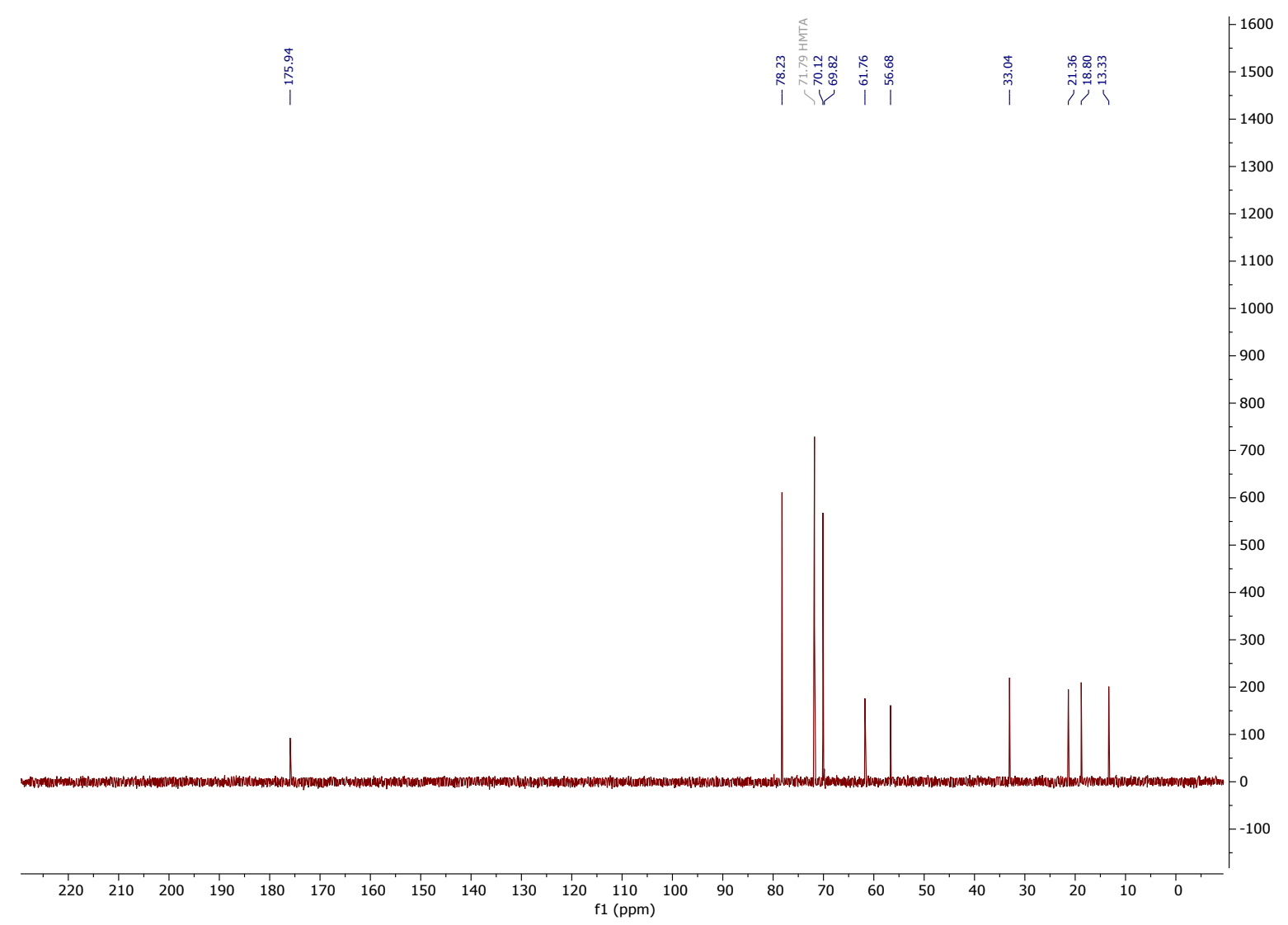


1-(Pent-4-en-1-yl)-1,3,5,7-tetraazaadamantan-1-ium bromide 8

$\mathrm{Br}^{-}$<smiles>C=CCCC[N+]12CN3CN(CN(C3)C1)C2</smiles>

1-(Pent-4-en-1-yl)-1,3,5,7-tetraazaadamantan-1-ium bromide was synthesised according to General Procedure 2 using hexamethylenetetramine $(701 \mathrm{mg}, 5.0 \mathrm{mmol}$ ) and 5-bromo1 -pentene $(592 \mu \mathrm{L}, 5.0 \mathrm{mmol})$. Crude mass recovery after filtration $810 \mathrm{mg}$. Purity by ${ }^{1} \mathrm{H}-$ NMR: 65.5 wt.\%.

Yield: $37 \%$ (531 mg, $1.83 \mathrm{mmol})$.

Chemical Formula: $\mathrm{C}_{11} \mathrm{H}_{21} \mathrm{BrN}_{4}$

Molecular Weight: $289.22 \mathrm{gmol}^{-1}$

Appearance: White solid

${ }^{1} \mathrm{H}$ NMR $\left(400 \mathrm{MHz}, \mathrm{D}_{2} \mathrm{O}\right) \delta=5.94(\mathrm{ddt}, J=17.0,10.3,6.7 \mathrm{~Hz}, 1 \mathrm{H}), 5.25-5.15(\mathrm{~m}, 8 \mathrm{H})$, $4.83(\mathrm{~d}, J=13.2 \mathrm{~Hz}, 3 \mathrm{H}), 4.68(\mathrm{~d}, J=13.2 \mathrm{~Hz}, 3 \mathrm{H}), 3.06-2.95(\mathrm{~m}, 2 \mathrm{H}), 2.23(\mathrm{qt}, J=$ $6.9,1.4 \mathrm{~Hz}, 2 \mathrm{H}), 1.99-1.86(\mathrm{~m}, 2 \mathrm{H})$.

${ }^{13} \mathrm{C}$ NMR $\left(101 \mathrm{MHz}, \mathrm{D}_{2} \mathrm{O}\right) \delta=136.5,116.3,78.2,70.1,56.7,29.8,18.3$.

FT-ATR $v_{\max }$ 2981, 2943, 2894, 641, 1463, 1396, 1365, 1300, 1264 1238, 1125, 1039, 995, 954, 922, 885, 816, 782, 750, 708, 651, 579, 498.

HRMS $m / z(E S I)+$ calcd. for $\mathrm{C}_{11} \mathrm{H}_{21} \mathrm{~N}_{4}[\mathrm{M}]^{+}$requires 209.1761, found 209.1762. 
${ }^{1} \mathrm{H}$ NMR $\left(400 \mathrm{MHz}, \mathrm{D}_{2} \mathrm{O}\right)$

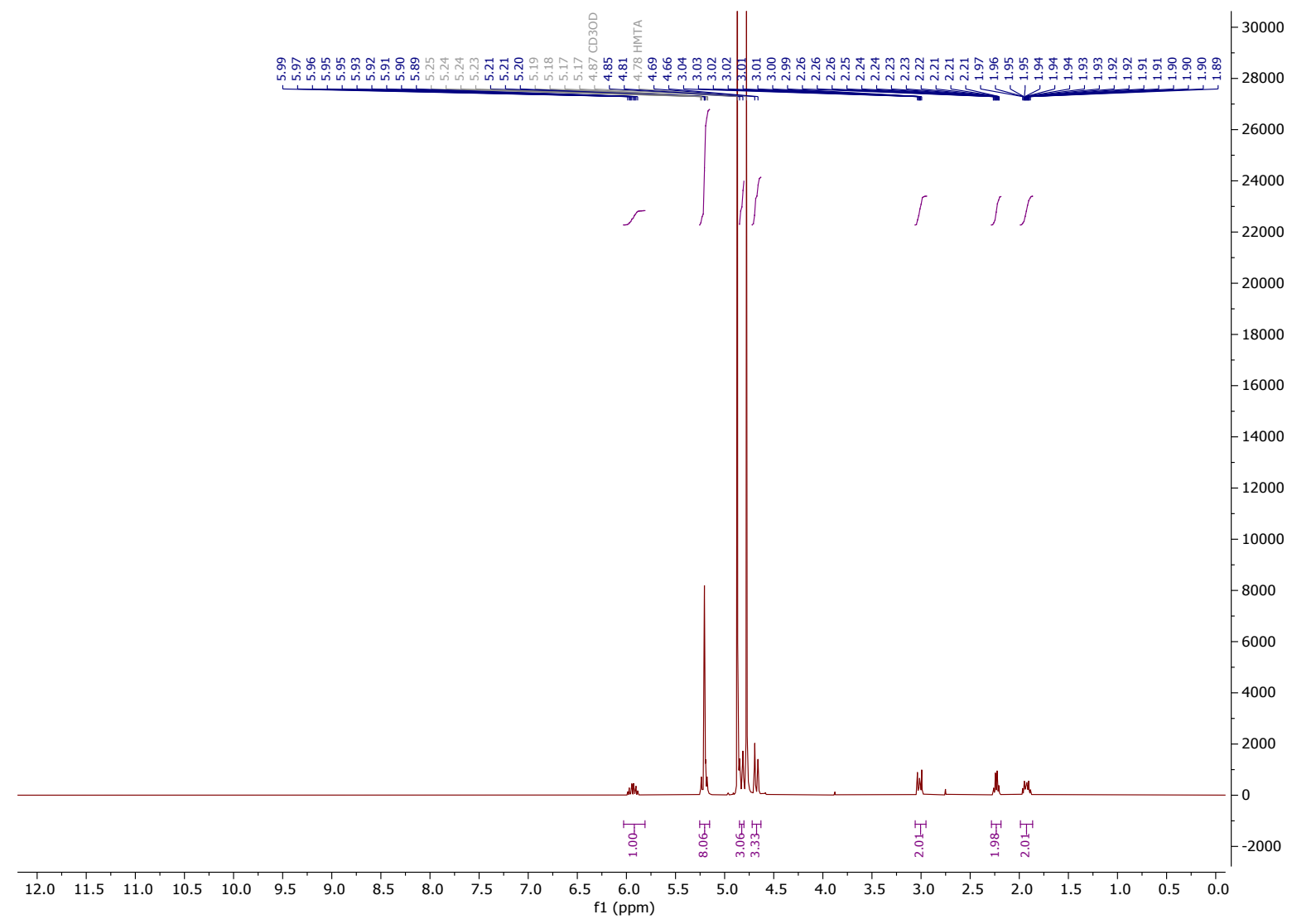

${ }^{13} \mathrm{C}$ NMR $\left(101 \mathrm{MHz}, \mathrm{D}_{2} \mathrm{O}\right)$

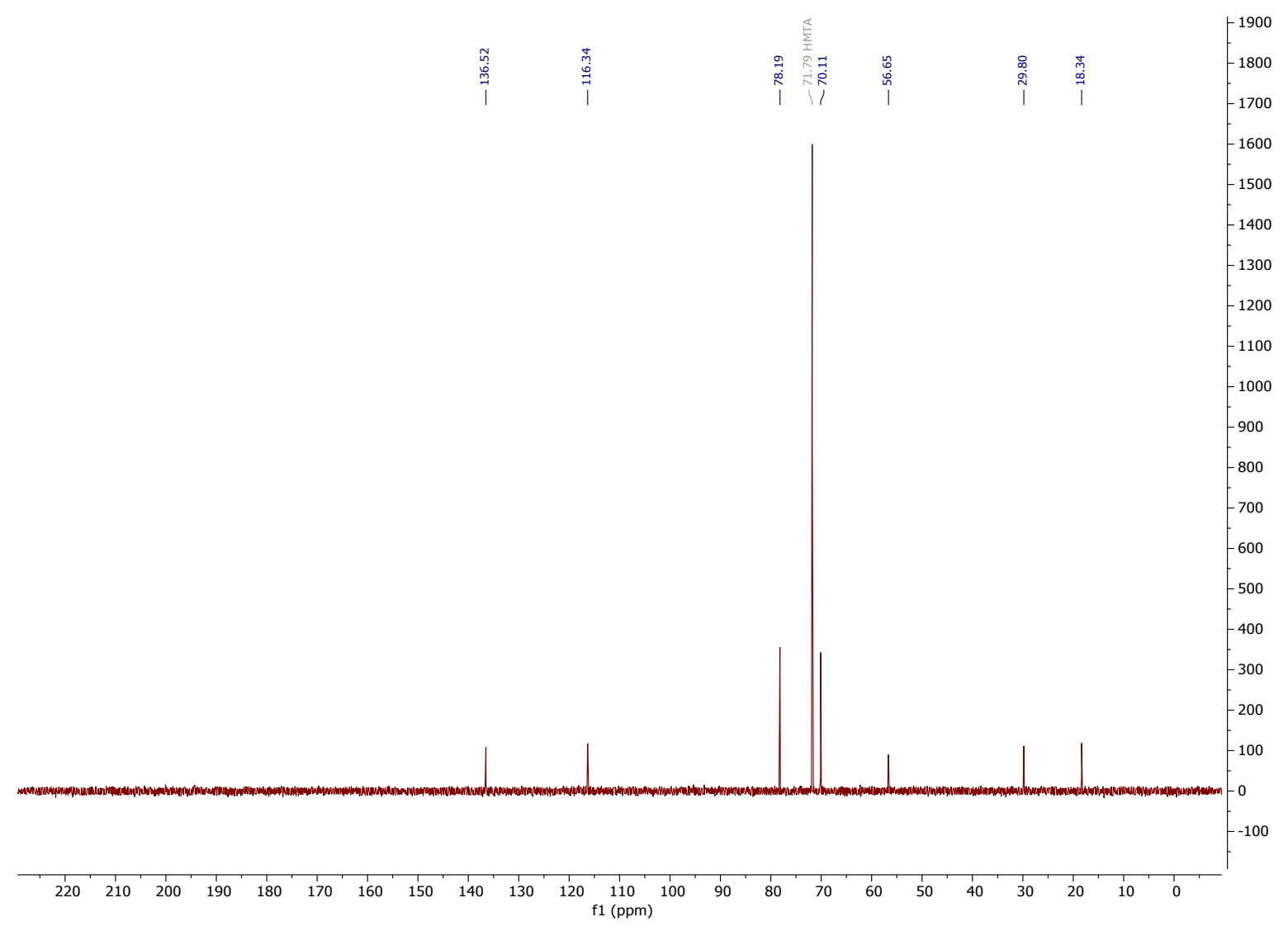


1-(2-Methoxy-2-oxoethyl)-1,3,5,7-tetraazaadamantan-1-ium bromide 9

$\mathrm{Br}^{-}$<smiles>COC(=O)C[N+]12CN3CN(CN(C3)C1)C2</smiles>

1-(2-Methoxy-2-oxoethyl)-1,3,5,7-tetraazaadamantan-1-ium bromide was synthesised according to General Procedure 2 using hexamethylenetetramine (700 mg, $5.0 \mathrm{mmol})$ and 2-methylbromoacetate $(473 \mu \mathrm{L}, 5.0 \mathrm{mmol})$. Yield: $99 \%(1.49 \mathrm{~g}, 5.0 \mathrm{mmol})$.

Chemical Formula: $\mathrm{C}_{9} \mathrm{H}_{17} \mathrm{BrN}_{4} \mathrm{O}_{2}$

Molecular Weight: $293.17 \mathrm{gmol}^{-1}$

Appearance: White solid

$\mathrm{T}_{\mathrm{m}}{ }^{\circ} \mathrm{C}: 158^{\circ} \mathrm{C}$

${ }^{1} \mathrm{H}$ NMR $\left(500 \mathrm{MHz}, \mathrm{DMSO}-d_{6}\right) \delta=5.31(\mathrm{~s}, 6 \mathrm{H}), 4.65(\mathrm{~d}, J=12.7 \mathrm{~Hz}, 3 \mathrm{H}), 4.52(\mathrm{~d}, J=12.7$ $\mathrm{Hz}, 3 \mathrm{H}), 3.91(\mathrm{~s}, 2 \mathrm{H}), 3.77(\mathrm{~s}, 3 \mathrm{H})$.

${ }^{13} \mathrm{C}$ NMR $\left(101 \mathrm{MHz}, \mathrm{DMSO}-d_{6}\right) \delta=164.4,79.1,70.0,54.4,52.9$.

FT-ATR $v_{\max } 3500,3437,2952,1743,1460,1380,1248,1215,1146,1039,1000,947$, $810,699,647,585,494,446$

HRMS $m / z$ (ESI) ${ }^{+}$calcd. for $\mathrm{C}_{9} \mathrm{H}_{18} \mathrm{~N}_{4} \mathrm{O}_{2}[\mathrm{M}]^{+}$required 213.1346, found 213.1343 .

DSC determined melting point and decomposition is in agreement with the literature. ${ }^{14}$ 
${ }^{1} \mathrm{H}$ NMR $\left(500 \mathrm{MHz}, \mathrm{DMSO}-d_{6}\right)$

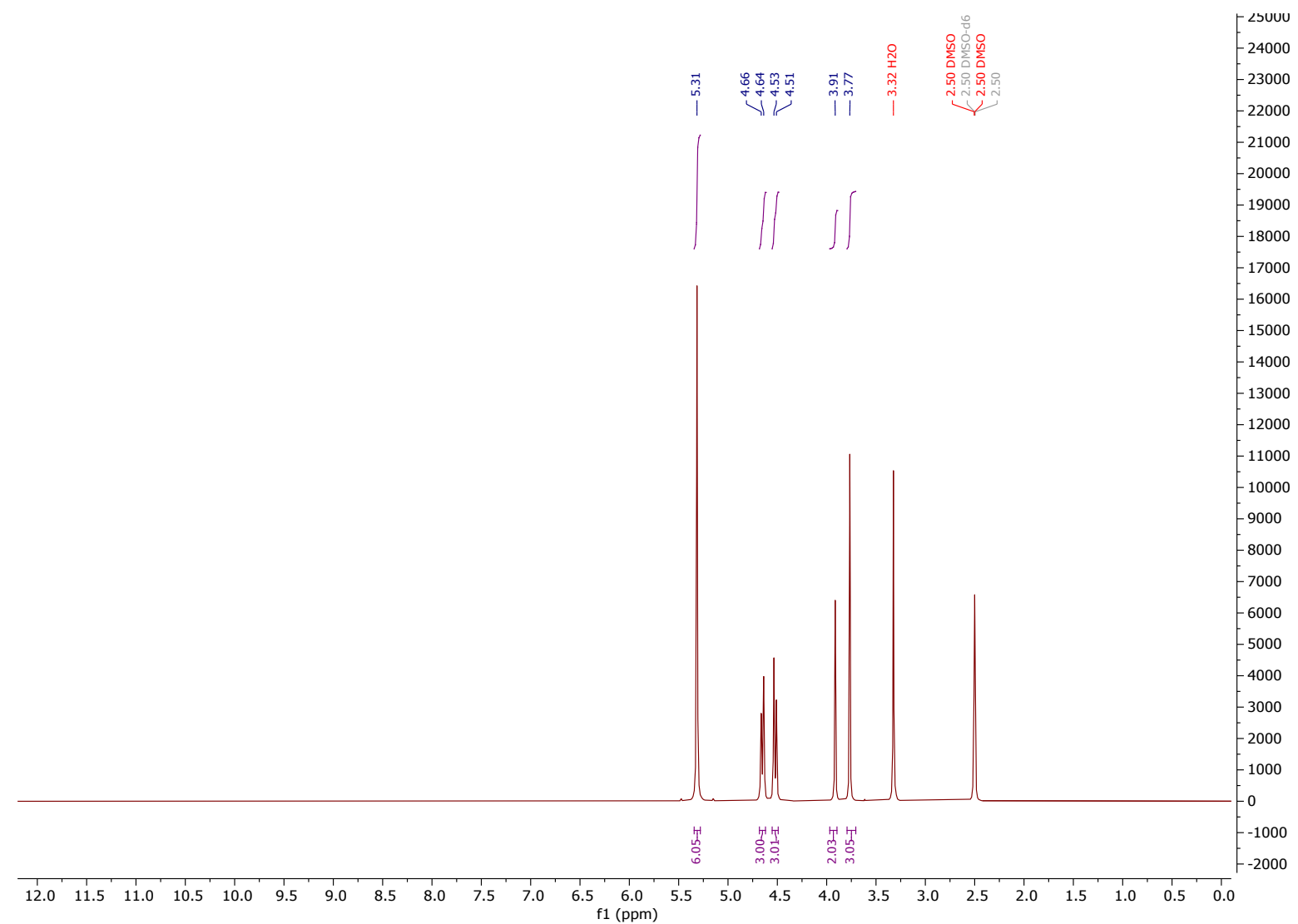

${ }^{13} \mathrm{C}$ NMR (126 MHz, DMSO- $d_{6}$ )

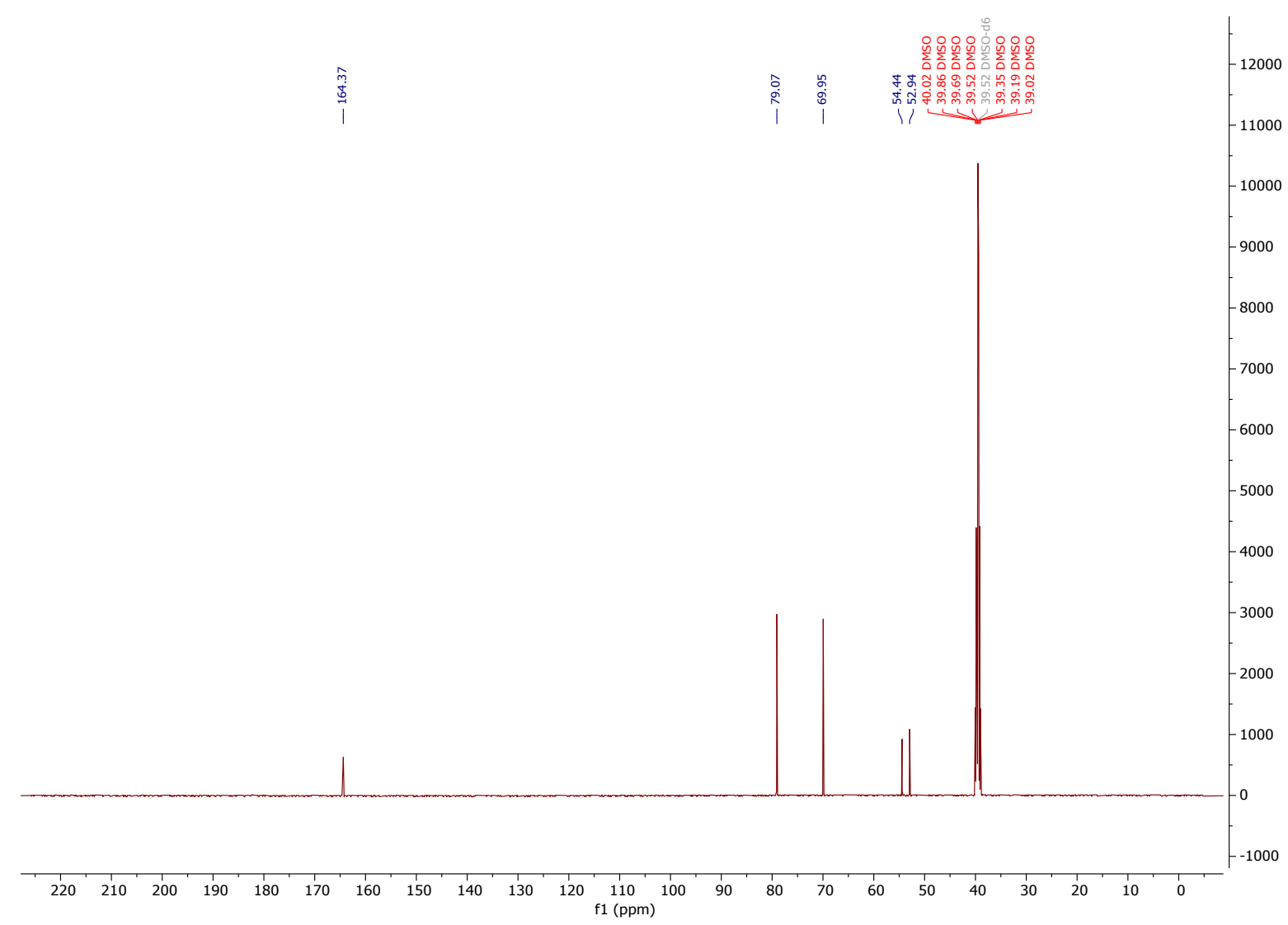


(E)-1-(4-Methoxy-4-oxobut-2-en-1-yl)-1,3,5,7-tetraazaadamantan-1-ium bromide 10<smiles>COC(=O)/C=C/C[N+]12CN3CN(CN(C3)C1Br)C2</smiles>

(E)-1-(4-Methoxy-4-oxobut-2-en-1-yl)-1,3,5,7-tetraazaadamantan-1-ium bromide was synthesised according to General Procedure 2 using hexamethylenetetramine $(701 \mathrm{mg}$, $5.0 \mathrm{mmol})$ and methyl 4-bromocrotonate $(0.895 \mathrm{~g}, 5.0 \mathrm{mmol})$. Crude mass recovery after filtration: $1.04 \mathrm{~g}$. Yield: $90 \%$ (862 $\mathrm{mg}, 2.47 \mathrm{mmol})$.

Chemical Formula: $\mathrm{C}_{11} \mathrm{H}_{19} \mathrm{BrN}_{4} \mathrm{O}_{2}$

Molecular Weight: $319.20 \mathrm{gmol}^{-1}$

Appearance: White solid

${ }^{1} \mathrm{H}$ NMR $\left(400 \mathrm{MHz}, \mathrm{D}_{2} \mathrm{O}\right) \delta=6.99(\mathrm{dt}, \mathrm{J}=15.5,7.7 \mathrm{~Hz}, 1 \mathrm{H}), 6.45(\mathrm{dt}, J=15.5,1.2 \mathrm{~Hz}$, $1 \mathrm{H}), 5.21(\mathrm{~s}, 6 \mathrm{H}), 4.78-4.73(\mathrm{~m}, 3 \mathrm{H}), 4.66-4.57(\mathrm{~m}, 3 \mathrm{H}), 3.84(\mathrm{~s}, 3 \mathrm{H}), 3.79(\mathrm{dd}, \mathrm{J}=$ $7.8,1.2 \mathrm{~Hz}, 2 \mathrm{H})$.

${ }^{13} \mathrm{C}$ NMR $\left(101 \mathrm{MHz}, \mathrm{D}_{2} \mathrm{O}\right) \delta=166.8,132.2,130.6,78.6,70.0,56.6,52.6$.

FT-ATR $v_{\max } 2970,1730,1660,1464,1436,1400,1376,1355,1331,1319,1271,1238$, 1213, 1184, 1137, 1104, 1051, 998, 959, 908, 857, 820, 782, 732, 703, 671, 652, 495.

HRMS $m / z$ (ESI) ${ }^{+}$calcd. for $\mathrm{C}_{11} \mathrm{H}_{19} \mathrm{~N}_{4} \mathrm{O}_{2}[\mathrm{M}]^{+}$requires 239.1503 found 239.1505 . 
${ }^{1} \mathrm{H}$ NMR $\left(400 \mathrm{MHz}, \mathrm{D}_{2} \mathrm{O}\right)$

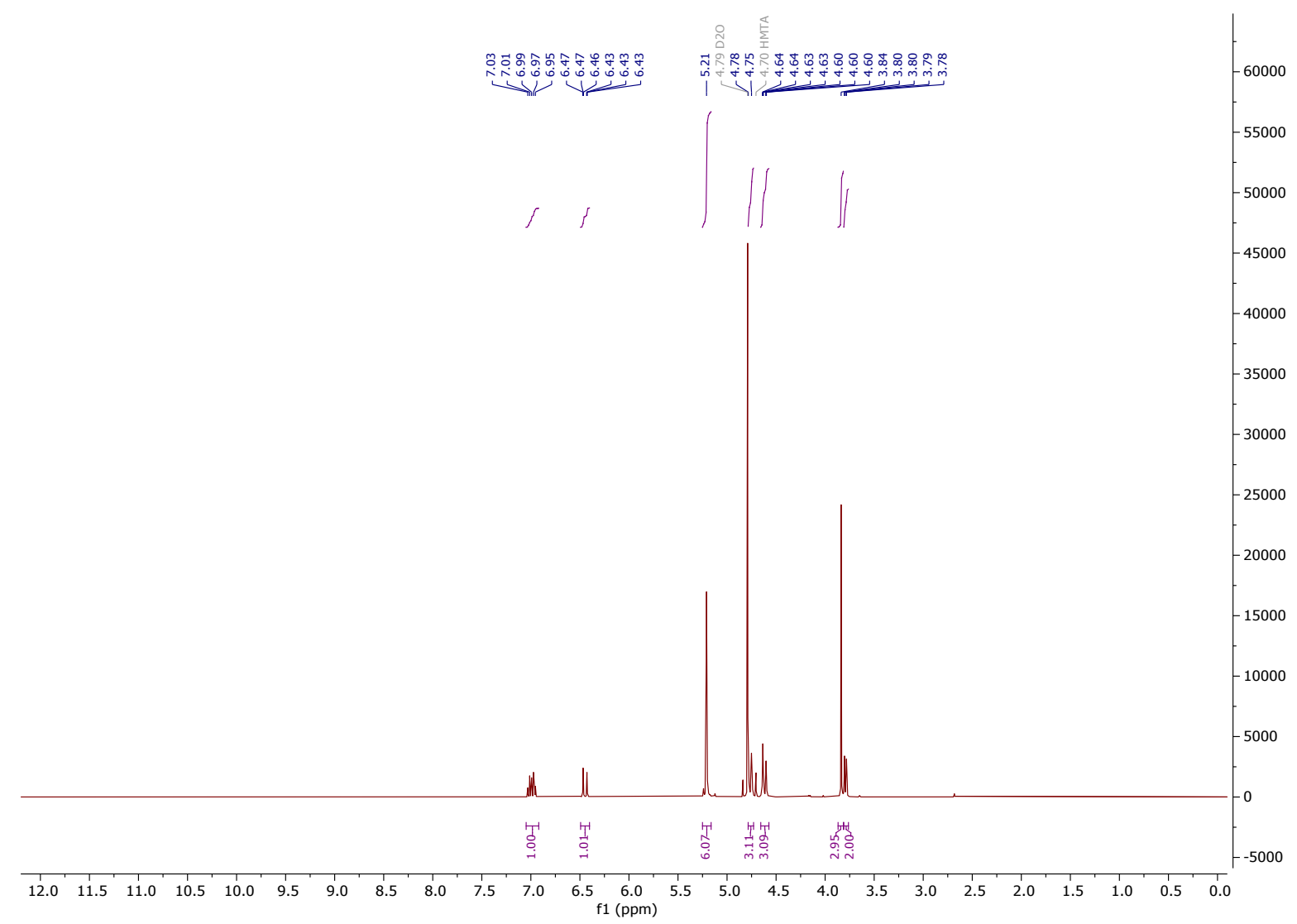

${ }^{13} \mathrm{C}$ NMR $\left(101 \mathrm{MHz}, \mathrm{D}_{2} \mathrm{O}\right)$

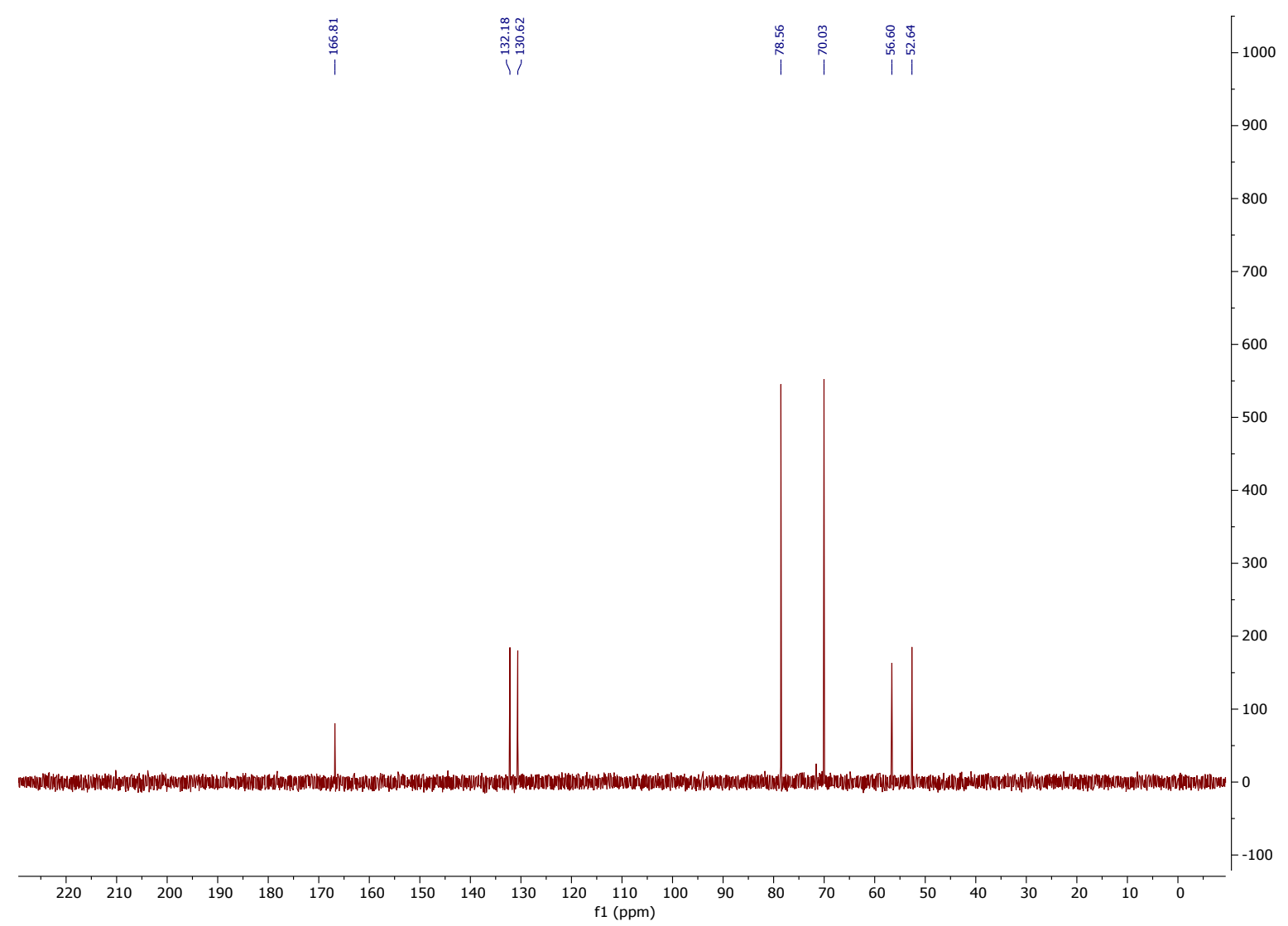


<smiles>CCC#CC[N+]12CN3CN(CN(C3)C1)C2</smiles>

1-(Pent-2-yn-1-yl)-1,3,5,7-tetraazaadamantan-1-ium bromide was synthesised according to General Procedure 2 using hexamethylenetetramine $(701 \mathrm{mg}, 5.0 \mathrm{mmol})$ and 1-bromo2-pentyne (735 mg, $5.0 \mathrm{mmol})$. Yield: $82 \%(1.18 \mathrm{~g}, 4.10 \mathrm{mmol})$.

Chemical Formula: $\mathrm{C}_{11} \mathrm{H}_{19} \mathrm{BrN}_{4}$

Molecular Weight: $287.205 \mathrm{gmol}^{-1}$

Appearance: Pale yellow solid

${ }^{1} \mathrm{H}$ NMR $\left(500 \mathrm{MHz}, \mathrm{DMSO}-d_{6}\right) \delta=5.11(\mathrm{~s}, 6 \mathrm{H}), 4.64(\mathrm{~d}, \mathrm{~J}=12.5 \mathrm{~Hz}, 3 \mathrm{H}), 4.47(\mathrm{~d}, J=$ $12.8 \mathrm{~Hz}, 3 \mathrm{H}), 3.81(\mathrm{t}, \mathrm{J}=2.4 \mathrm{~Hz}, 2 \mathrm{H}), 2.33(\mathrm{qt}, \mathrm{J}=7.4,2.3 \mathrm{~Hz}, 2 \mathrm{H}), 1.14(\mathrm{t}, J=7.5 \mathrm{HZ}$, $3 \mathrm{H})$.

${ }^{13} \mathrm{C}$ NMR $\left(126 \mathrm{MHz}\right.$, DMSO- $\left.d_{6}\right) \delta=94.2,77.9,69.9,65.2,45.6,13.1,11.9$.

FT-ATR $v_{\max }: 2973,2934,2890,1464,1272,1117,1042,1003,936,813,778,654,498$.

HRMS $m / z$ (ESI) ${ }^{+}$calcd. for $\mathrm{C}_{11} \mathrm{H}_{19} \mathrm{~N}_{4}[\mathrm{M}]^{+}$required 207.1604, found 207.1603 
${ }^{1} \mathrm{H}$ NMR $\left(500 \mathrm{MHz}\right.$, DMSO- $\left.d_{6}\right)$

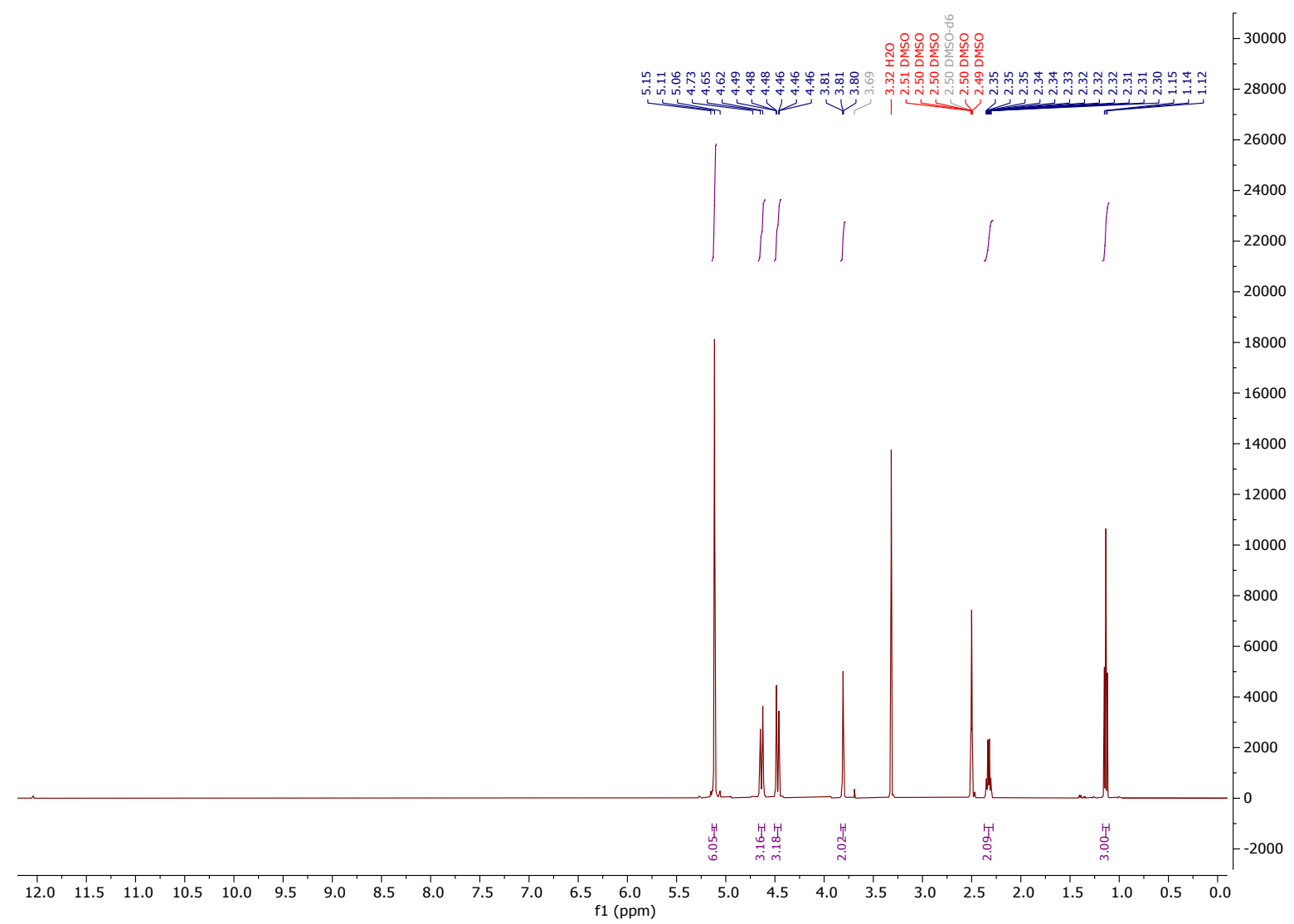

${ }^{13} \mathrm{C}$ NMR (126 MHz, DMSO- $d_{6}$ )

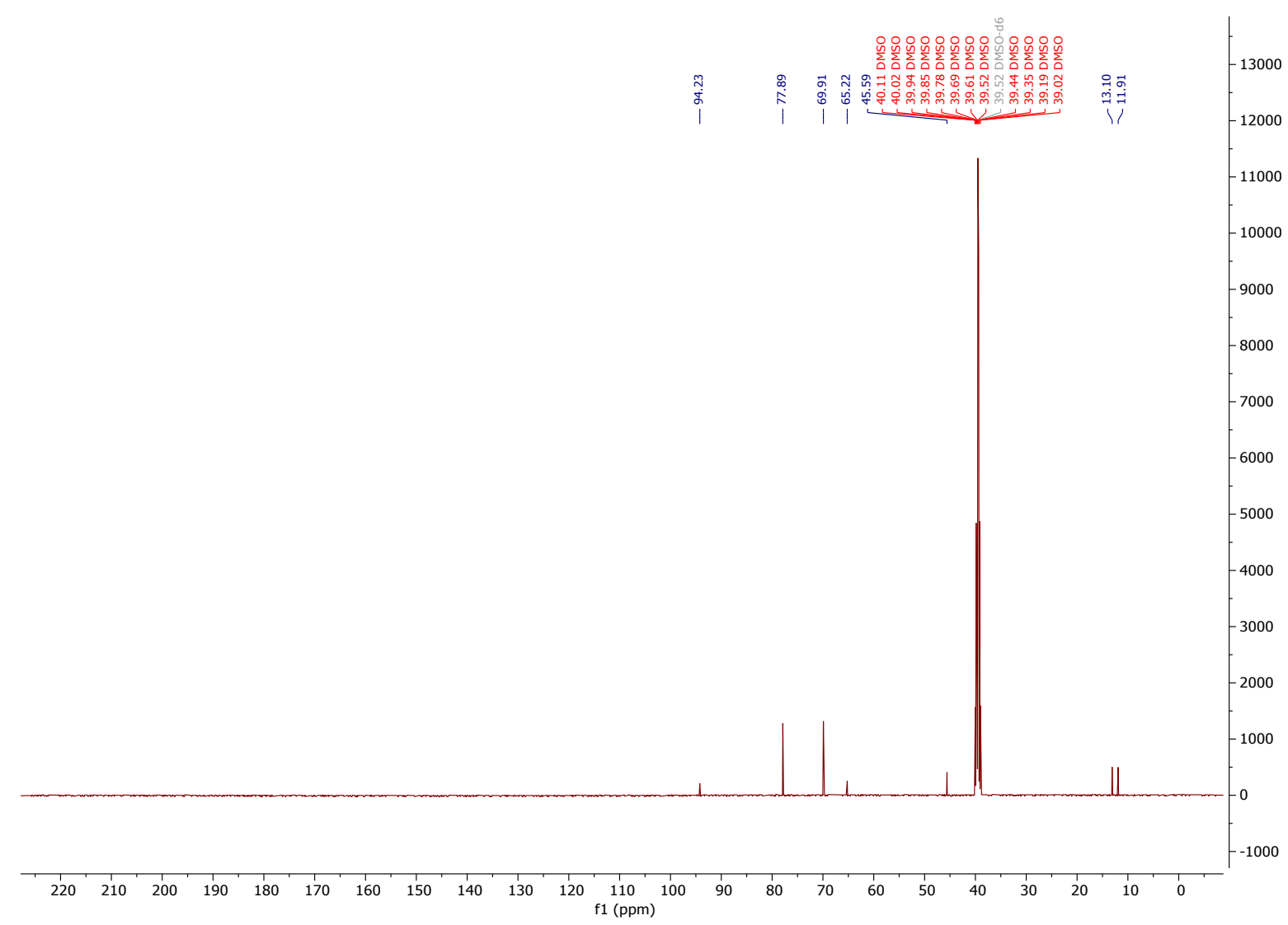


<smiles>C=C(C)C[N+]12CN3CN(CN(C3)C1)C2</smiles>

1-(2-Methylallyl)-1,3,5,7-tetraazaadamantan-1-ium bromide was synthesised according to General Procedure 2 using hexamethylenetetramine $(701 \mathrm{mg}, 5.0 \mathrm{mmol}$ ) and 3-bromo2-methylpropene $(675 \mathrm{mg}, 5.0 \mathrm{mmol})$. Yield: $83 \%(1.14 \mathrm{~g}, 4.12 \mathrm{mmol})$.

Chemical Formula: $\mathrm{C}_{10} \mathrm{H}_{19} \mathrm{BrN}_{4}$

Molecular Weight: $275.194 \mathrm{gmol}^{-1}$

Appearance: White solid

$\mathrm{T}_{\mathrm{m}}{ }^{\circ} \mathrm{C}: 160^{\circ} \mathrm{C}$.

${ }^{1} \mathrm{H}$ NMR $\left(500 \mathrm{MHz}\right.$, DMSO- $\left.d_{6}\right) \delta=5.45(\mathrm{t}, J=1.6 \mathrm{~Hz}, 1 \mathrm{H}), 5.31(\mathrm{~s}, 1 \mathrm{H}), 5.15(\mathrm{~s}, 6 \mathrm{H}), 4.61$ $(\mathrm{d}, J=12.6 \mathrm{~Hz}, 3 \mathrm{H}), 4.50(\mathrm{~d}, J=11.6 \mathrm{~Hz}, 3 \mathrm{H}), 3.47(\mathrm{~s}, 2 \mathrm{H}), 1.92(\mathrm{~s}, 3 \mathrm{H})$.

${ }^{13} \mathrm{C}$ NMR $\left(126 \mathrm{MHz}, \mathrm{DMSO}-d_{6}\right) \delta=132.4,126.3,78.5,70.2,61.7,24.3$.

FT-ATR $v_{\max }$ 2937, 1460, 1266, 1221, 1111, 1015, 955, 929, 814, 795, 649, 499.

HRMS $m / z(E S I)^{+}$calcd. for $\mathrm{C}_{10} \mathrm{H}_{19} \mathrm{~N}_{4}[\mathrm{M}]^{+}$required 195.1604 , found 195.1602 .

DSC determined melting point followed by decomposition were in agreement with the literature. ${ }^{1}$ 
${ }^{1} \mathrm{H}$ NMR $\left(500 \mathrm{MHz}\right.$, DMSO- $\left.d_{6}\right)$

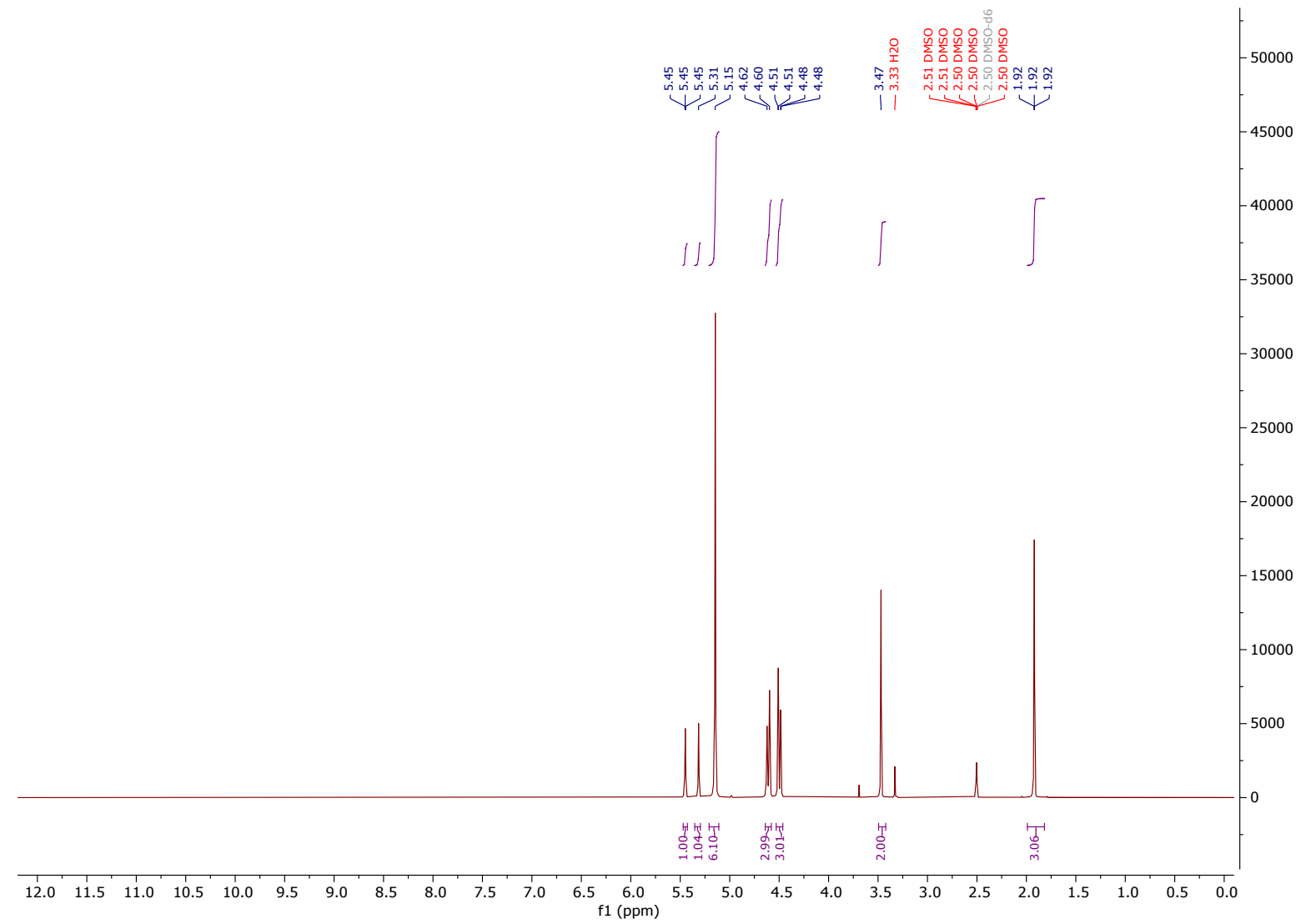

${ }^{13} \mathrm{C}$ NMR (126 MHz, DMSO- $\left.d_{6}\right)$

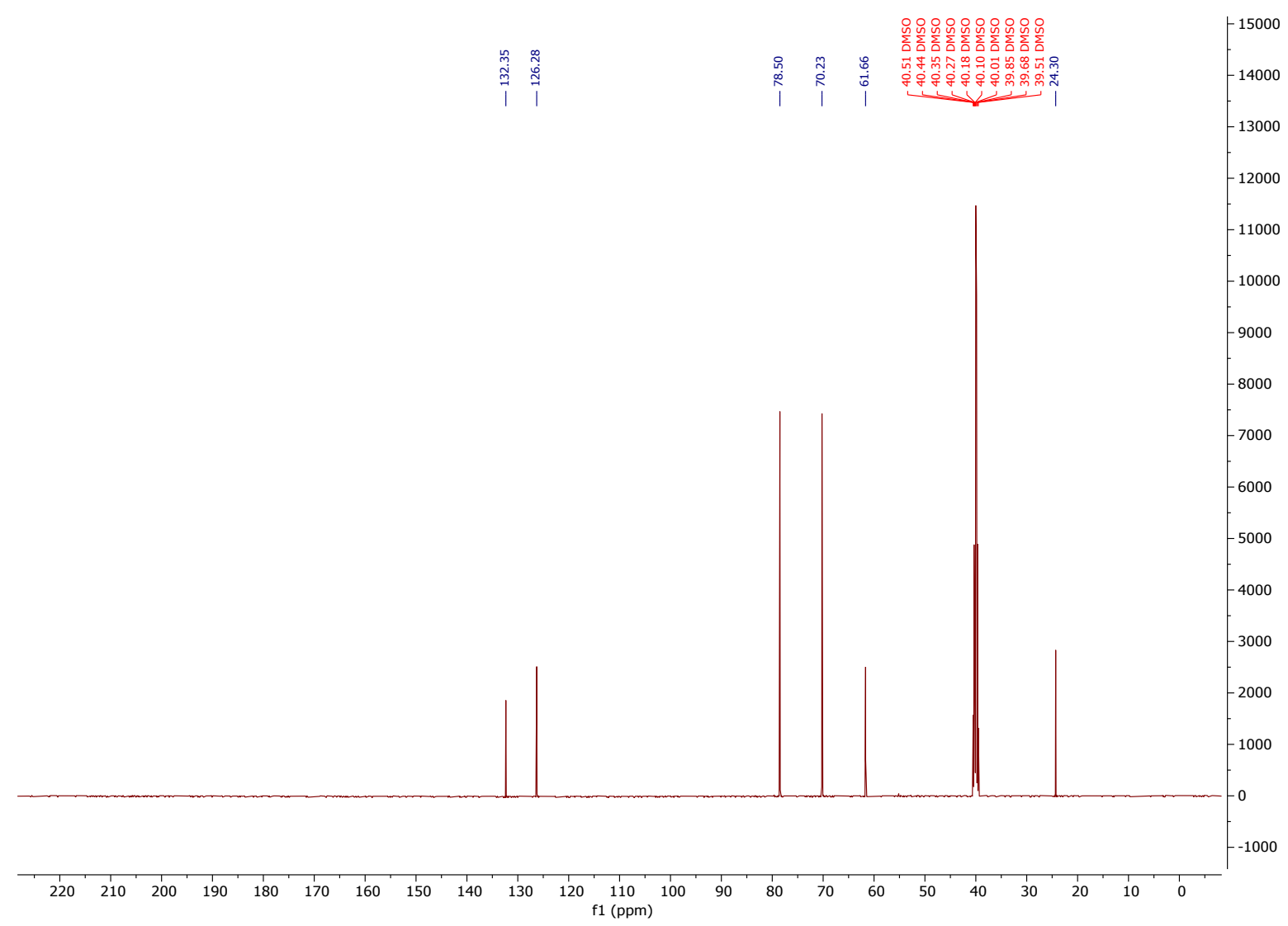


(Z)-1-(4-Chlorobut-2-en-1-yl)-1,3,5,7-tetraazaadamantan-1-ium chloride $\mathbf{1 3}$<smiles>ClC/C=C\C[N+]12CN3CN(CN(C3)C1)C2</smiles>

(Z)-1-(4-Chlorobut-2-en-1-yl)-1,3,5,7-tetraazaadamantan-1-ium chloride was synthesised according to General Procedure 2 using hexamethylenetetramine $(5.61 \mathrm{~g}$, $40.0 \mathrm{mmol}$ ) and cis-1,4-dichlorobutene (4.21 mL, $40.0 \mathrm{mmol})$. Yield: 99\% (10.50 g, 39.6 $\mathrm{mmol}$ ).

Chemical Formula: $\mathrm{C}_{10} \mathrm{H}_{18} \mathrm{Cl}_{2} \mathrm{~N}_{4}$

Molecular Weight: $265.182 \mathrm{gmol}^{-1}$

Appearance: White solid

${ }^{1} \mathrm{H}$ NMR $\left(500 \mathrm{MHz}, \mathrm{DMSO}-d_{6}\right) \delta=6.24-6.14(\mathrm{~m}, 1 \mathrm{H}), 5.90-5.81(\mathrm{~m}, 1 \mathrm{H}), 5.16(\mathrm{~s}, 6 \mathrm{H})$, $4.61(\mathrm{~d}, J=12.5 \mathrm{~Hz}, 3 \mathrm{H}), 4.47(\mathrm{~d}, J=12.4 \mathrm{~Hz}, 3 \mathrm{H}), 4.43(\mathrm{~d}, J=8.1 \mathrm{~Hz}, 3 \mathrm{H}), 3.72-3.66$ $(\mathrm{m}, 2 \mathrm{H})$.

${ }^{13} \mathrm{C}$ NMR $\left(126 \mathrm{MHz}\right.$, DMSO- $\left.d_{6}\right) \delta=136.2,118.8,77.5,69.9,51.2,38.7$.

FT-ATR $v_{\max }: 2973,1467,1216,1227,1115,1037,1000,935,822,776,697,651,497$.

HRMS $\mathrm{m} / \mathrm{z}(\mathrm{ESI})^{+}$calcd. for $\mathrm{C}_{10} \mathrm{H}_{18} \mathrm{CIN}_{4}[\mathrm{M}]^{+}$required 229.1215 , found 229.1215 .

${ }^{1} \mathrm{H}$ and ${ }^{13} \mathrm{C}-\mathrm{NMR}$ were in agreement with the literature. ${ }^{15-16}$ 
${ }^{1} \mathrm{H}$ NMR $\left(500 \mathrm{MHz}\right.$, DMSO- $\left.d_{6}\right)$

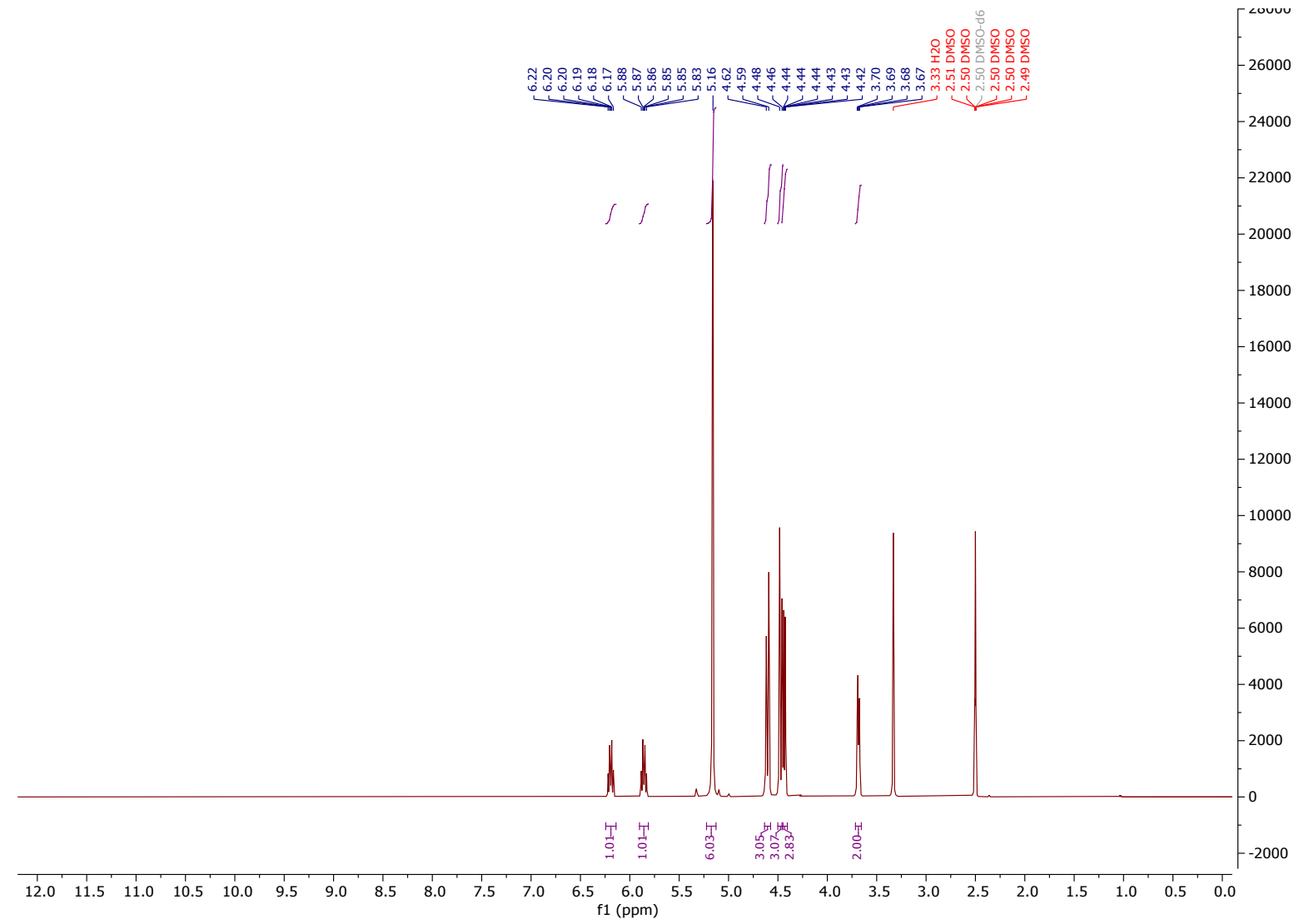

${ }^{13} \mathrm{C}$ NMR (126 MHz, DMSO- $d_{6}$ )

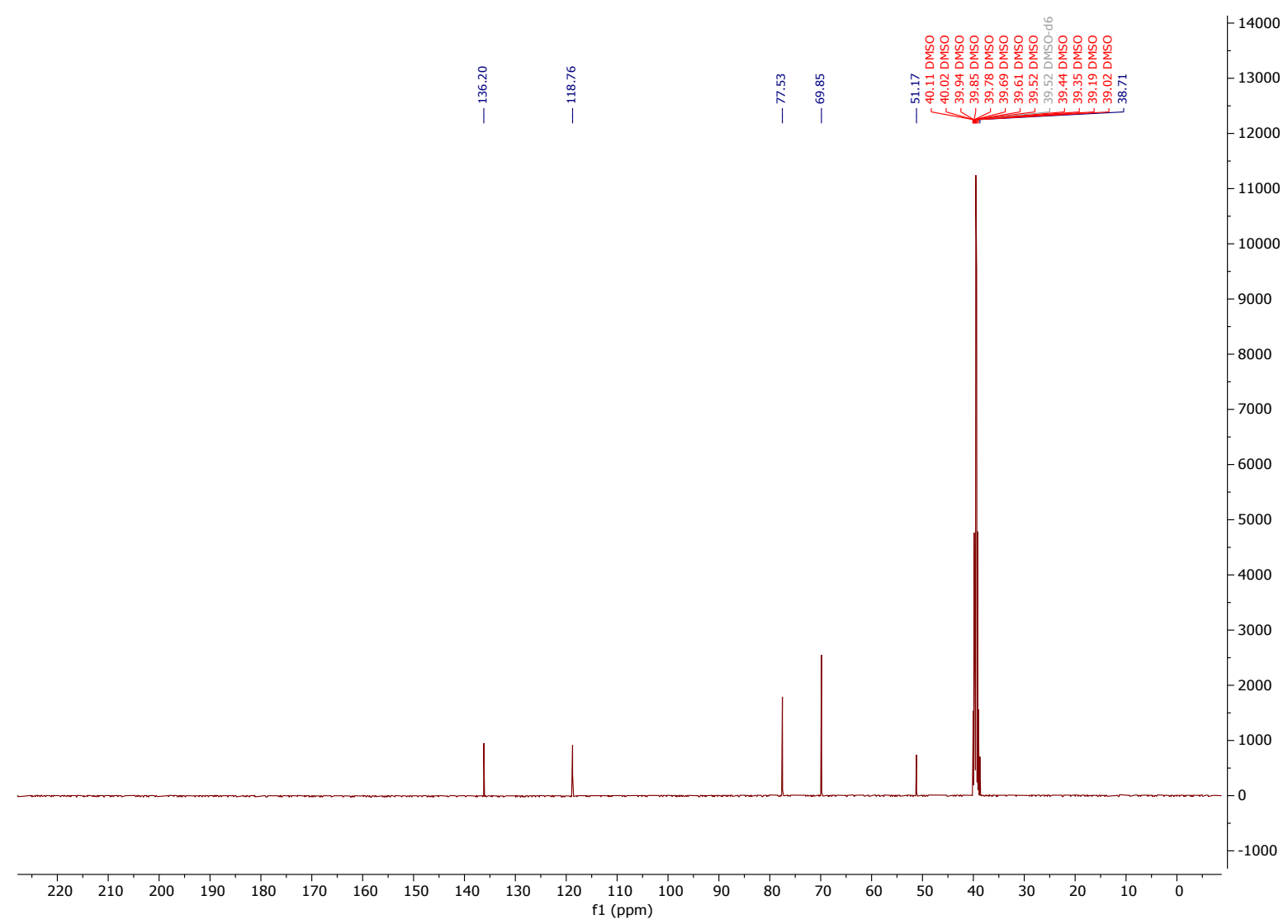


<smiles>C#CCC[N+]12CN3CN(CN(C3)C1)C2</smiles>

1-(But-3-yn-1-yl)-1,3,5,7-tetraazaadamantan-1-ium bromide was synthesised according to General Procedure 2 using hexamethylenetetramine $(701 \mathrm{mg}, 5.0 \mathrm{mmol})$ and 4-bromo1-butyne (665 mg, $5.0 \mathrm{mmol}$ ). Yield: $28 \%$ (382 mg, $1.40 \mathrm{mmol}$ ).

Chemical Formula: $\mathrm{C}_{10} \mathrm{H}_{17} \mathrm{BrN}_{4}$

Molecular Weight: $273.178 \mathrm{gmol}^{-1}$

Appearance: Off-white solid

${ }^{1} \mathrm{H}$ NMR $\left(500 \mathrm{MHz}, \mathrm{DMSO}-d_{6}\right) \delta=5.12(\mathrm{~s}, 6 \mathrm{H}), 4.61(\mathrm{~d}, \mathrm{~J}=12.6 \mathrm{~Hz}, 3 \mathrm{H}), 4.48-4.43(\mathrm{~m}$, $3 \mathrm{H}), 3.15(\mathrm{t}, J=2.6 \mathrm{~Hz}, 1 \mathrm{H}), 2.97(\mathrm{t}, J=7.5 \mathrm{~Hz}, 2 \mathrm{H}), 2.74(\mathrm{td}, J=7.5,2.7 \mathrm{~Hz}, 2 \mathrm{H})$.

${ }^{13} \mathrm{C}$ NMR $\left(126 \mathrm{MHz}, \mathrm{DMSO}-d_{6}\right) \delta=80.2,78.1,74.0,69.80,54.0,10.6$.

FT-ATR $v_{\max }: 3202,2978,2946,1460,1369,1275,1234,1124,998,962,809,707,659$, 501.

HRMS $m / z$ (ESI) ${ }^{+}$calcd. for $\mathrm{C}_{10} \mathrm{H}_{17} \mathrm{~N}_{4}[\mathrm{M}]^{+}$required 193.1448 , found 193.1447 . 
${ }^{1} \mathrm{H}$ NMR $\left(500 \mathrm{MHz}, \mathrm{DMSO}-d_{6}\right)$

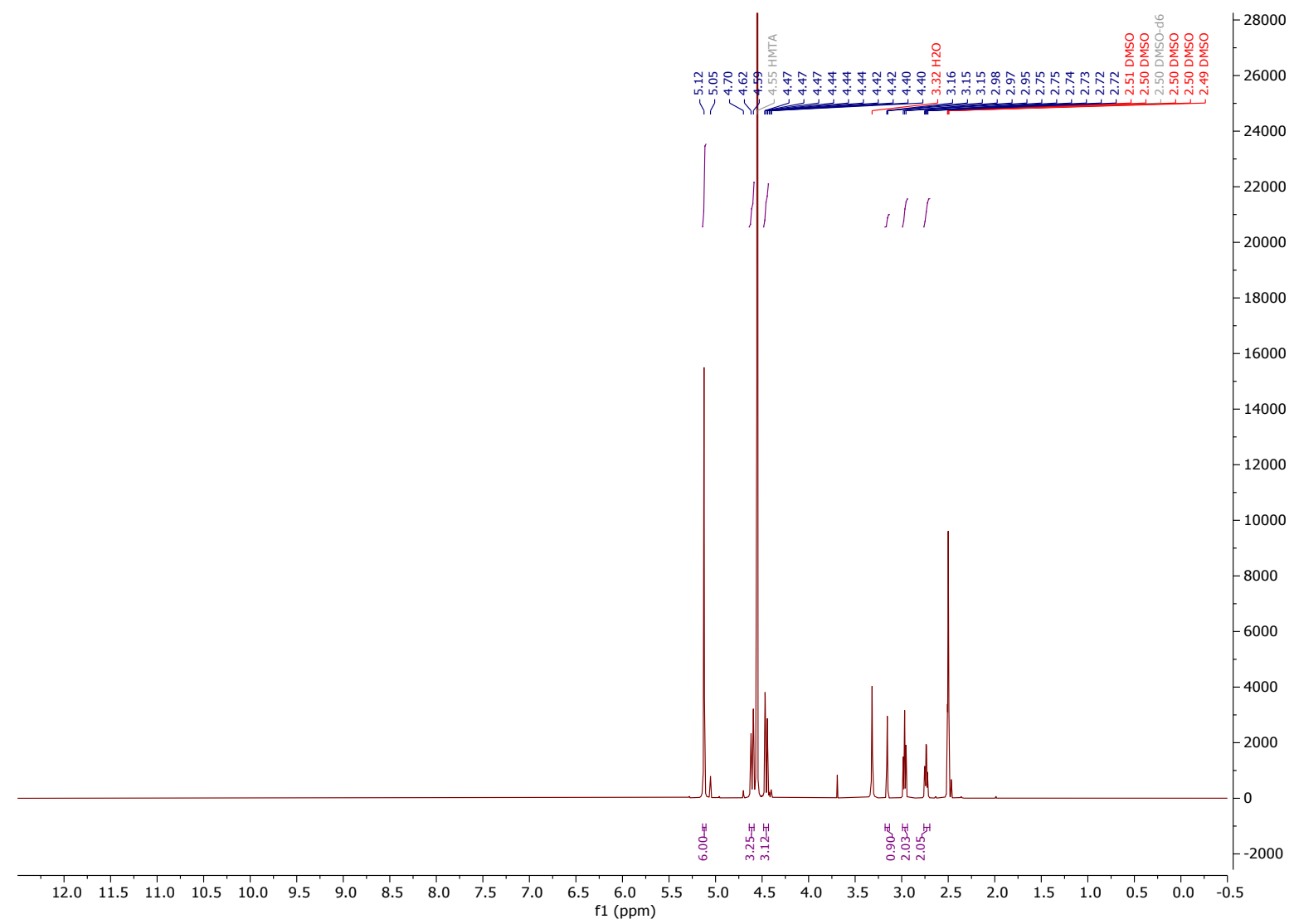

${ }^{13} \mathrm{C}$ NMR (126 MHz, DMSO- $\left.d_{6}\right)$

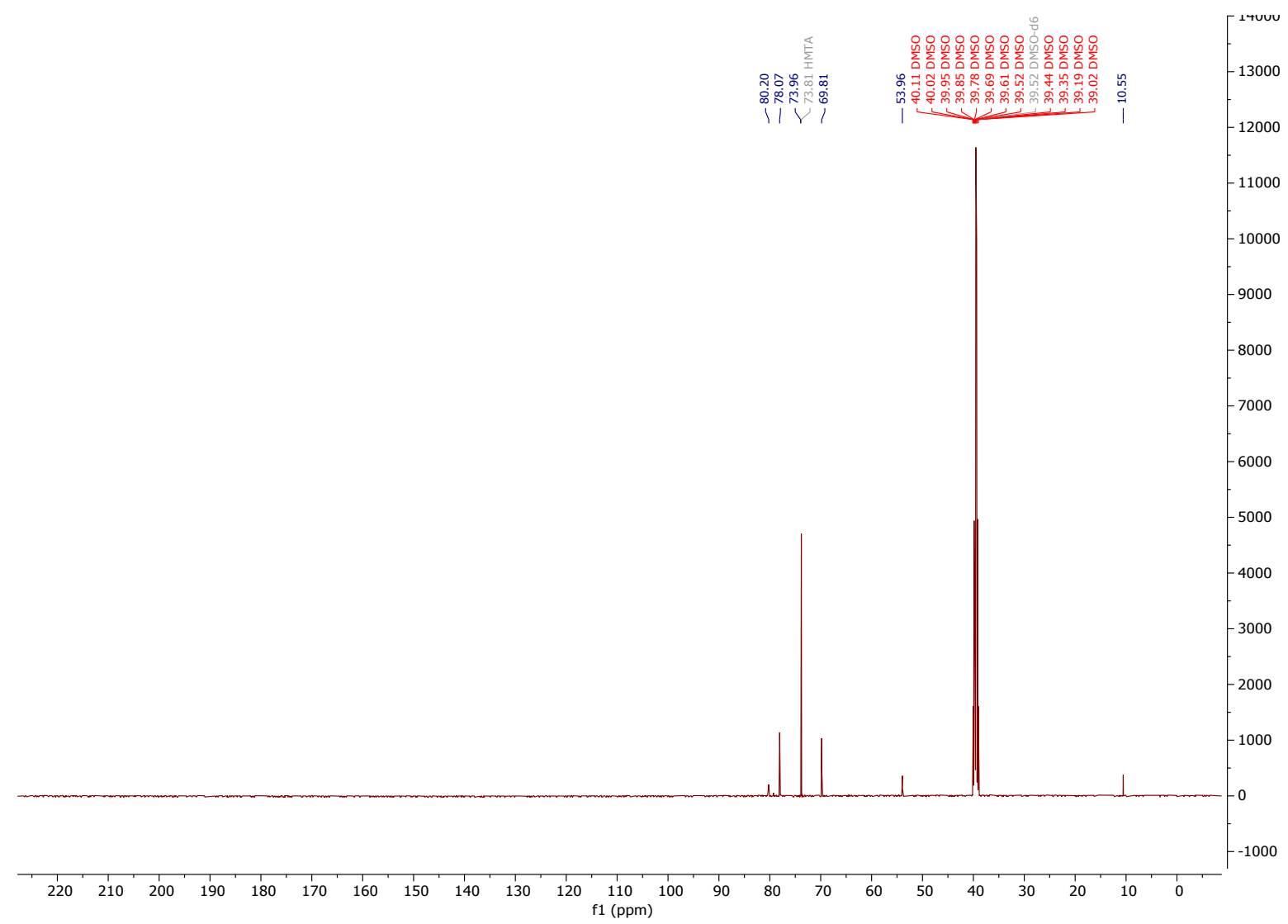


1-(2-Oxo-2-phenylethyl)-1,3,5,7-tetraazaadamantan-1-ium bromide $\mathbf{1 5}$<smiles>O=C(C[N+]12CN3CN(CN(C3)C1)C2)c1ccccc1</smiles>

1-(2-Oxo-2-phenylethyl)-1,3,5,7-tetraazaadamantan-1-ium bromide was synthesised according to General Procedure 2 using hexamethylenetetramine (701 mg, $5.0 \mathrm{mmol}$ ) and 2-bromoacetophenone (995 mg, $5.0 \mathrm{mmol})$. Yield: $97 \%$ (1.65 g, $4.86 \mathrm{mmol})$.

Chemical Formula: $\mathrm{C}_{14} \mathrm{H}_{19} \mathrm{BrN}_{4} \mathrm{O}$

Molecular Weight: $339.237 \mathrm{gmol}^{-1}$

Appearance: White solid

${ }^{1} \mathrm{H}$ NMR $\left(500 \mathrm{MHz}\right.$, DMSO- $\left.d_{6}\right) \delta=8.04-7.96(\mathrm{~m}, 2 \mathrm{H}), 7.81-7.74(\mathrm{~m}, 1 \mathrm{H}), 7.63(\mathrm{t}, \mathrm{J}=$ $7.8 \mathrm{~Hz}, 2 \mathrm{H}), 5.42(\mathrm{~s}, 6 \mathrm{H}), 4.77(\mathrm{~s}, 2 \mathrm{H}), 4.70(\mathrm{~d}, \mathrm{~J}=12.7 \mathrm{~Hz}, 3 \mathrm{H}), 4.64-4.57(\mathrm{~m}, 3 \mathrm{H})$.

${ }^{13} \mathrm{C}$ NMR $\left(126 \mathrm{MHz}, \mathrm{DMSO}-d_{6}\right) \delta=191.3,135.3,134.9,129.5,128.6,79.7,70.7,59.4$.

FT-ATR $v_{\max } 2955,1692,1458,1275,1214,1145,1055,1001,932,825,758,684,498$.

HRMS $m / z(E S I)^{+}$calcd. for $\mathrm{C}_{14} \mathrm{H}_{19} \mathrm{~N}_{4} \mathrm{O}[\mathrm{M}]^{+}$required 259.1553, found 259.1552. 
${ }^{1} \mathrm{H}$ NMR $\left(500 \mathrm{MHz}, \mathrm{DMSO}-d_{6}\right)$

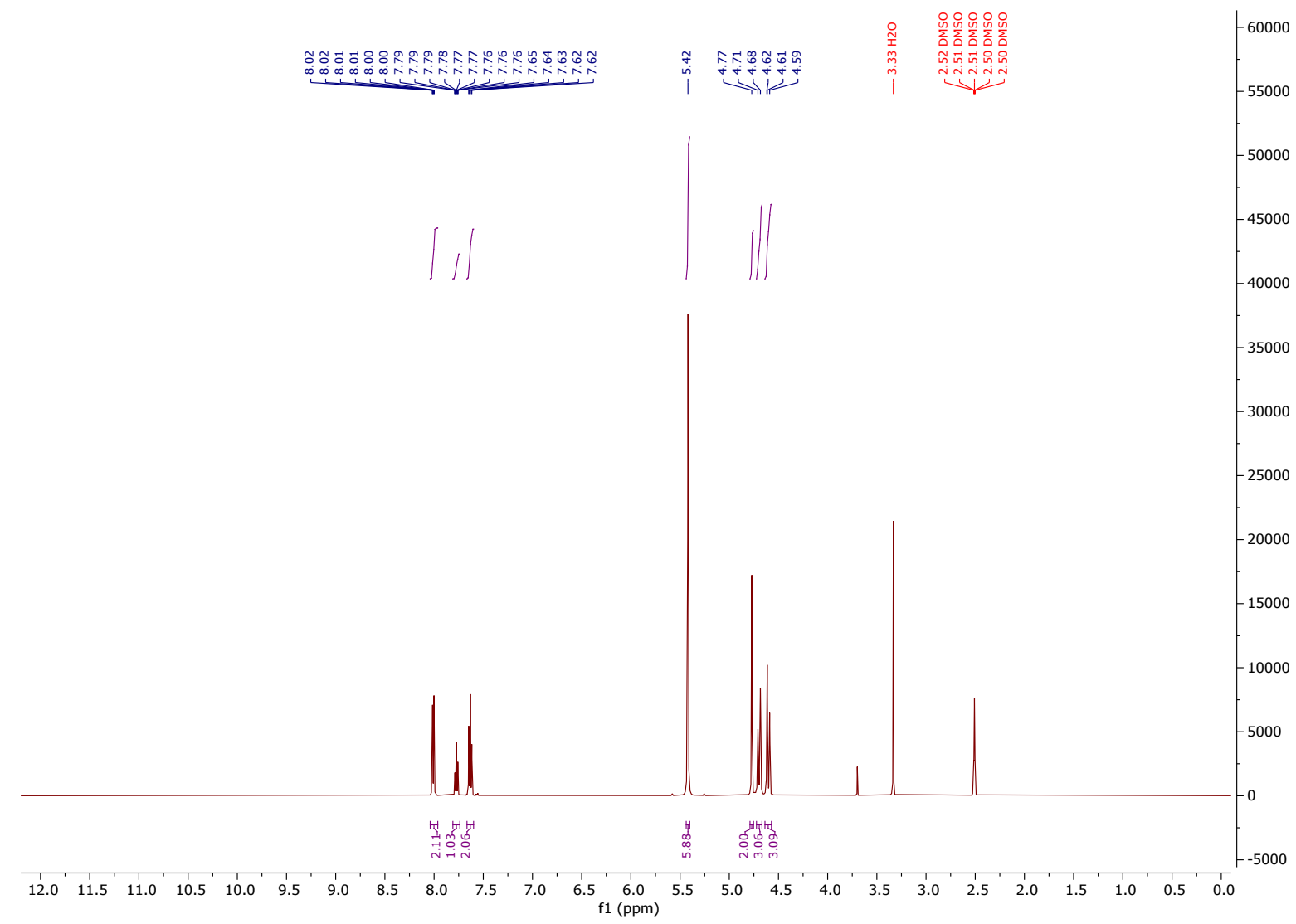

${ }^{13} \mathrm{C}$ NMR (126 MHz, DMSO- $d_{6}$ )

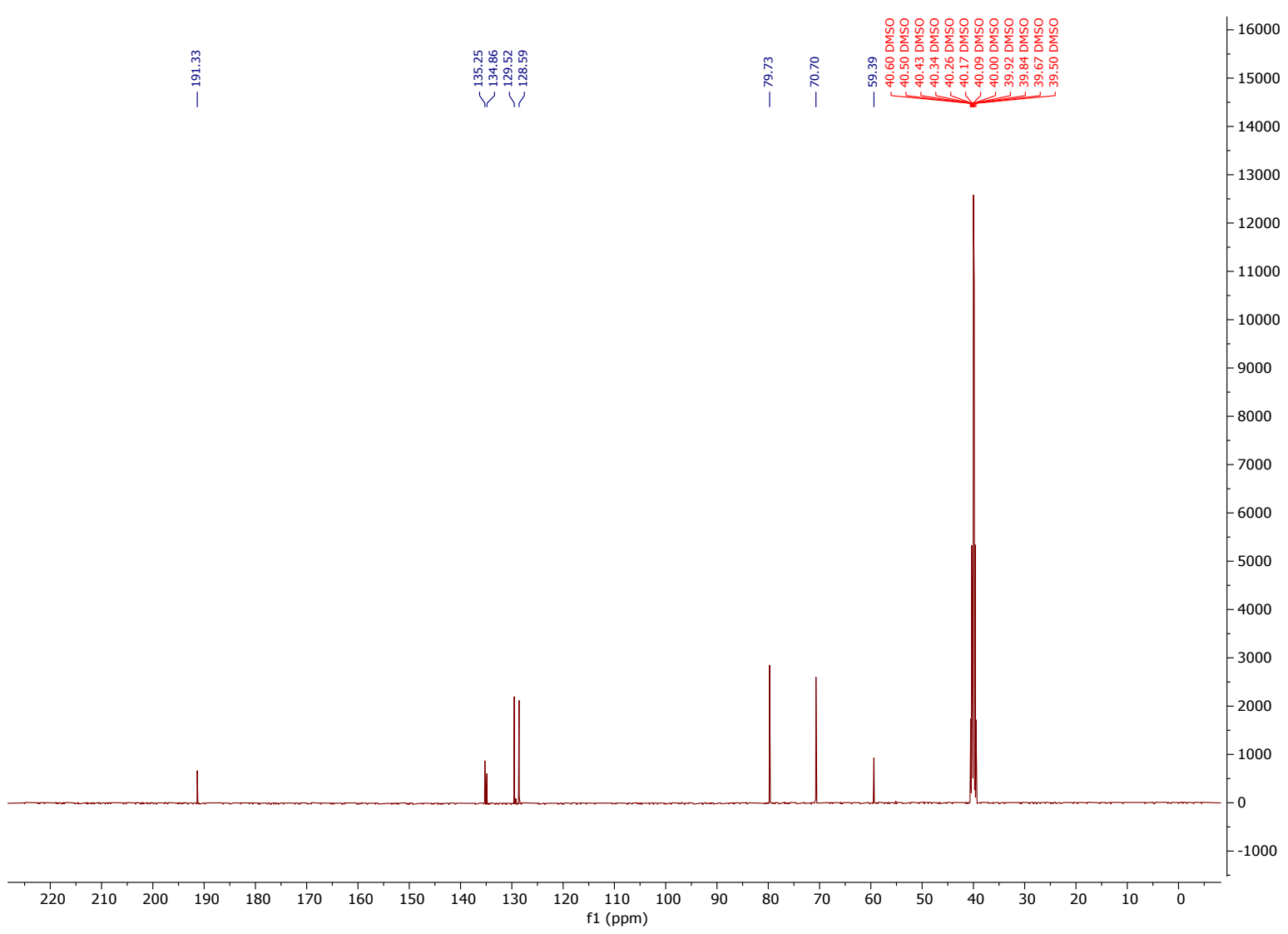


<smiles></smiles>

1-(4-(Methoxycarbonyl)benzyl)-1,3,5,7-tetraazaadamantan-1-ium bromide was synthesised according to General Procedure 2 using hexamethylenetetramine $(701 \mathrm{mg}$, $5.0 \mathrm{mmol})$ and methyl-4-(bromomethyl) benzoate $(1.15 \mathrm{~g}, 5.0 \mathrm{mmol})$. Yield: $97 \%(1.79$ $\mathrm{g}, 4.85 \mathrm{mmol})$.

Chemical Formula: $\mathrm{C}_{15} \mathrm{H}_{21} \mathrm{BrN}_{4} \mathrm{O}_{2}$

Molecular Weight: $369.26 \mathrm{gmol}^{-1}$

Appearance: White solid

$\mathrm{T}_{\mathrm{m}}{ }^{\circ} \mathrm{C}: 177^{\circ} \mathrm{C}$.

${ }^{1} \mathrm{H}$ NMR $\left(400 \mathrm{MHz}, \mathrm{D}_{2} \mathrm{O}\right) \delta=8.20-8.13(\mathrm{~m}, 2 \mathrm{H}), 7.68-7.61(\mathrm{~m}, 2 \mathrm{H}), 5.16(\mathrm{~s}, 6 \mathrm{H}), 4.74$ $-4.69(\mathrm{~m}, 3 \mathrm{H}), 4.55-4.50(\mathrm{~m}, 3 \mathrm{H}), 4.24(\mathrm{~s}, 2 \mathrm{H}), 3.98(\mathrm{~s}, 3 \mathrm{H})$.

${ }^{13} \mathrm{C}$ NMR $\left(101 \mathrm{MHz}, \mathrm{D}_{2} \mathrm{O}\right) \delta=168.4,132.7,131.8,130.2,129.4,78.3,70.0,60.4,52.9$.

FT-ATR $v_{\max } 2951,1715,1430,1372,1316,1273,1222,1184,1108,1043,1001,936$, $891,859,819,769,707,649,614,498,459$

HRMS $m / z$ (ESI) ${ }^{+}$calcd. for $\mathrm{C}_{15} \mathrm{H}_{21} \mathrm{~N}_{4} \mathrm{O}_{2}[M]^{+}$required 289.1659 , found 289.1653 .

DSC determined melting point followed by decomposition were in agreement with the literature. ${ }^{17}$ 
${ }^{1} \mathrm{H}$ NMR $\left(400 \mathrm{MHz}, \mathrm{D}_{2} \mathrm{O}\right)$

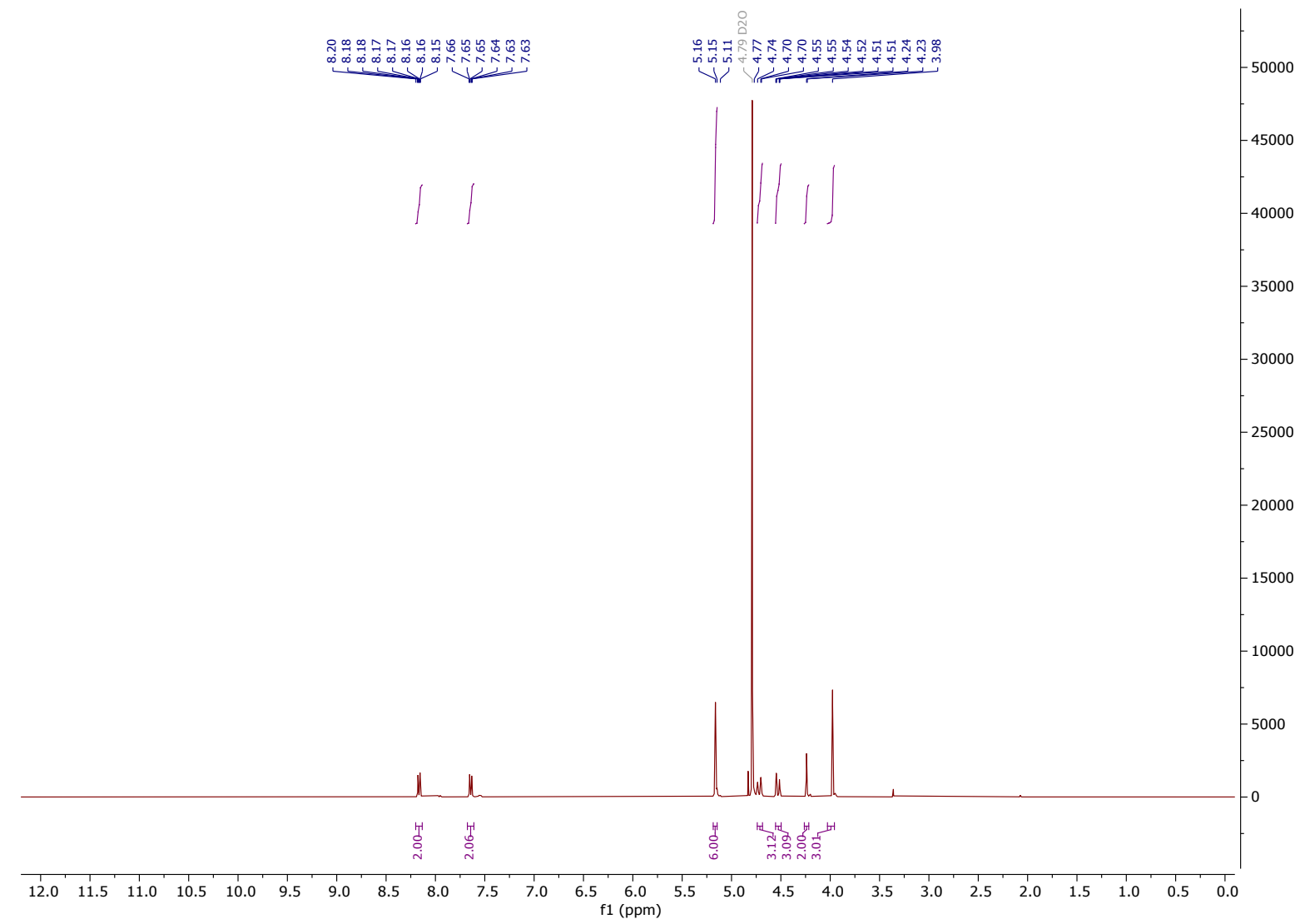

${ }^{13} \mathrm{C}$ NMR $\left(101 \mathrm{MHz}, \mathrm{D}_{2} \mathrm{O}\right)$

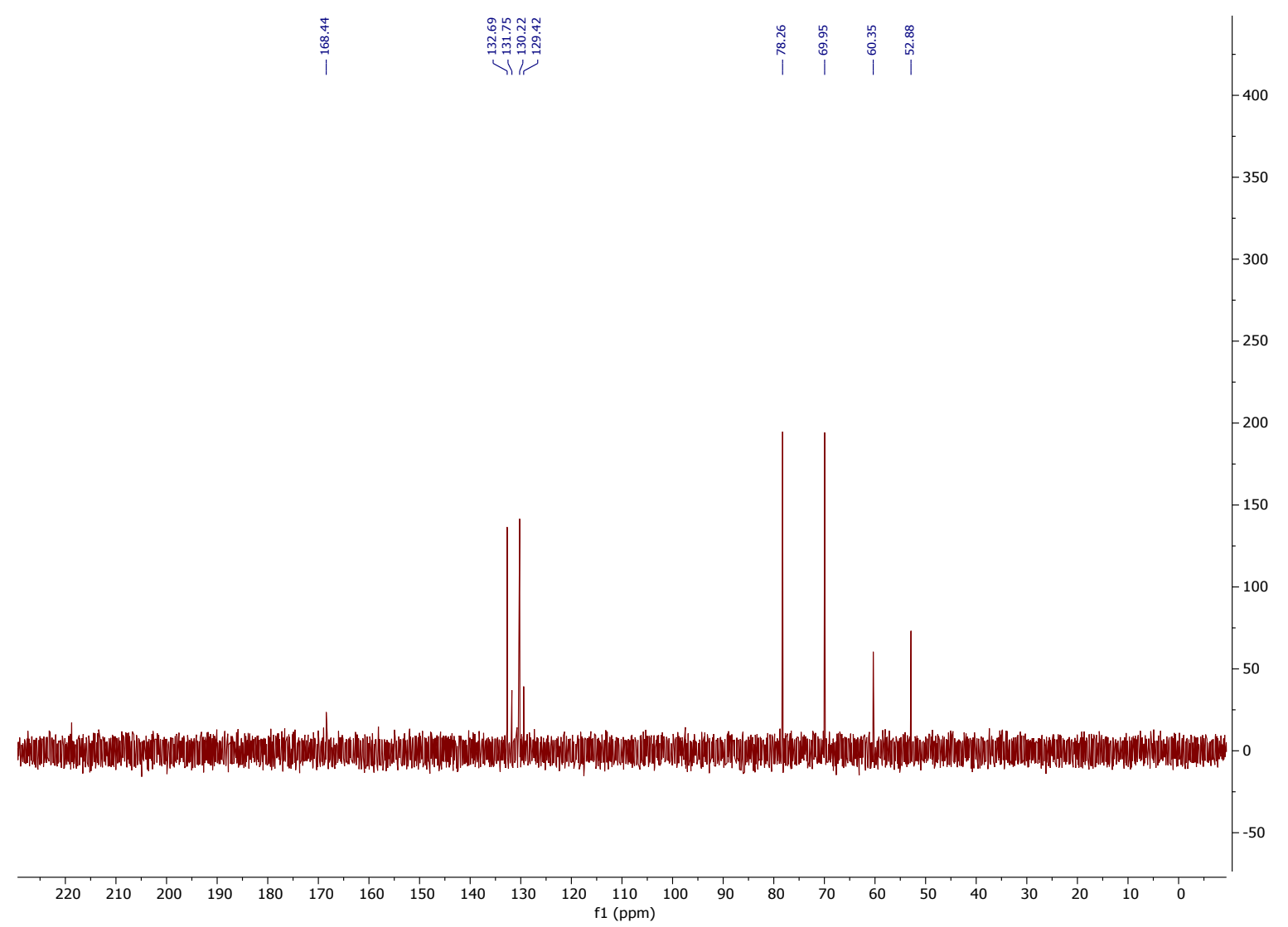


<smiles>COc1ccc(C(=O)C[N+]23CCN(CN(CBr)C2)C3)cc1</smiles>

1-(2-(4-Methoxyphenyl)-2-oxoethyl)-1,3,5,7-tetraazaadamantan-1-ium bromide was synthesised according to General Procedure 2 using hexamethylenetetramine $(701 \mathrm{mg}$, $5.0 \mathrm{mmol})$ and 2-bromo-4'-methoxyacetophenone $(1.15 \mathrm{~g}, 5.0 \mathrm{mmol})$. Yield: $82 \%(1.51$ $\mathrm{g}, 4.08 \mathrm{mmol})$.

Chemical Formula: $\mathrm{C}_{15} \mathrm{H}_{21} \mathrm{BrN}_{4} \mathrm{O}_{2}$

Molecular Weight: $369.263 \mathrm{gmol}^{-1}$

Appearance: White solid

${ }^{1} \mathrm{H}$ NMR $\left(500 \mathrm{MHz}, \mathrm{DMSO}-d_{6}\right) \delta=7.98(\mathrm{~d}, J=8.9 \mathrm{~Hz}, 2 \mathrm{H}), 7.21-7.08(\mathrm{~m}, 2 \mathrm{H}), 5.40(\mathrm{~s}$, $6 \mathrm{H}), 4.72-4.65(\mathrm{~m}, 5 \mathrm{H}), 4.58(\mathrm{~d}, \mathrm{~J}=11.9 \mathrm{~Hz}, 3 \mathrm{H}), 3.88(\mathrm{~s}, 3 \mathrm{H})$.

${ }^{13} \mathrm{C}$ NMR $\left(126 \mathrm{MHz}, \mathrm{DMSO}-d_{6}\right) \delta=189.1,164.4,130.7,127.2,114.3,79.2,70.2,58.5$, 55.8 .

FT-ATR $v_{\max }: 2973,2899,1683,1604,1460,1418,1247,1172,1138,1041,1002,934$, $824,787,645,592,494$.

HRMS $m / z$ (ESI) + calcd. for $\mathrm{C}_{15} \mathrm{H}_{21} \mathrm{~N}_{4} \mathrm{O}_{2}[\mathrm{M}]^{+}$required 289.1659, found 289.1661. 
${ }^{1} \mathrm{H}$ NMR $\left(500 \mathrm{MHz}\right.$, DMSO- $\left.d_{6}\right)$

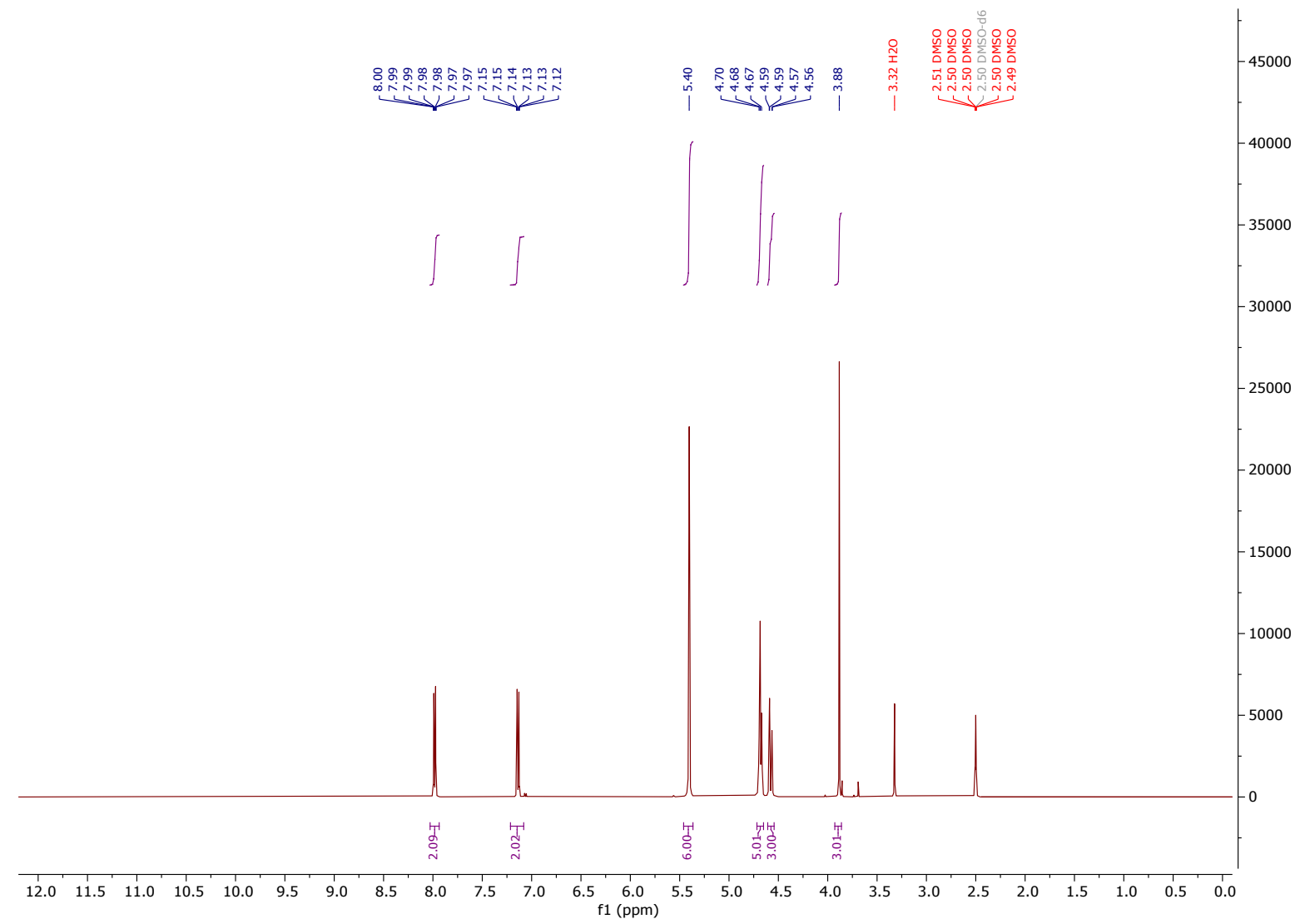

${ }^{13} \mathrm{C}$ NMR (126 MHz, DMSO- $d_{6}$ )

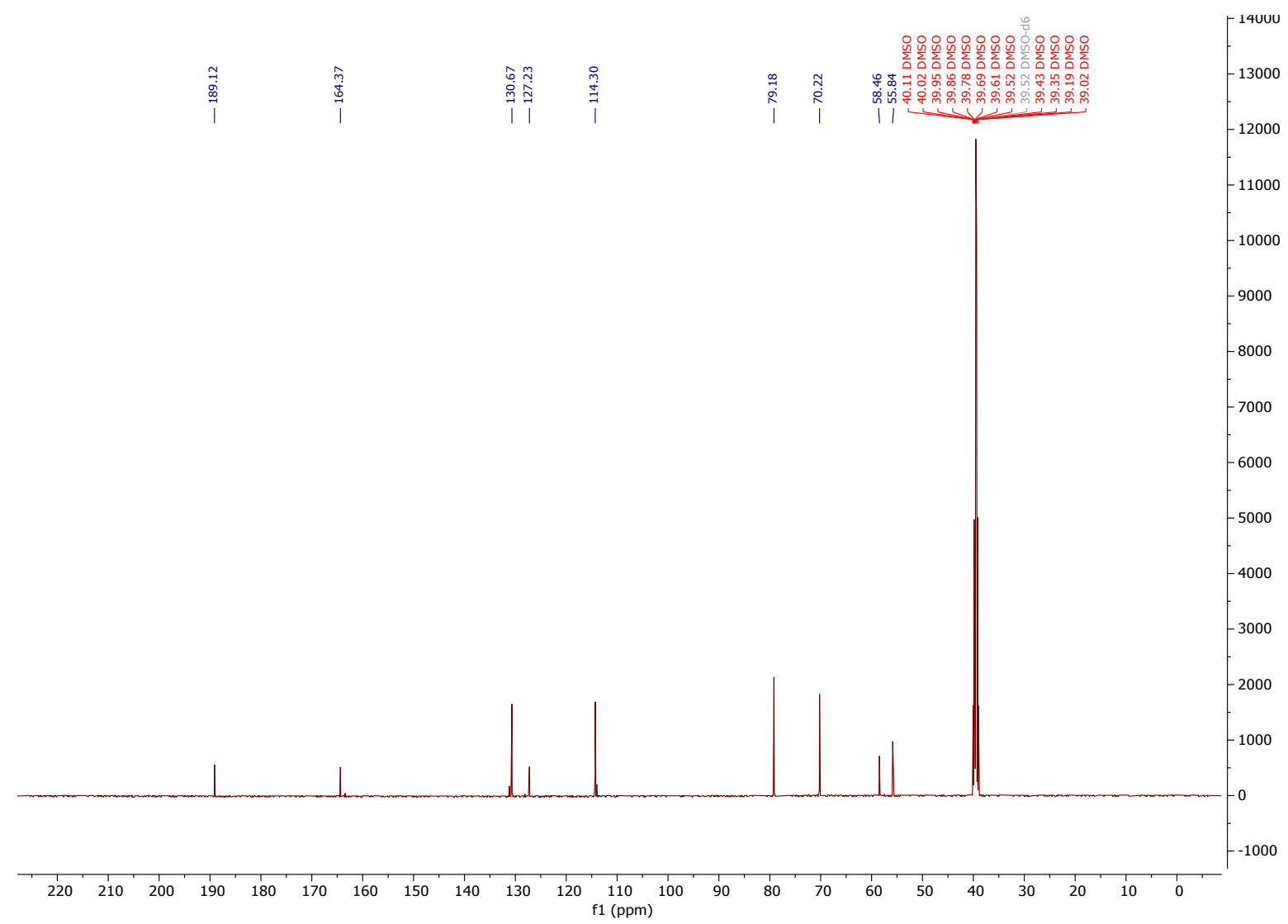


<smiles>O=C(C[N+]12CN3CN(CN(C3)C1)C2)c1ccc(F)cc1</smiles>

1-(2-(4-Fluorophenyl)-2-oxoethyl)-1,3,5,7-tetraazaadamantan-1-ium bromide was synthesised according to General Procedure 2 using hexamethylenetetramine $(701 \mathrm{mg}$, $5.0 \mathrm{mmol}$ ) and 2-bromo-4'fluoroacetophenone $(1.09 \mathrm{~g}, 5.0 \mathrm{mmol})$. Yield: $90 \%(1.61 \mathrm{~g}$, $4.52 \mathrm{mmol})$.

Chemical Formula: $\mathrm{C}_{14} \mathrm{H}_{18} \mathrm{BrFN}_{4} \mathrm{O}$

Molecular Weight: $357.227 \mathrm{gmol}^{-1}$

Appearance: Off-white solid

${ }^{1} \mathrm{H}$ NMR $\left(500 \mathrm{MHz}, \mathrm{DMSO}^{\left.-d_{6}\right)} \delta=8.13-8.04(\mathrm{~m}, 2 \mathrm{H}), 7.52-7.44(\mathrm{~m}, 2 \mathrm{H}), 5.39(\mathrm{~s}, 6 \mathrm{H})\right.$, $4.73(\mathrm{~s}, 2 \mathrm{H}), 4.68(\mathrm{~d}, J=12.8 \mathrm{~Hz}, 3 \mathrm{H}), 4.59(\mathrm{~d}, J=12.5 \mathrm{~Hz}, 3 \mathrm{H})$.

${ }^{13} \mathrm{C}$ NMR $\left(126 \mathrm{MHz}, \mathrm{DMSO}-d_{6}\right) \delta=189.5,165.8(\mathrm{~d}, J=254.0 \mathrm{HZ}), 131.3(\mathrm{~d}, J=10.0 \mathrm{~Hz})$, $131.2(\mathrm{~d}, J=2.7 \mathrm{~Hz}), 116.2(\mathrm{~d}, J=22.2 \mathrm{~Hz}), 79.3,70.2,58.9$.

FT-ATR $v_{\max }: 2896,1692,1596,1469,1223,1146,1054,1007,938,828,791,651,586$, 497.

HRMS $m / z(E S I)+$ calcd. for $\mathrm{C}_{14} \mathrm{H}_{18} \mathrm{FN}_{4} \mathrm{O}[\mathrm{M}]+$ required 277.1459, found 277.1456. 
${ }^{1} \mathrm{H}$ NMR $\left(500 \mathrm{MHz}\right.$, DMSO- $\left.d_{6}\right)$

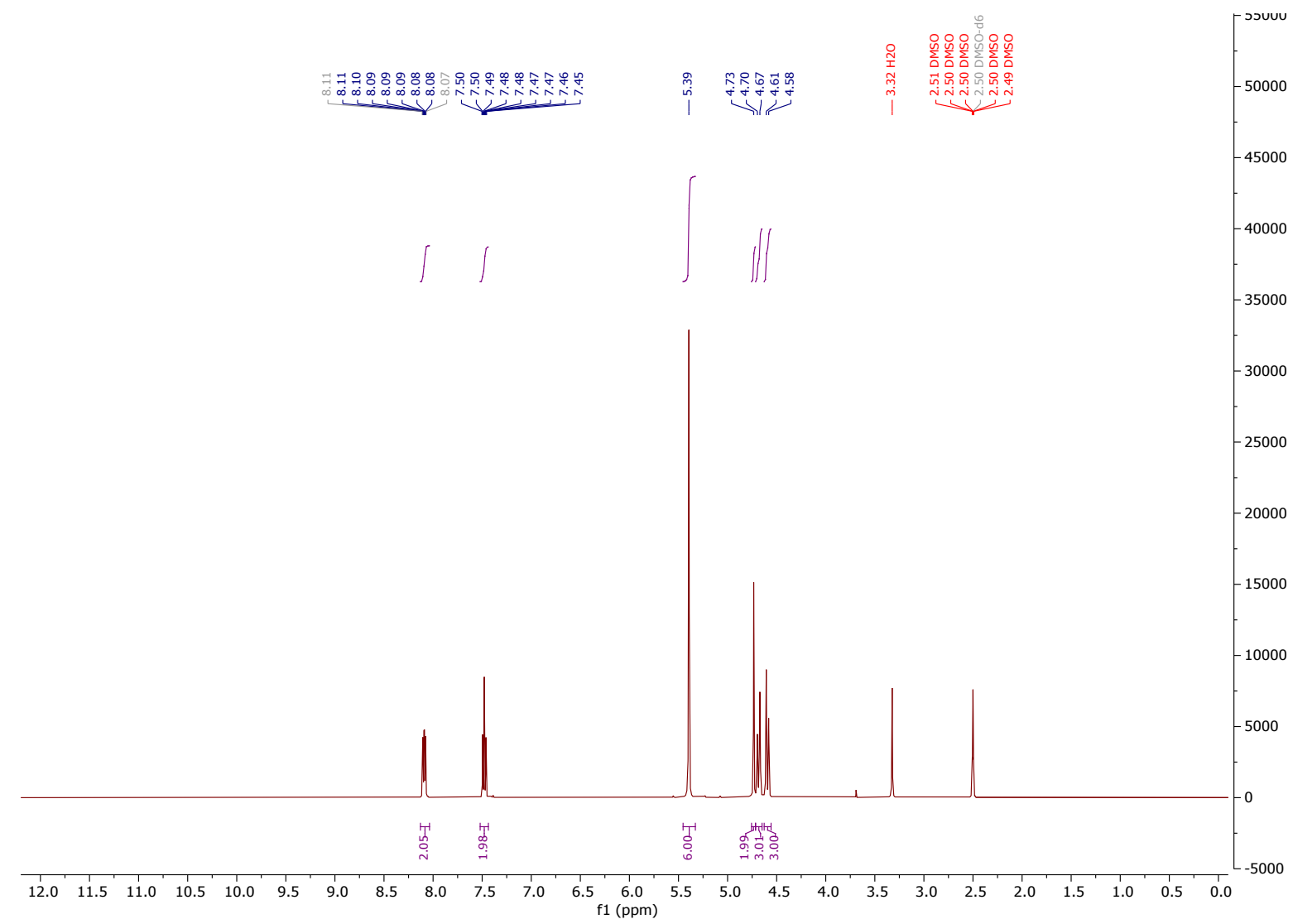

${ }^{13} \mathrm{C}$ NMR (126 MHz, DMSO- $d_{6}$ )

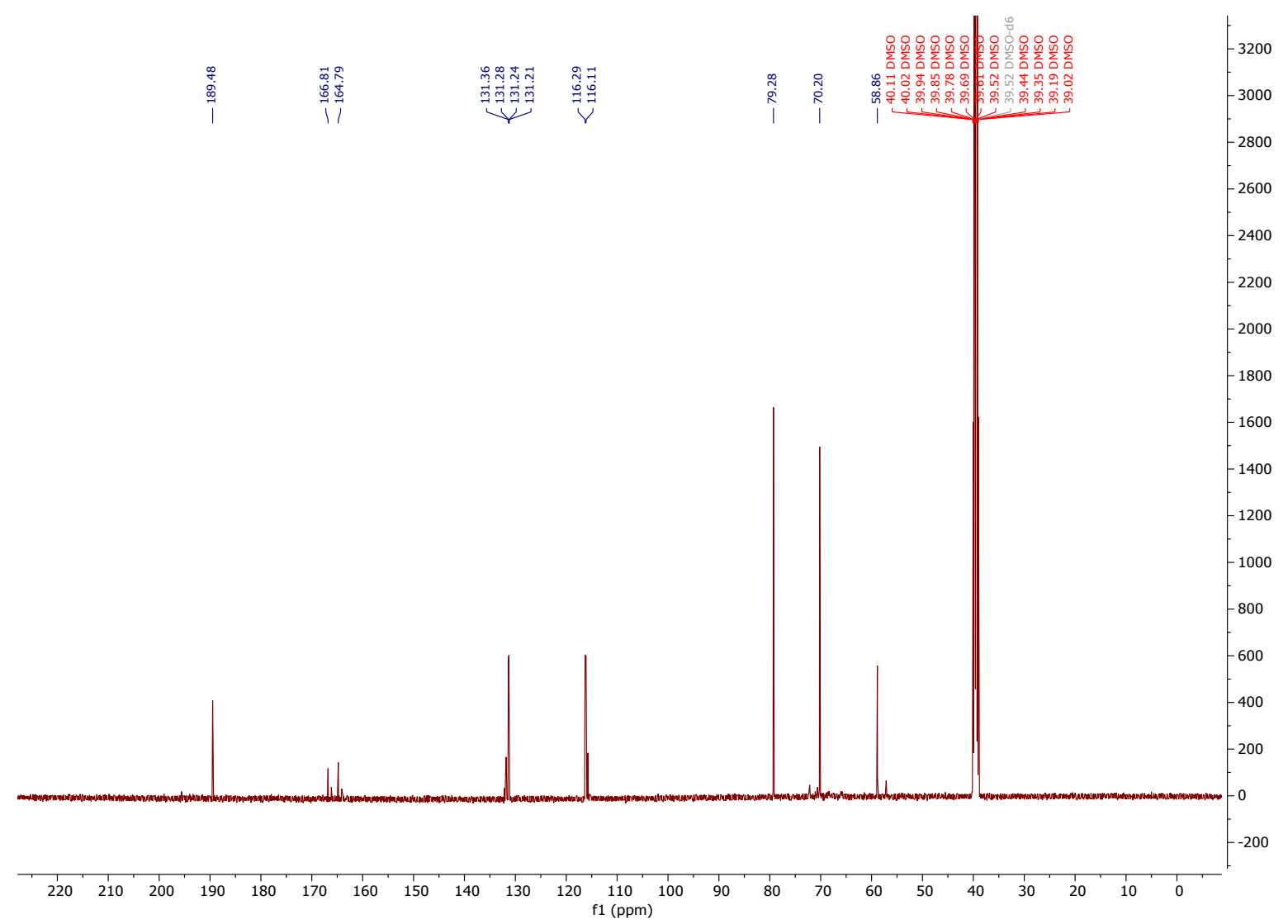


<smiles>O=C(C[N+]12CN3CN(CN(C3)C1)C2)c1cc2ccccc2o1</smiles>

1-(2-(Benzofuran-2-yl)-2-oxoethyl)-1,3,5,7-tetraazaadamantan-1-ium bromide was synthesised according to General Procedure 2 using hexamethylenetetramine $(612 \mathrm{mg}$, $4.37 \mathrm{mmol}$ ) and 2-(bromoacetyl)benzofuran $(1.05 \mathrm{~g}, 4.37 \mathrm{mmol})$. Yield: $92 \%(1.52 \mathrm{~g}$, $4.12 \mathrm{mmol})$.

Chemical Formula: $\mathrm{C}_{16} \mathrm{H}_{19} \mathrm{BrN}_{4} \mathrm{O}_{2}$

Molecular Weight: $379.26 \mathrm{gmol}^{-1}$

Appearance: Yellow Solid

${ }^{1} \mathrm{H}$ NMR $\left(500 \mathrm{MHz}\right.$, DMSO- $\left.d_{6}\right) \delta=8.15(\mathrm{~s}, 1 \mathrm{H}), 7.94(\mathrm{~d}, J=7.9 \mathrm{~Hz}, 1 \mathrm{H}), 7.80(\mathrm{~d}, J=8.4$ $\mathrm{Hz}, 1 \mathrm{H}), 7.65(\mathrm{t}, J=7.8 \mathrm{~Hz}, 1 \mathrm{H}), 7.45(\mathrm{t}, J=7.5 \mathrm{~Hz}, 1 \mathrm{H}), 5.41(\mathrm{~s}, 6 \mathrm{H}), 4.69(\mathrm{~d}, J=12.8$ $\mathrm{Hz}, 3 \mathrm{H}), 4.64-4.52(\mathrm{~m}, 5 \mathrm{H})$.

${ }^{13} \mathrm{C}$ NMR $\left(126 \mathrm{MHz}\right.$, DMSO- $\left.d_{6}\right) \delta=187.1,155.0,150.8,128.9,126.6,124.3,123.9,115.8$, $112.3,72.4,70.7,57.3$.

FT-ATR $v_{\max } 2958,1695,1561,1465,1398,1352,1275,1226,1138,1059,1002,871$, $827,777,645,497$.

HRMS: $m / z$ (ESI) ${ }^{+}$calcd. for $\mathrm{C}_{16} \mathrm{H}_{19} \mathrm{~N}_{4} \mathrm{O}_{2}[\mathrm{M}]^{+}$required 299.1503, found 299.1499. 
${ }^{1} \mathrm{H}$ NMR $\left(500 \mathrm{MHz}, \mathrm{DMSO}-d_{6}\right)$

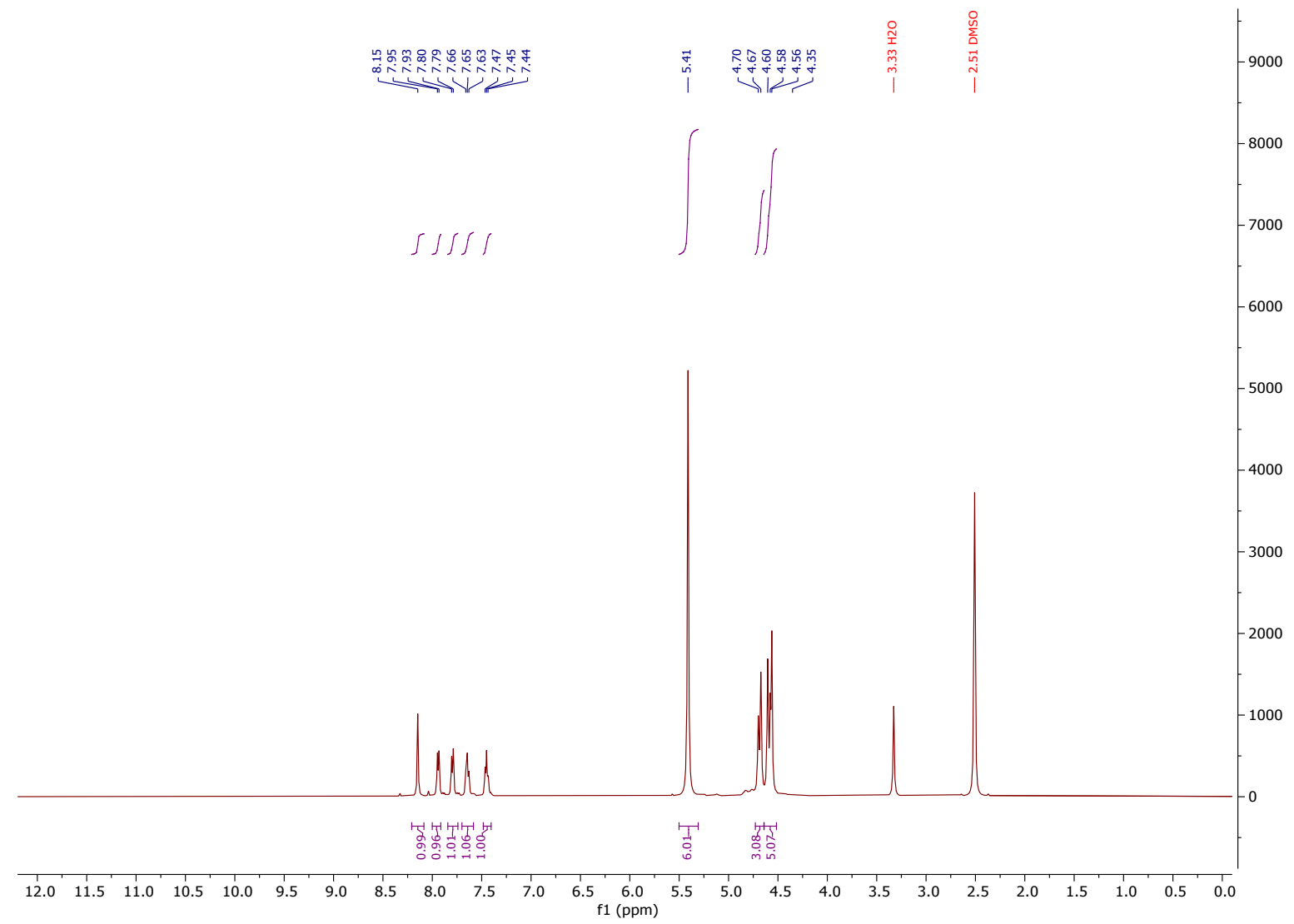

${ }^{13} \mathrm{C}$ NMR (126 MHz, DMSO- $\left.d_{6}\right)$

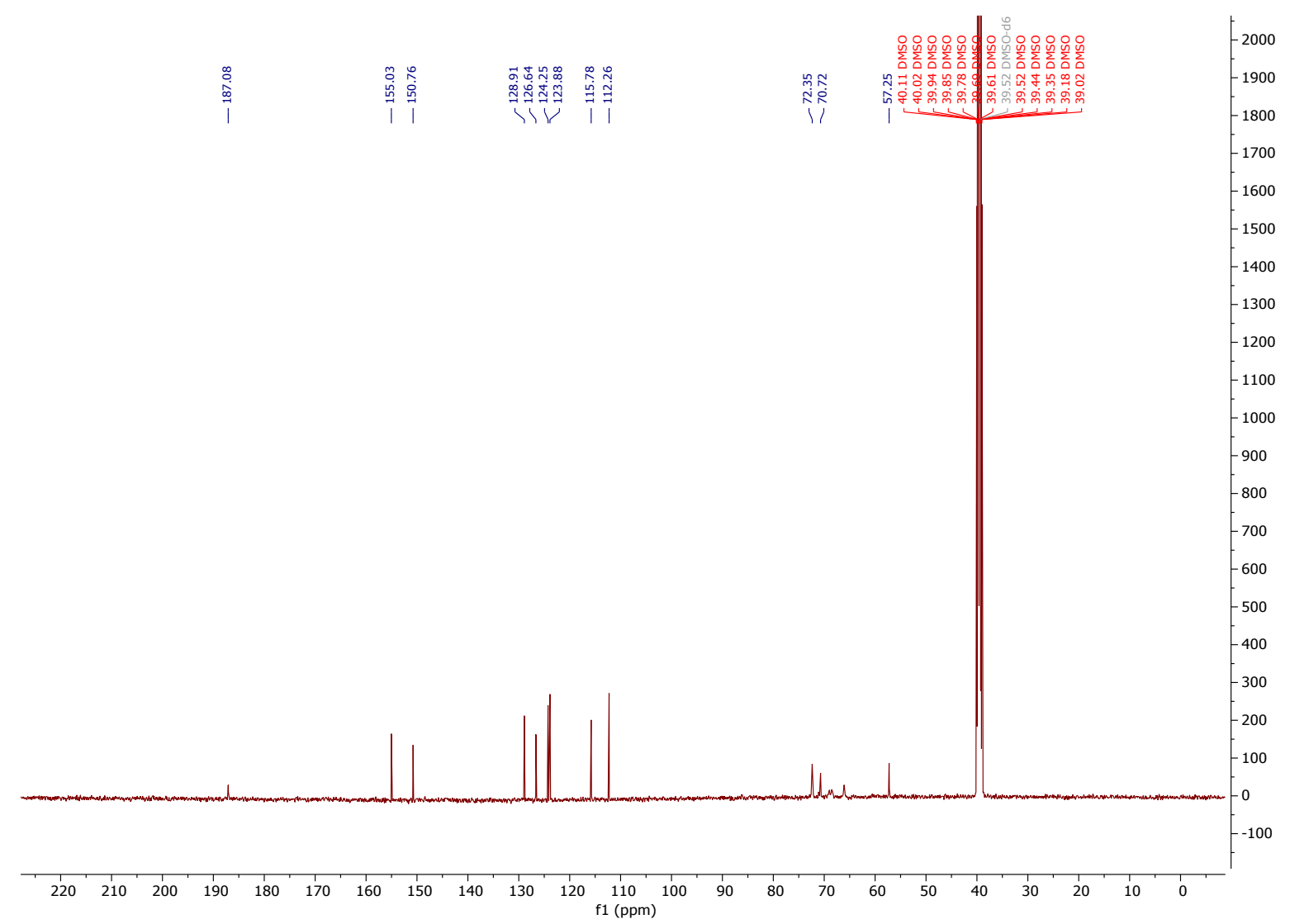


<smiles>O=C(C[N+]12CN3CN(CN(C3)C1)C2)C12CC3CC(CC(C3)C1)C2</smiles>

1-(2-(Adamantan-1-yl)-2-oxoethyl)-1,3,5,7-tetraazaadamantan-1-ium bromide was synthesised according to General Procedure 2 using hexamethylenetetramine $(0.701 \mathrm{~g}$, $5.0 \mathrm{mmol})$ and 1-adamantyl bromoethyl ketone $(1.286 \mathrm{~g}, 5.0 \mathrm{mmol})$. Yield: $72 \%(1.422$ $\mathrm{g}, 3.58 \mathrm{mmol})$.

Chemical Formula: $\mathrm{C}_{18} \mathrm{H}_{29} \mathrm{BrN}_{4} \mathrm{O}$

Molecular Weight: $397.361 \mathrm{gmol}^{-1}$

Appearance: White solid

${ }^{1} \mathrm{H}$ NMR $\left(500 \mathrm{MHz}, \mathrm{DMSO}-d_{6}\right) \delta=5.29(\mathrm{~s}, 6 \mathrm{H}), 4.65(\mathrm{~d}, J=12.7 \mathrm{~Hz}, 3 \mathrm{H}), 4.51(\mathrm{~d}, J=$ $12.5 \mathrm{~Hz}, 3 \mathrm{H}), 4.27(\mathrm{~s}, 2 \mathrm{H}), 2.02(\mathrm{p}, J=3.1 \mathrm{~Hz}, 3 \mathrm{H}), 1.79(\mathrm{~d}, J=3.0 \mathrm{~Hz}, 6 \mathrm{H}), 1.71(\mathrm{dt}, J$ $=12.5,3.0 \mathrm{~Hz}, 3 \mathrm{H}), 1.64(\mathrm{dq}, J=12.5,2.0 \mathrm{~Hz}, 3 \mathrm{H})$.

${ }^{13} \mathrm{C}$ NMR $\left(126 \mathrm{MHz}, \mathrm{DMSO}-d_{6}\right) \delta=207.4,79.2$, 70.7, 57.6, 46.6, 37.1, 36.2, 27.5.

FT-ATR $v_{\max } 2892,2844,1697,1460,1268,1231,1138,1047,1003,819,781,645$, 494.

HRMS $m / z(E S I)^{+}$calcd. for $\mathrm{C}_{18} \mathrm{H}_{29} \mathrm{~N}_{4} \mathrm{O}[\mathrm{M}]^{+}$required 317.2336, found 317.2331. 
${ }^{1} \mathrm{H}$ NMR $\left(500 \mathrm{MHz}\right.$, DMSO- $\left.d_{6}\right)$

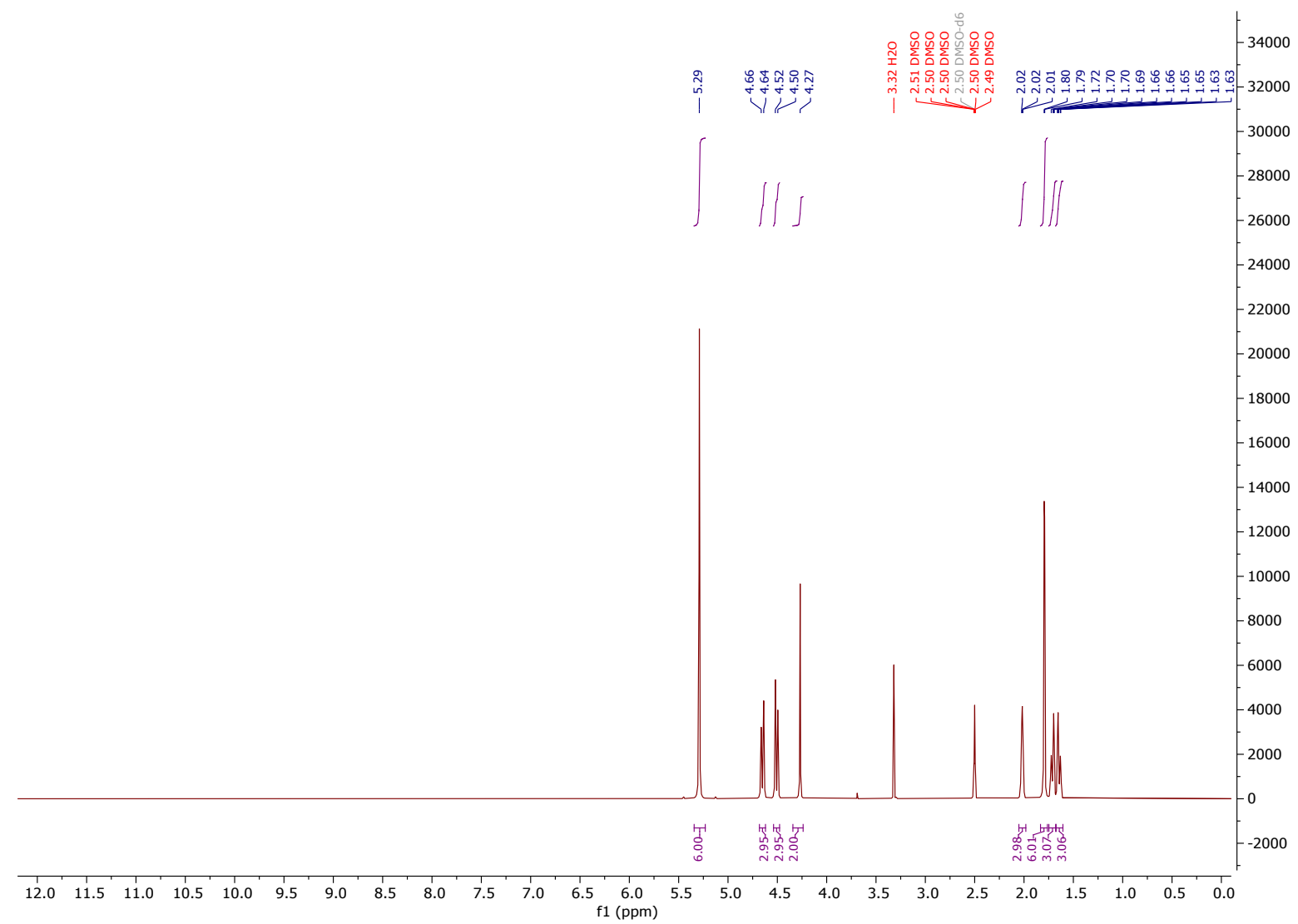

${ }^{13} \mathrm{C}$ NMR (126 MHz, DMSO- $d_{6}$ )

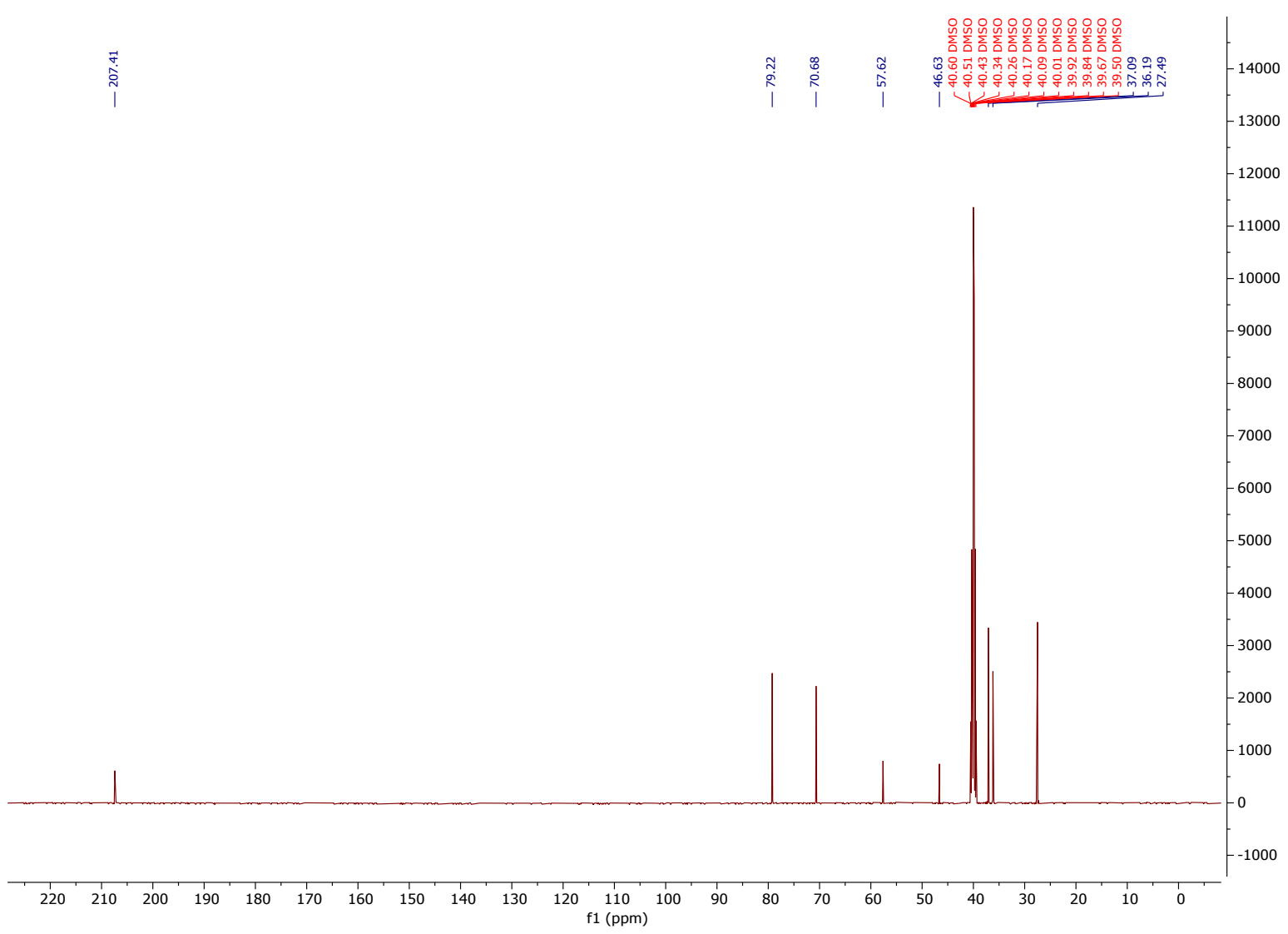


1-((5-(Methoxycarbonyl)pyridin-2-yl)methyl)-1,3,5,7-tetraazaadamantan-1-ium bromide 21<smiles></smiles>

1-((5-(Methoxycarbonyl)pyridin-2-yl)methyl)-1,3,5,7-tetraazaadamantan-1-ium bromide was synthesised according to General Procedure 2 using hexamethylenetetramine (306 $\mathrm{mg}, 2.2 \mathrm{mmol}$ ) and methyl 6-(bromomethyl)nicotinate (506 mg, $2.2 \mathrm{mmol}$ ). Yield: $98 \%$ (803 $\mathrm{mg}, 2.16 \mathrm{mmol}$ ).

Chemical Formula: $\mathrm{C}_{14} \mathrm{H}_{20} \mathrm{BrN}_{5} \mathrm{O}_{2}$

Molecular Weight: $370.251 \mathrm{gmol}^{-1}$

Appearance: Red solid

${ }^{1} \mathrm{H}$ NMR $\left(500 \mathrm{MHz}\right.$, DMSO- $\left.d_{6}\right) \delta=9.18(\mathrm{~d}, J=2.2 \mathrm{~Hz}, 1 \mathrm{H}), 8.45(\mathrm{dd}, J=8.0,2.3 \mathrm{~Hz}, 1 \mathrm{H})$, $7.77(\mathrm{~d}, J=8.0 \mathrm{~Hz}, 1 \mathrm{H}), 5.20(\mathrm{~s}, 6 \mathrm{H}), 4.59(\mathrm{~d}, J=12.6 \mathrm{~Hz}, 3 \mathrm{H}), 4.45(\mathrm{~d}, J=12.3 \mathrm{~Hz}$, $3 \mathrm{H}), 4.24(\mathrm{~s}, 2 \mathrm{H}), 3.93(\mathrm{~s}, 3 \mathrm{H})$.

${ }^{13} \mathrm{C}$ NMR $\left(126 \mathrm{MHz}\right.$, DMSO- $\left.d_{6}\right) \delta=164.9,151.7,150.3,138.4,127.3,126.1,78.4,69.7$, $59.7,52.7$.

FT-ATR $v_{\max } 2984,2942,1752,1598,1447,1275,1117,1034,1002,941,824,777$, $651,501$.

HRMS $m / z$ (ESI) ${ }^{+}$calcd. for $\mathrm{C}_{14} \mathrm{H}_{20} \mathrm{~N}_{5} \mathrm{O}_{2}[\mathrm{M}]^{+}$required 290.1611 , found 290.1612 
${ }^{1} \mathrm{H}$ NMR $\left(500 \mathrm{MHz}\right.$, DMSO- $\left.d_{6}\right)$

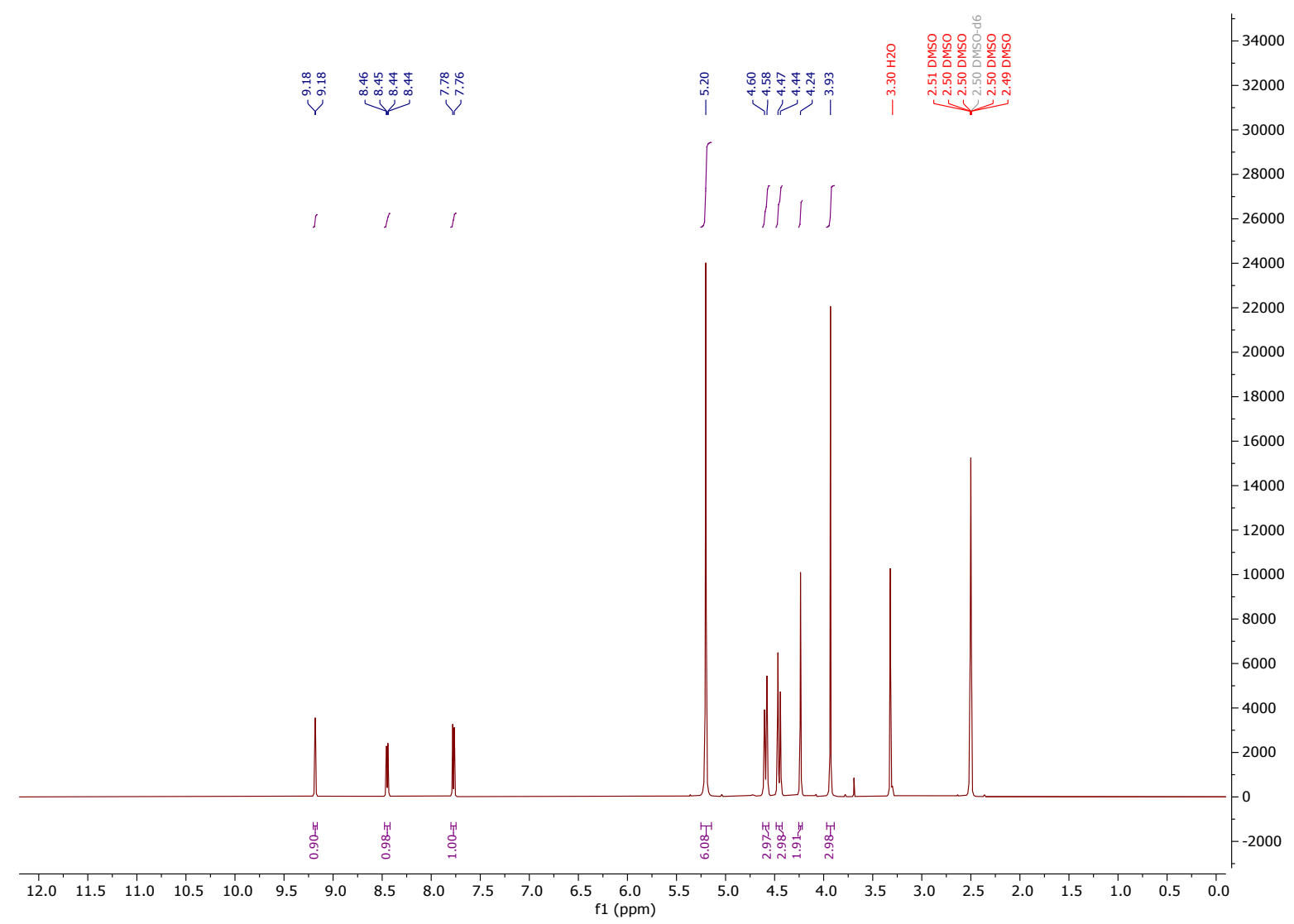

${ }^{13} \mathrm{C}$ NMR (126 MHz, DMSO- $d_{6}$ )

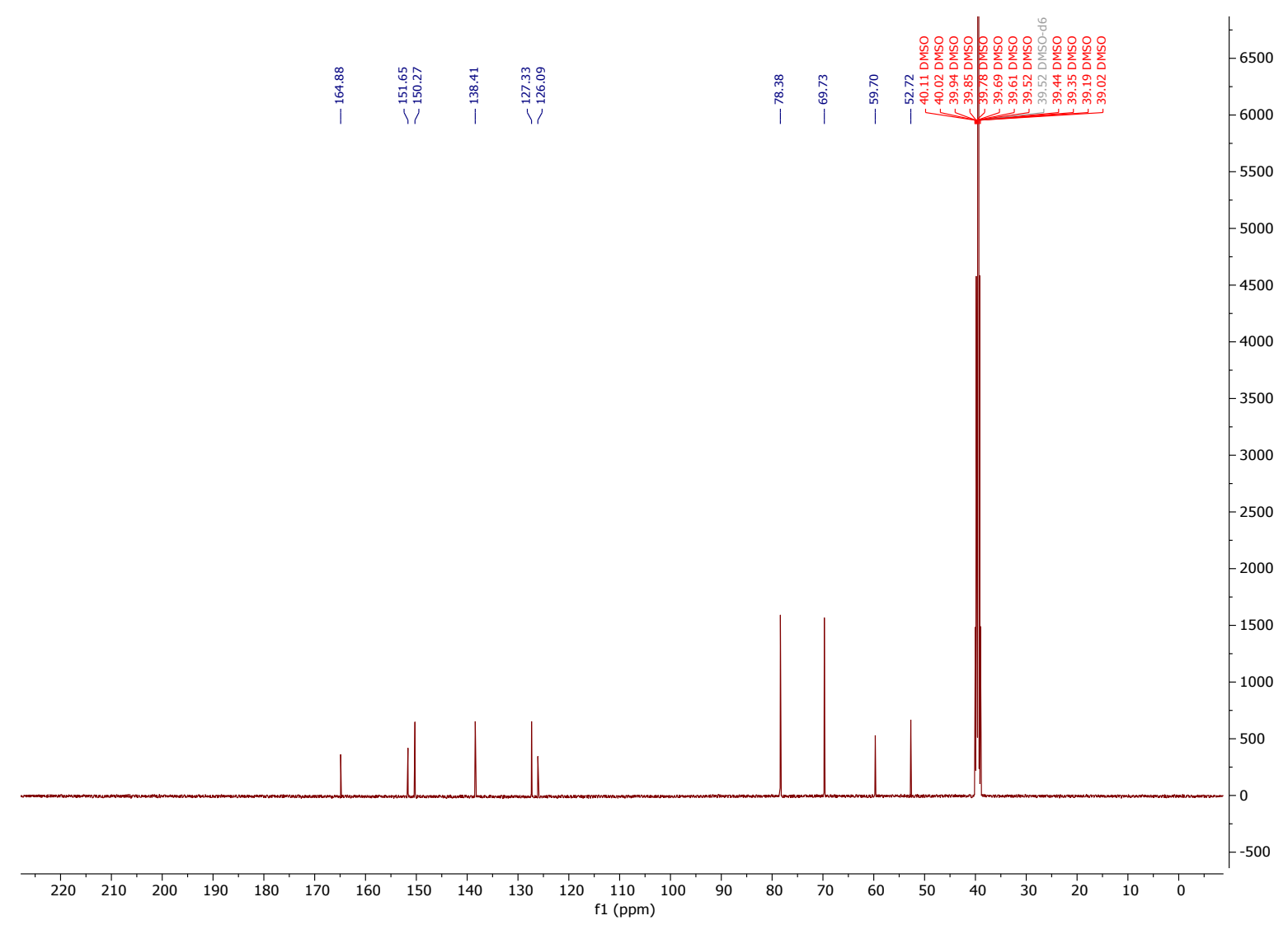


<smiles>O=C(C[N+]12CN3CN(CN(C3)C1)C2)c1ccccn1</smiles>

1-(2-Oxo-2-(pyridin-1-ium-2-yl)ethyl)-1,3,5,7-tetraazaadamantan-1-ium hydrobromide was synthesised according to General Procedure 2 using hexamethylenetetramine $(505 \mathrm{mg}, 3.6 \mathrm{mmol}$ ) and 2-(bromoacetyl)pyridine hydrobromide $(1.01 \mathrm{~g}, 3.6 \mathrm{mmol})$. Yield: $53 \%$ (798 $\mathrm{mg}, 1.89 \mathrm{mmol})$.

Chemical Formula: $\mathrm{C}_{13} \mathrm{H}_{19} \mathrm{Br}_{2} \mathrm{~N}_{5} \mathrm{O}_{2}$

Molecular Weight: $421.137 \mathrm{gmol}^{-1}$

Appearance: Yellow solid

${ }^{1} \mathrm{H}$ NMR $\left(500 \mathrm{MHz}\right.$, DMSO- $\left.d_{6}\right) \delta=8.77(\mathrm{~d}, J=4.7 \mathrm{~Hz}, 1 \mathrm{H}), 8.15-8.09(\mathrm{~m}, 1 \mathrm{H}), 8.06(\mathrm{~d}$, $J=7.8 \mathrm{~Hz}, 1 \mathrm{H}), 7.79(\mathrm{dd}, J=7.5,4.7 \mathrm{~Hz}, 1 \mathrm{H}), 5.41(\mathrm{~s}, 6 \mathrm{H}), 4.80(\mathrm{~s}, 2 \mathrm{H}), 4.68(\mathrm{~d}, J=$ $12.7 \mathrm{~Hz}, 3 \mathrm{H}), 4.58(\mathrm{~d}, J=12.7 \mathrm{~Hz}, 3 \mathrm{H})$.

${ }^{13} \mathrm{C}$ NMR $\left(126 \mathrm{MHz}, \mathrm{DMSO}-d_{6}\right) \delta=191.3,151.1,149.2,138.1,129.1,121.9,79.2,71.1$, $70.2,58.8$.

FT-ATR $v_{\max }: 2535,1709,1458,1390,1278,1210,1056,1005,936,867,779,652,496$.

HRMS $m / z$ (ESI) ${ }^{+}$calcd. for $\mathrm{C}_{13} \mathrm{H}_{18} \mathrm{~N}_{5} \mathrm{O}[\mathrm{M}]^{+}$required 260.1506, found 260.1504. 
${ }^{1} \mathrm{H}$ NMR $\left(500 \mathrm{MHz}, \mathrm{DMSO}-d_{6}\right)$

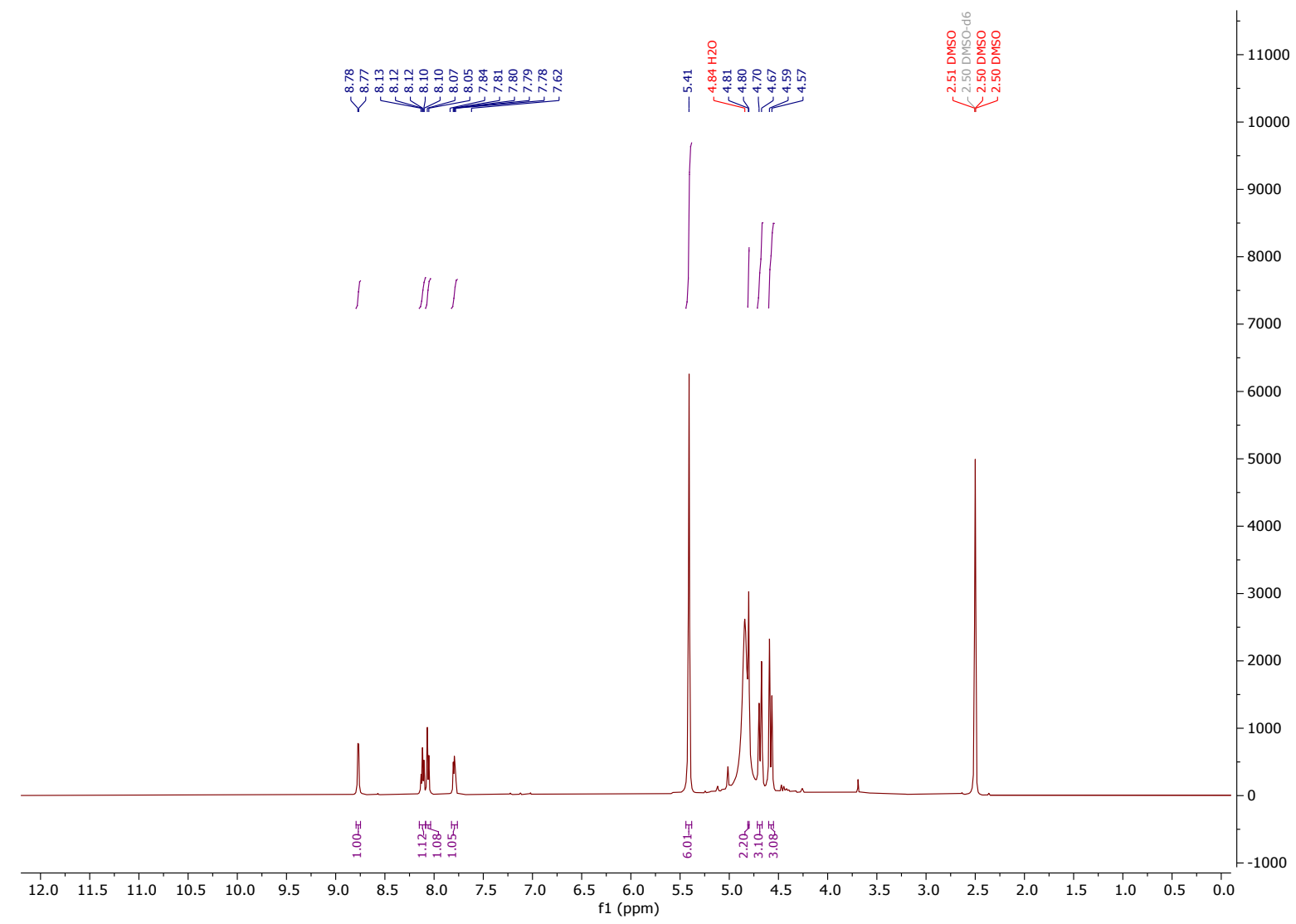

${ }^{13} \mathrm{C}$ NMR (126 MHz, DMSO- $\left.d_{6}\right)$

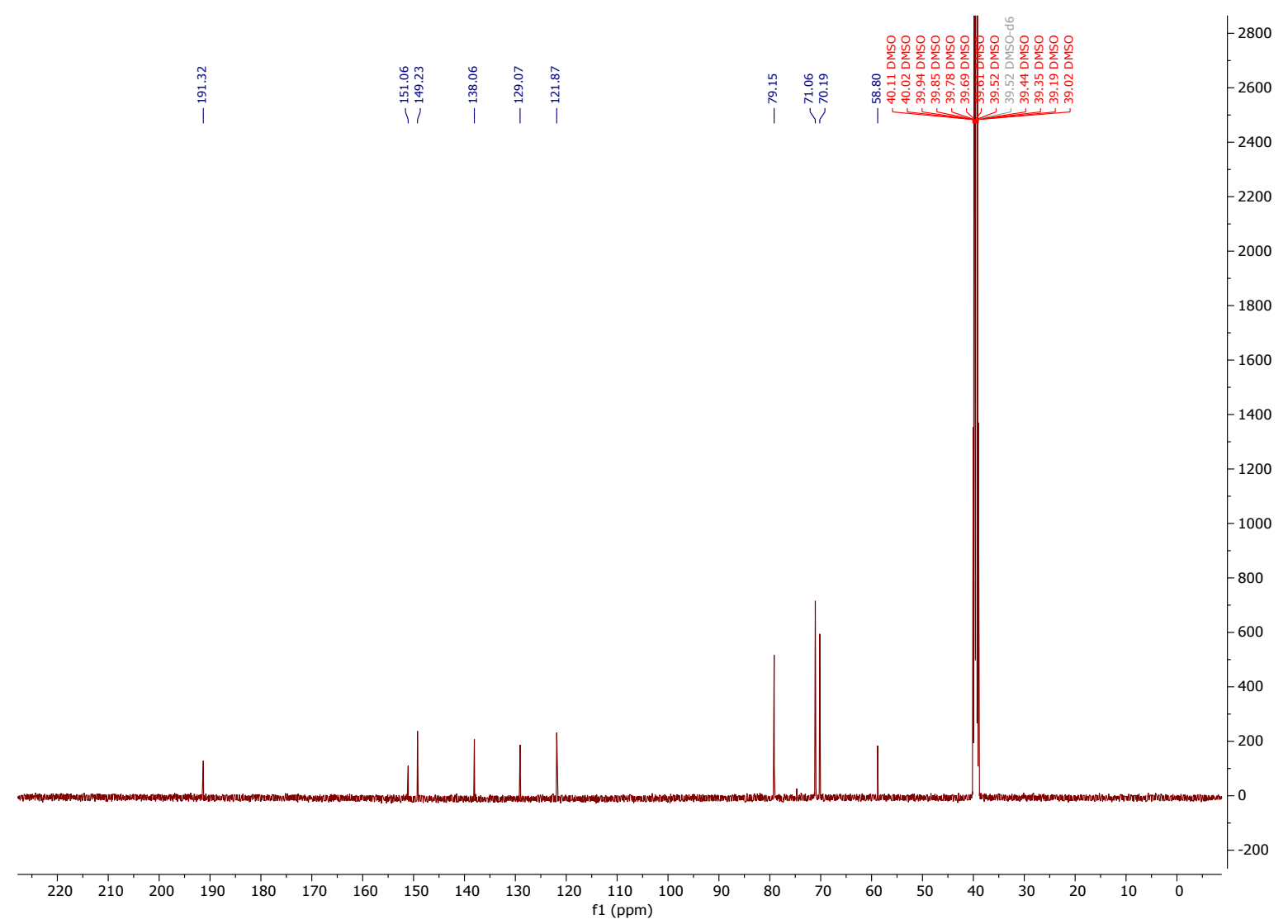


1-(2-(1-(tert-Butoxycarbonyl)piperidin-4-yl)-2-oxoethyl)-1,3,5,7-tetraazaadamantan-1ium bromide 23<smiles>CC(C)(C)OC(=O)N1CCC(C(=O)C[N+]23CC4CN(CN(C4)C2)C3)CC1</smiles>

1-(2-(1-(tert-butoxycarbonyl)piperidin-4-yl)-2-oxoethyl)-1,3,5,7-tetraazaadamantan-1ium bromide was synthesised according to General Procedure 2 using hexamethylenetetramine (701 $\mathrm{mg}, 5.0 \mathrm{mmol})$ and tert-butyl 4-(2bromoacetyl)piperidine-1-carboxylate $(1.53 \mathrm{~g}, 5.0 \mathrm{mmol})$. Yield: $67 \%(1.49 \mathrm{~g}, 3.34$ $\mathrm{mmol}$ ).

Chemical Formula: $\mathrm{C}_{18} \mathrm{H}_{32} \mathrm{BrN}_{5} \mathrm{O}_{3}$

Molecular Weight: $446.390 \mathrm{gmol}^{-1}$

Appearance: Pale yellow solid

$\mathrm{T}_{\mathrm{m}}{ }^{\circ} \mathrm{C}: 169^{\circ} \mathrm{C}$.

${ }^{1} \mathrm{H}$ NMR $\left(500 \mathrm{MHz}, \mathrm{D}_{2} \mathrm{O}\right) \delta=5.00-4.83(\mathrm{~m}, 7 \mathrm{H}), 4.56(\mathrm{t}, J=8.7 \mathrm{~Hz}, 1 \mathrm{H}), 4.40(\mathrm{~d}, J=$ $13.8 \mathrm{~Hz}, 1 \mathrm{H}), 4.33(\mathrm{~d}, J=13.6 \mathrm{~Hz}, 1 \mathrm{H}), 4.18(\mathrm{~d}, J=13.8 \mathrm{~Hz}, 2 \mathrm{H}), 4.07(\mathrm{~d}, J=13.4 \mathrm{~Hz}$, $2 \mathrm{H}), 3.84(\mathrm{dd}, J=15.3,9.3 \mathrm{~Hz}, 1 \mathrm{H}), 3.63(\mathrm{dd}, J=15.2,8.1 \mathrm{~Hz}, 1 \mathrm{H}), 2.99(\mathrm{tt}, J=11.5$, $3.6 \mathrm{~Hz}, 1 \mathrm{H}), 2.89(\mathrm{t}, J=13.0 \mathrm{~Hz}, 2 \mathrm{H}), 1.90(\mathrm{~d}, J=13.3 \mathrm{~Hz}, 1 \mathrm{H}), 1.84(\mathrm{~d}, J=13.4 \mathrm{~Hz}$, $1 \mathrm{H}), 1.52-1.43(\mathrm{~m}, 11 \mathrm{H})$.

${ }^{13} \mathrm{C}$ NMR $\left(126 \mathrm{MHz}\right.$, DMSO- $d_{6}$, rotamers) $\delta=210.3,154.4,79.7,79.1,72.9,72.7,71.5$, 70.9, 69.1, 66.7, 56.5, 45.1, 28.5, 27.9, 27.6.

FT-ATR $v_{\max } 2862,2522,1689,1451,1304,1169,1100,1025,1003,858,667$.

HRMS $m / z(E S I)+$ calcd. for $\mathrm{C}_{18} \mathrm{H}_{32} \mathrm{~N}_{5} \mathrm{O}_{3}[\mathrm{M}]^{+}$required 366.2492, found 366.2500 
${ }^{1} \mathrm{H}$ NMR $\left(500 \mathrm{MHz}, \mathrm{D}_{2} \mathrm{O}\right)$

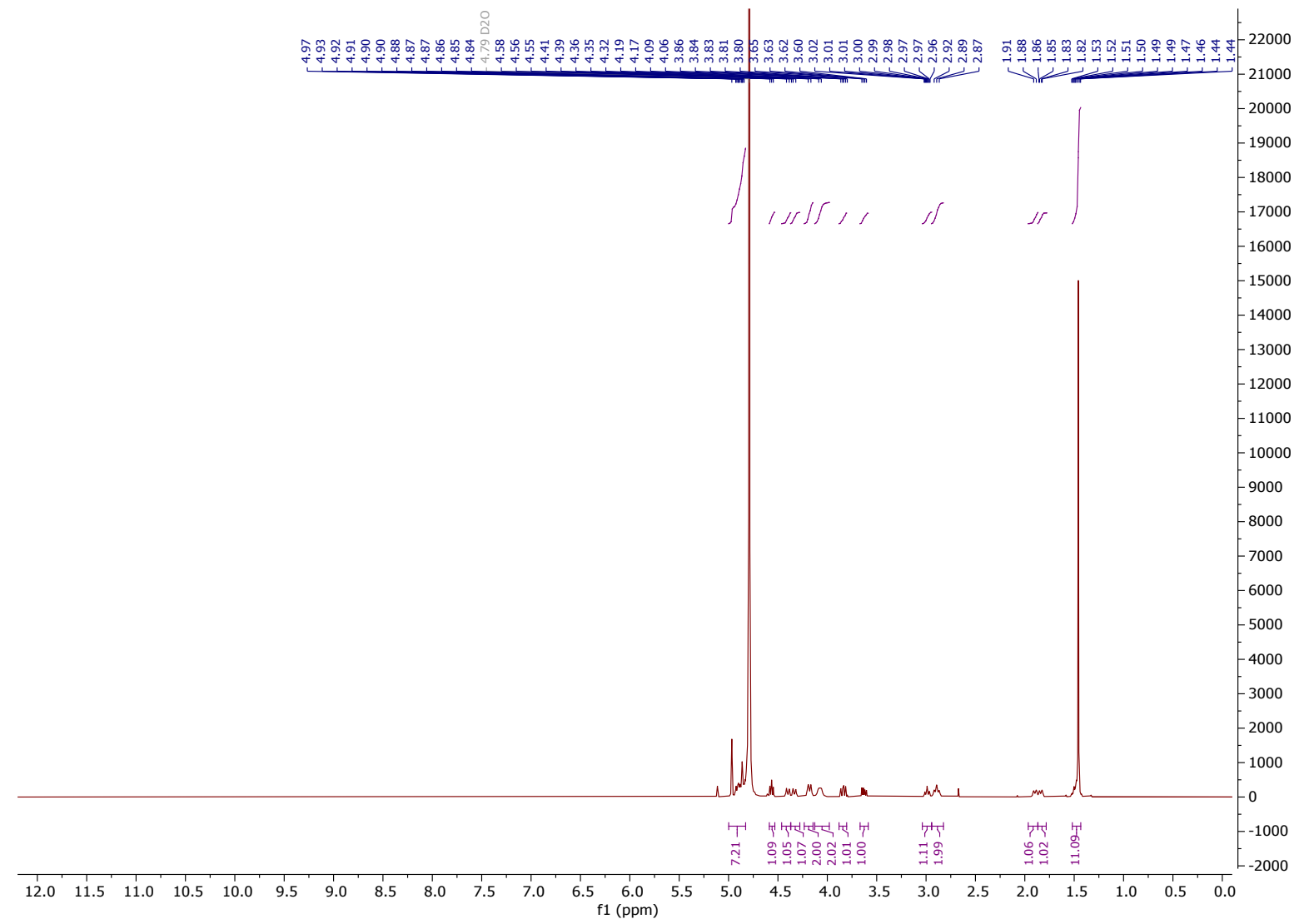

${ }^{13} \mathrm{C}$ NMR (126 MHz, DMSO- $\left.d_{6}\right)$

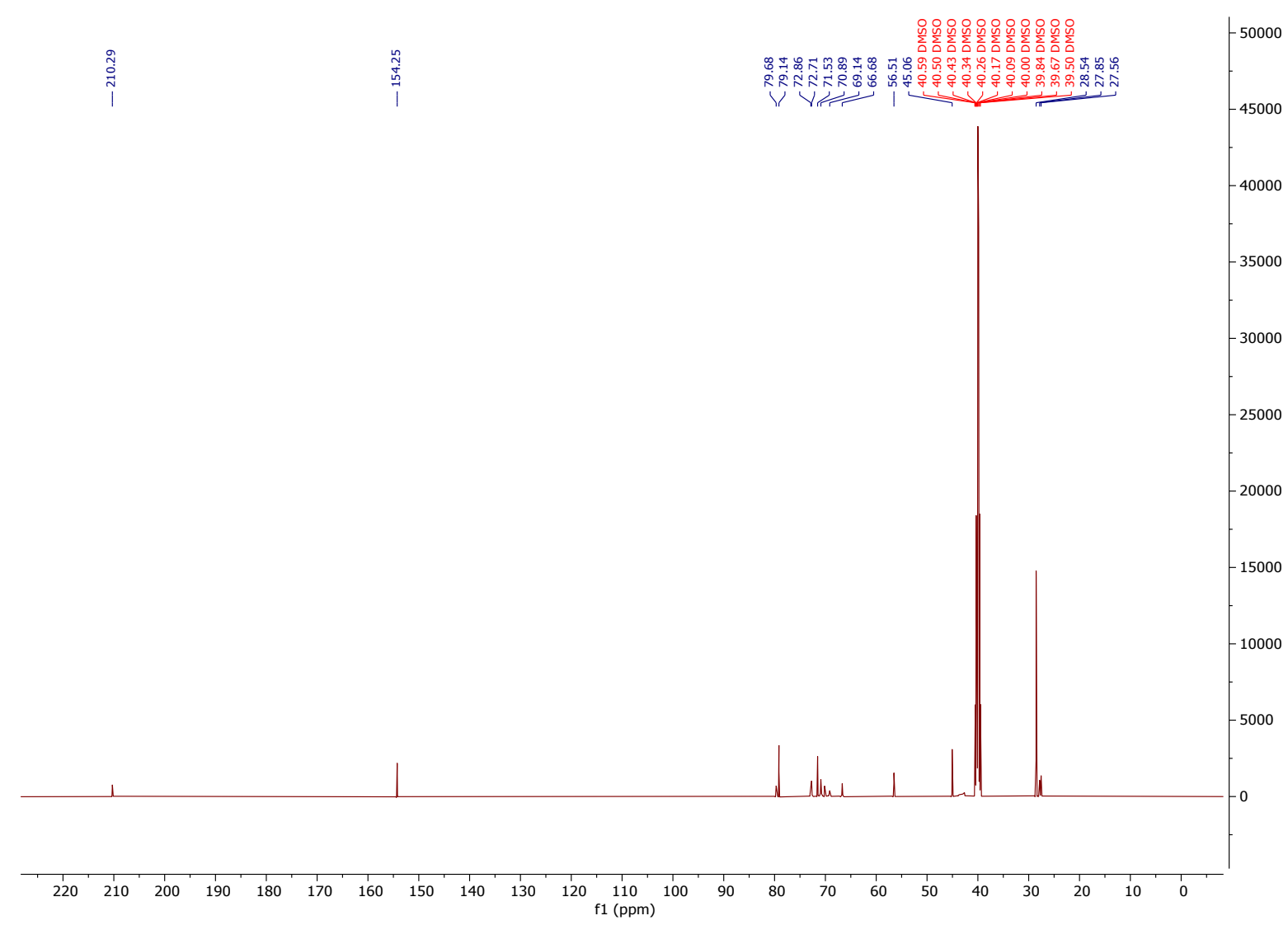




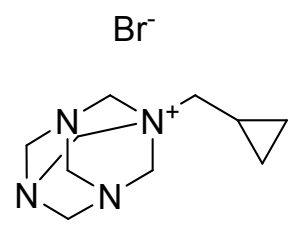

1-(Cyclopropylmethyl)-1,3,5,7-tetraazaadamantan-1-ium bromide was synthesised according to General Procedure 2 using hexamethylenetetramine ( $701 \mathrm{mg}, 5.0 \mathrm{mmol}$ ) and (bromomethyl)cyclopropane $(485 \mu \mathrm{L}, 5.0 \mathrm{mmol})$. Crude mass recovery after filtration: $1.00 \mathrm{~g}$. Purity determined by ${ }^{1} \mathrm{H}-\mathrm{NMR}: 93.0 \mathrm{wt} . \%$.

Yield: $68 \%(0.930 \mathrm{~g}, 3.38 \mathrm{mmol})$.

Chemical Formula: $\mathrm{C}_{10} \mathrm{H}_{19} \mathrm{BrN}_{4}$

Molecular Weight: $275.19 \mathrm{gmol}^{-1}$

Appearance: White solid

${ }^{1} \mathrm{H}$ NMR $\left(400 \mathrm{MHz}, \mathrm{D}_{2} \mathrm{O}\right) \delta=5.12(\mathrm{~s}, 6 \mathrm{H}), 4.66(\mathrm{~s}, 3 \mathrm{H}), 4.56-4.50(\mathrm{~m}, 3 \mathrm{H}), 2.79(\mathrm{~d}, \mathrm{~J}=$ $7.5 \mathrm{~Hz}, 2 \mathrm{H}), 1.14-0.95(\mathrm{~m}, 1 \mathrm{H}), 0.81-0.68(\mathrm{~m}, 2 \mathrm{H}), 0.39-0.25(\mathrm{~m}, 2 \mathrm{H})$.

${ }^{13} \mathrm{C}$ NMR $\left(101 \mathrm{MHz}, \mathrm{D}_{2} \mathrm{O}\right) \delta=78.1,70.1,62.2,4.1,1.6$.

FT-ATR $v_{\max } 2979,2886,2251,1707,1460,1431,1395,1363,1328,1273,1226,1122$, 1072, 1040, 997, 951, 872, 822, 783, 708, 680, 649, 621, 594, 528, 498, 458, 438.

HRMS $m / z$ (ESI) ${ }^{+}$calcd. for $\mathrm{C}_{10} \mathrm{H}_{19} \mathrm{~N}_{4}[\mathrm{M}]^{+}$required 195.1604, found 195.1607. 
${ }^{1} \mathrm{H}$ NMR $\left(400 \mathrm{MHz}, \mathrm{D}_{2} \mathrm{O}\right)$

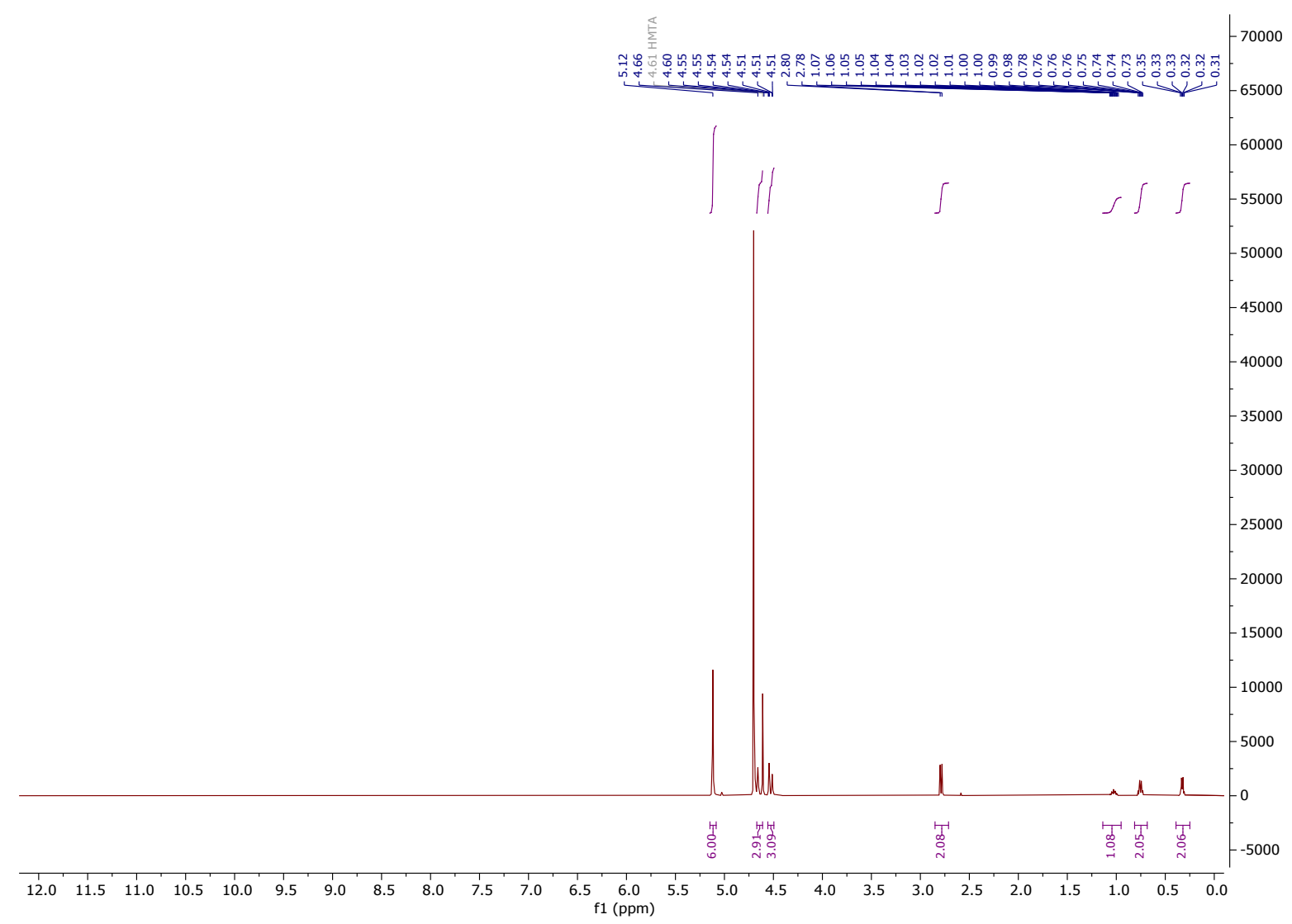

${ }^{13} \mathrm{C}$ NMR $\left(101 \mathrm{MHz}, \mathrm{D}_{2} \mathrm{O}\right)$

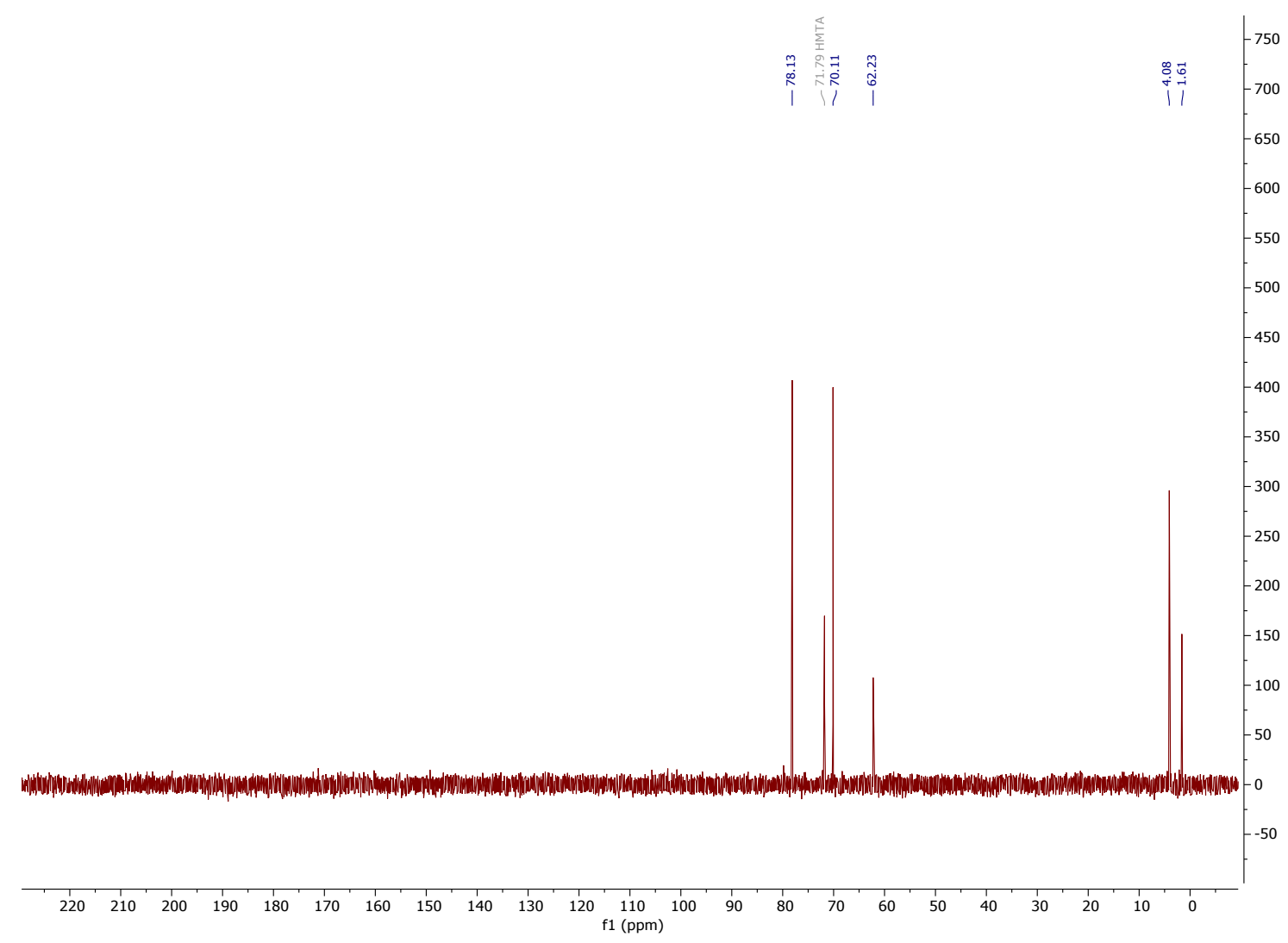


tert-Butyl 2,5-dihydro-1H-pyrrole-1-carboxylate 29<smiles>CC(C)(C)OC(=O)N1CC=CC1</smiles>

tert-Butyl 2,5-dihydro-1H-pyrrole-1-carboxylate was synthesised according to the procedure outlined by Tammana et al. from compound $13^{16}$ and purified by silica gel chromatography using a cyclohexane:EtOAc gradient elution; 0-15\% EtOAc.

Chemical Formula: $\mathrm{C}_{9} \mathrm{H}_{15} \mathrm{NO}_{2}$

Molecular Weight: $169.22 \mathrm{gmol}^{-1}$

Appearance: Pale yellow oil

Yield: $86 \%(5.72 \mathrm{~g}, 33.8 \mathrm{mmol})$

${ }^{1} \mathrm{H}$ NMR $\left(500 \mathrm{MHz}, \mathrm{CDCl}_{3}\right) \delta=5.83-5.67(\mathrm{~m}, 2 \mathrm{H}), 4.15-4.05(\mathrm{~m}, 4 \mathrm{H}), 1.47(\mathrm{~s}, 9 \mathrm{H})$.

${ }^{13} \mathrm{C}$ NMR $\left(126 \mathrm{MHz}, \mathrm{CDCl}_{3}\right) \delta=154.5,126.0,125.9,79.4,53.2,53.0,28.7$.

NMR data was in agreement with the literature. ${ }^{16}$ 
${ }^{1} \mathrm{H}$ NMR $\left(500 \mathrm{MHz}, \mathrm{CDCl}_{3}\right)$

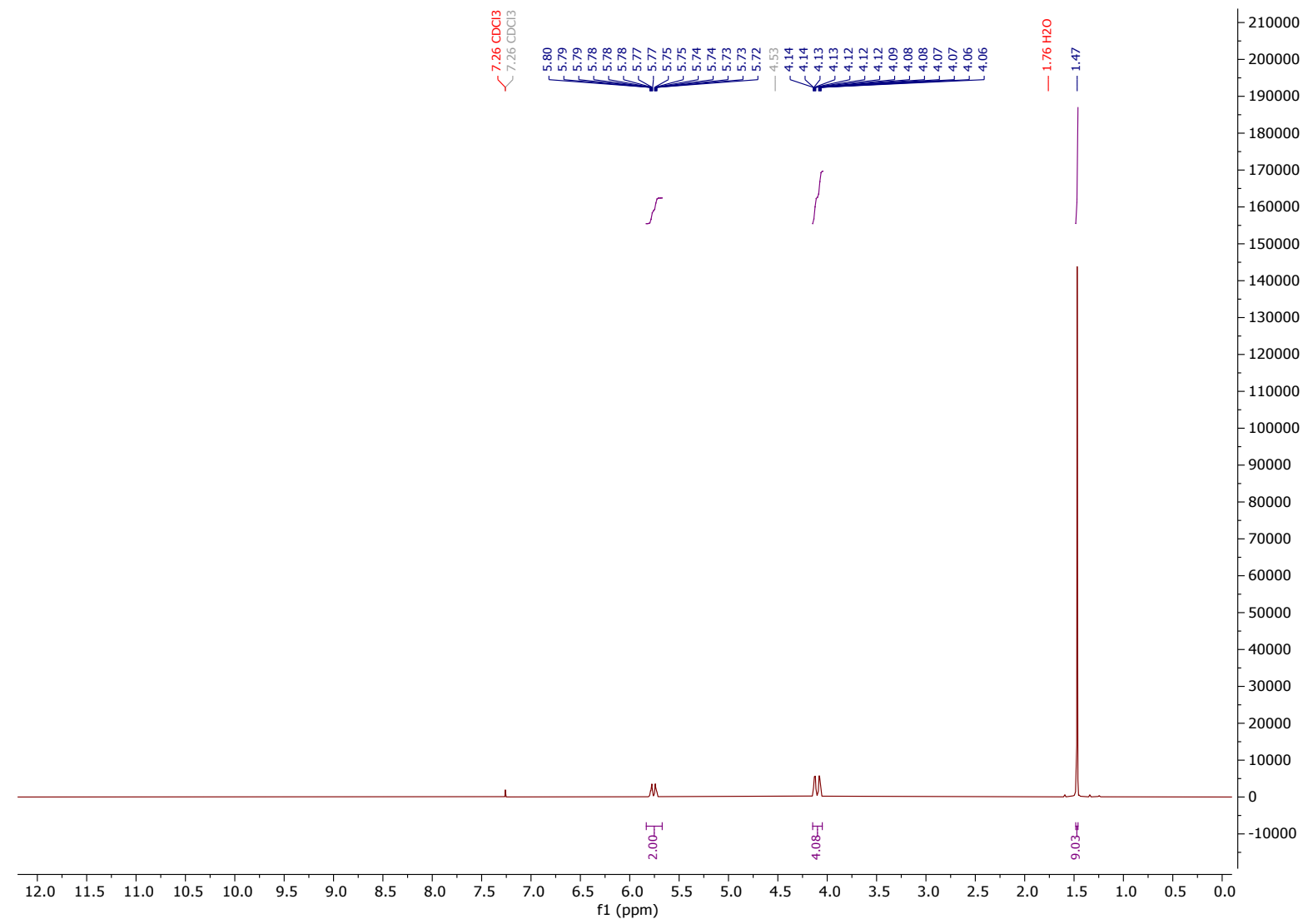

${ }^{13} \mathrm{C}$ NMR $\left(101 \mathrm{MHz}, \mathrm{CDCl}_{3}\right)$

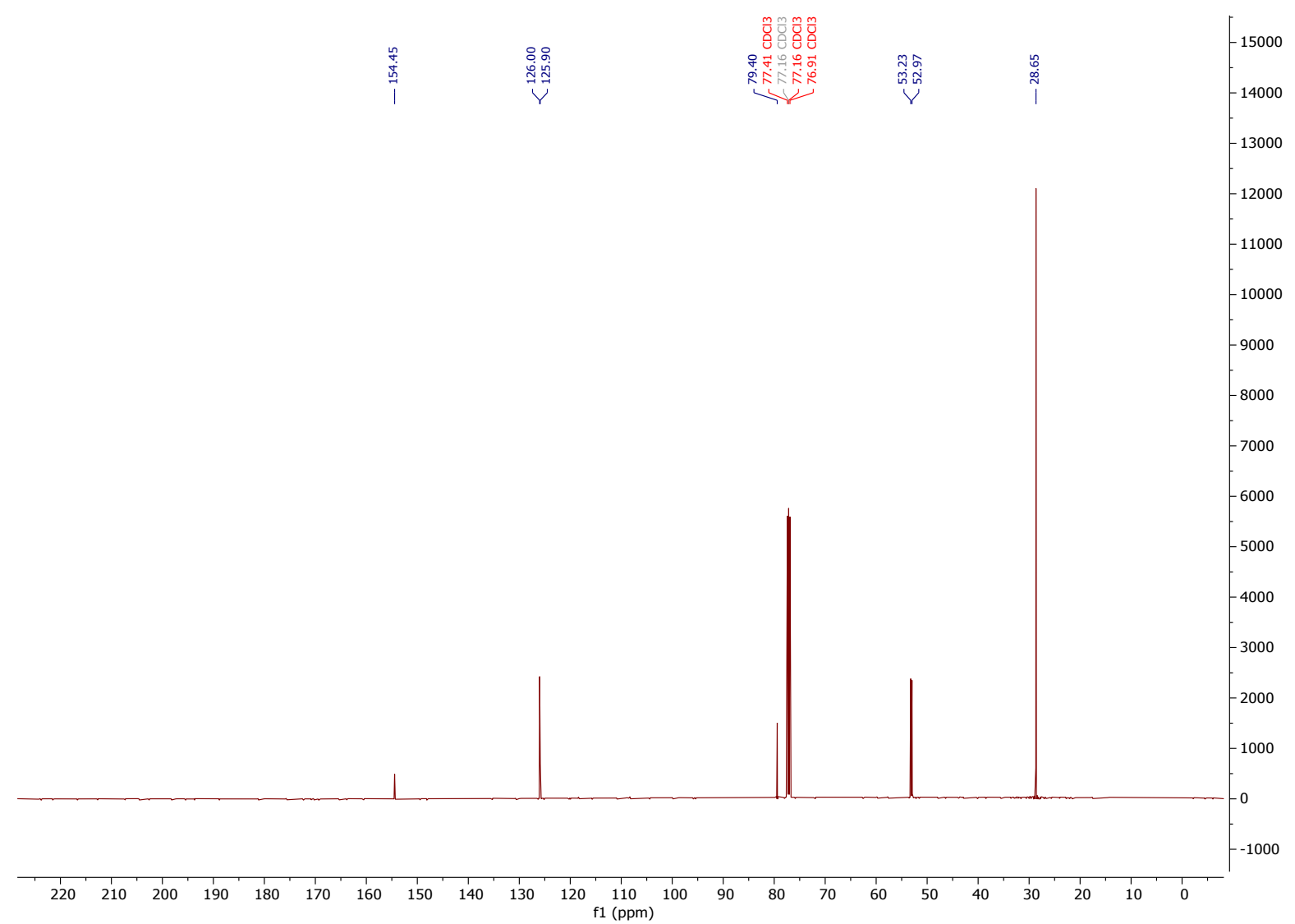


<smiles>NCC(=O)c1ccccc1</smiles>

To a stirred suspension of $\mathbf{1 5}$ in ethanol ( $3 \mathrm{~mL}$ ) was added hydrochlorid acid (concentrated, $300 \mu \mathrm{L}$ ). The suspension was heated to $70^{\circ} \mathrm{C}$ and upon heating clarified to a clear yellow solution. After $1.5 \mathrm{~h}$, the solution was cooled to room temperature and the solvent was removed in vacuo to give the title product as a pale yellow solid mixed with $\mathrm{NH}_{4} \mathrm{Cl}$. ${ }^{1} \mathrm{H}-$ NMR confirmed $55 \mathrm{wt} \% \mathrm{NH}_{4} \mathrm{Cl}$ present. Mass Recovery: $386 \mathrm{mg}$. Allowing the ethanolic solution to stand promotes precipitation of $\mathrm{NH}_{4} \mathrm{Cl}$ which can be removed easily by filtration.

$1 \mathrm{H}-\mathrm{NMR}$ assay yield: $99 \%(0.172 \mathrm{~g} 1.00 \mathrm{mmol})$

Chemical Formula: $\mathrm{C}_{8} \mathrm{H}_{10} \mathrm{CINO}$

Molecular Weight: $171.62 \mathrm{gmol}^{-1}$

${ }^{1} \mathrm{H}$ NMR $\left(500 \mathrm{MHz}\right.$, DMSO- $\left.d_{6}\right) \delta=8.49(\mathrm{~s}, 3 \mathrm{H}), 8.02(\mathrm{~d}, \mathrm{~J}=7.7 \mathrm{~Hz}, 2 \mathrm{H}), 7.73(\mathrm{t}, J=7.4$ $\mathrm{Hz}, 1 \mathrm{H}), 7.59(\mathrm{t}, J=7.7 \mathrm{~Hz}, 2 \mathrm{H}), 4.58(\mathrm{q}, J=5.3 \mathrm{~Hz}, 2 \mathrm{H})$.

${ }^{1} \mathrm{H}$ NMR $\left(500 \mathrm{MHz}, \mathrm{D}_{2} \mathrm{O}\right) \delta=8.03-7.99(\mathrm{~m}, 2 \mathrm{H}), 7.76(\mathrm{t}, J=7.5 \mathrm{~Hz}, 1 \mathrm{H}), 7.60(\mathrm{t}, J=$ $7.5 \mathrm{~Hz}, 2 \mathrm{H}), 4.70(\mathrm{~s}, 2 \mathrm{H})$.

${ }^{13} \mathrm{C}$ NMR $\left(126 \mathrm{MHz}, \mathrm{DMSO}-d_{6}\right) \delta=193.5,135.0,134.1,129.5,128.7,45.4$.

${ }^{1} \mathrm{H}-\mathrm{NMR}$ and ${ }^{13} \mathrm{C}-\mathrm{NMR}$ were in agreement with the literature. ${ }^{18}$

2-amino-1-phenylethan-1-one hydrochloride $\mathbf{3 0}$ was also prepared as follows from Gabriel reaction product 31: To a stirred suspension of $\mathbf{3 1}(265 \mathrm{mg}, 1.00 \mathrm{mmol}$ ) in ethanol (10 $\mathrm{mL}$ ) was added hydrazine hydrate $(100 \mu \mathrm{L}, 2.00 \mathrm{mmol})$. The reaction mixture solubilised after 1 minute. The mixture was heated to $60^{\circ} \mathrm{C}$ and a colour change from a colourless clear solution to an orange solution was observed. After $2 \mathrm{~h}$ the reaction mixture was cooled to room temperature and solvent was removed in vacuo. The resulting orange crude material was acidified to $\mathrm{pH} 1$ using hydrochloric acid $(2 \mathrm{~N}, 5.0 \mathrm{~mL})$. The acidified suspension was then heated to $50{ }^{\circ} \mathrm{C}$ for $0.5 \mathrm{~h}$ to hydrolyse any hydrazone present. A brown precipitate was observed forming. After 30 minutes the reaction mixture was cooled to room temperature and the solid removed by filtration. Water was removed from the filtrate in vacuo to give a brown solid which was triturated with acetonitrile $(1 \times 30 \mathrm{~mL})$ and TBME $(2 \times 30 \mathrm{~mL})$ to give a pale yellow solid. Yield: $83 \%(142 \mathrm{mg}, 0.83 \mathrm{mmol})$. 
${ }^{1} \mathrm{H}$ NMR $\left(500 \mathrm{MHz}, \mathrm{DMSO}-d_{6}\right)$

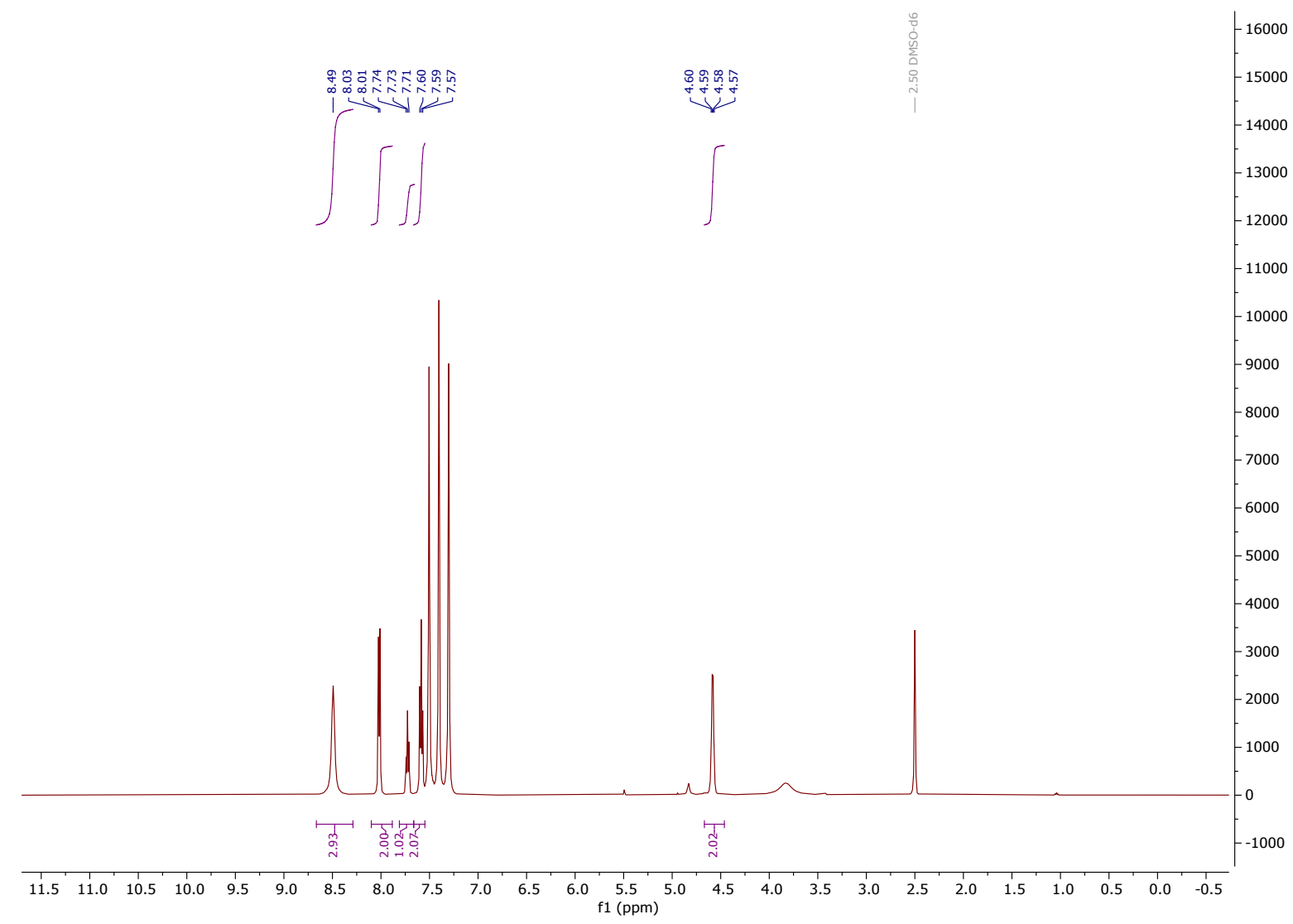

${ }^{13} \mathrm{C}$ NMR (126 MHz, DMSO- $d_{6}$ )

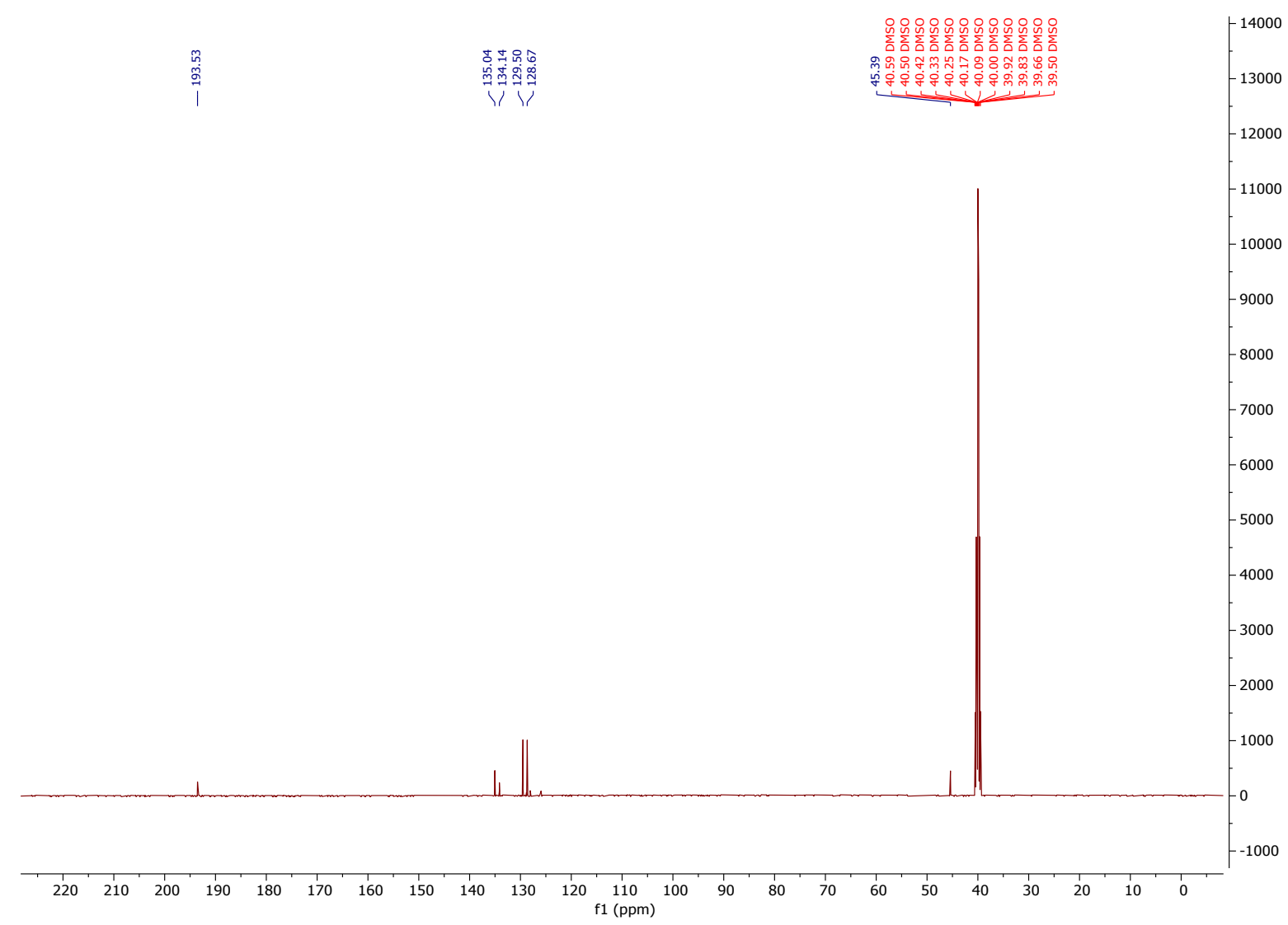


${ }^{1} \mathrm{H}$ NMR $\left(500 \mathrm{MHz}, \mathrm{D}_{2} \mathrm{O}\right)$

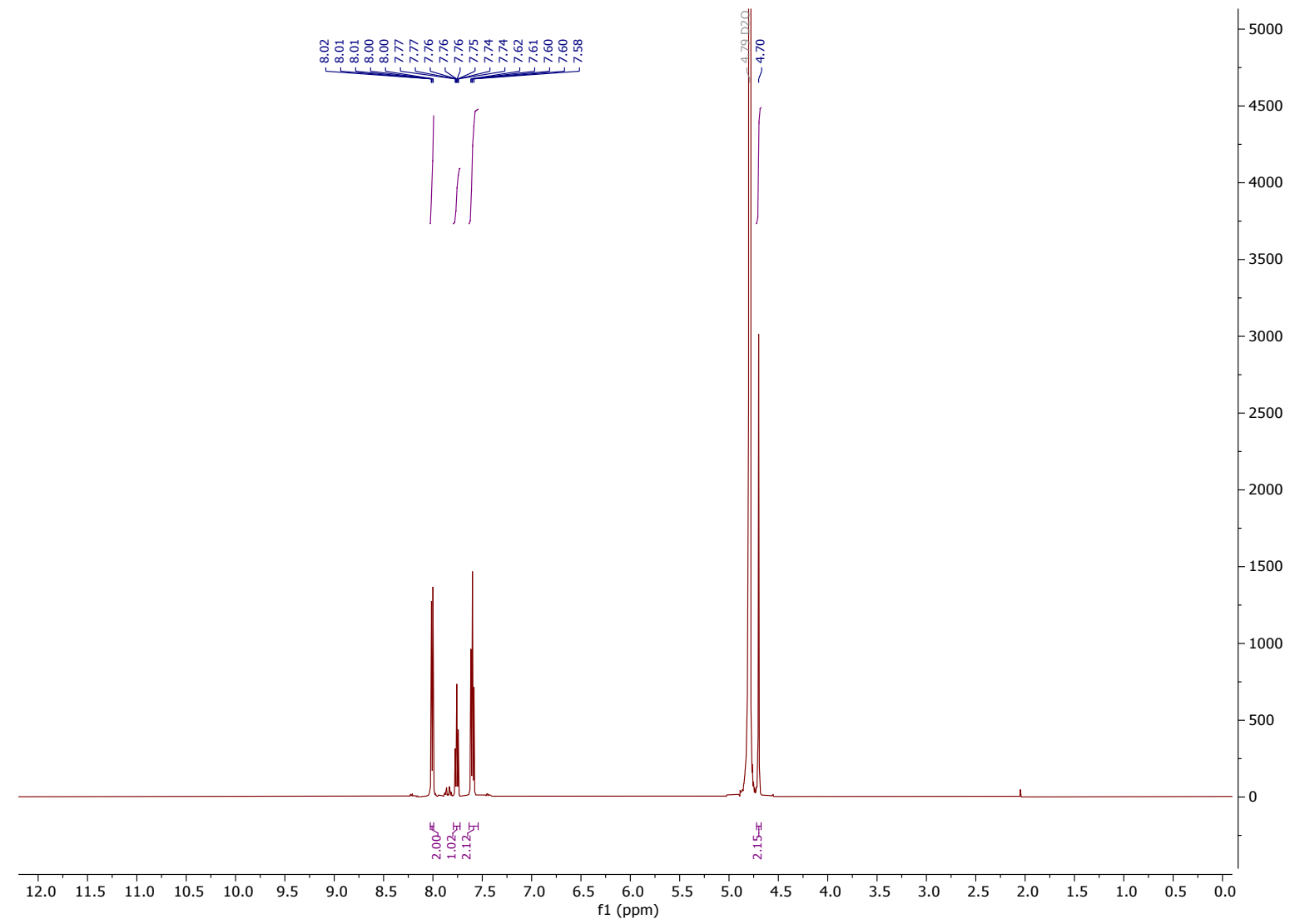

2-(2-Oxo-2-phenylethyl)isoindoline-1,3-dione 31<smiles>O=C(CN1C(=O)c2ccccc2C1=O)c1ccccc1</smiles>

To a stirred solution of potassium phthalimide (370 mg, $2.00 \mathrm{mmol}$ ) in $\mathrm{N}, \mathrm{N}$ dimethylformamide $(25 \mathrm{~mL}$ ) was added 2-bromoacetophenone (398 $\mathrm{mg}, 2.00 \mathrm{mmol})$. The reaction mixture was stirred at $70{ }^{\circ} \mathrm{C}$ for three $\mathrm{h}$ and then allowed to cool to room temperature. The reaction mixture was poured into deionised water $(80 \mathrm{~mL})$ and stirred at room temperature. A white precipitate formed immediately. The resulting suspension was stirred for 30 minutes before the white solid was isolated by vacuum filtration. The solid was washed with deionised water $(2 \times 10 \mathrm{~mL})$ and dried under high vacuum to give the title product as a white solid in $81 \%$ yield $(430 \mathrm{mg}, 1.62 \mathrm{mmol})$

Chemical Formula: $\mathrm{C}_{16} \mathrm{H}_{11} \mathrm{NO}_{3}$

Molecular Weight: $265.27 \mathrm{gmol}^{-1}$

Appearance: White Solid

Yield: $81 \%(430 \mathrm{mg}, 1.62 \mathrm{mmol})$ 
${ }^{1} \mathrm{H}$ NMR $\left(500 \mathrm{MHz}, \mathrm{CDCl}_{3}\right) \delta=8.04-8.00(\mathrm{~m}, 2 \mathrm{H}), 7.91(\mathrm{dd}, J=5.4,3.1 \mathrm{~Hz}, 2 \mathrm{H}), 7.76$ $(\mathrm{dd}, J=5.4,3.1 \mathrm{~Hz}, 2 \mathrm{H}), 7.67-7.62(\mathrm{~m}, 1 \mathrm{H}), 7.53(\mathrm{t}, J=7.8 \mathrm{~Hz}, 2 \mathrm{H}), 5.14(\mathrm{~s}, 2 \mathrm{H})$.

${ }^{13} \mathrm{C}$ NMR $\left(126 \mathrm{MHz}_{1} \mathrm{CDCl}_{3}\right) \delta=191.0,167.9,134.4,134.1,134.1,132.3,128.9,128.2$, $123.6,44.2$.

${ }^{1} \mathrm{H}$ and ${ }^{13} \mathrm{C}$ NMR were in agreement with the literature. ${ }^{19}$

${ }^{1} \mathrm{H}$ NMR $\left(500 \mathrm{MHz}, \mathrm{CDCl}_{3}\right)$

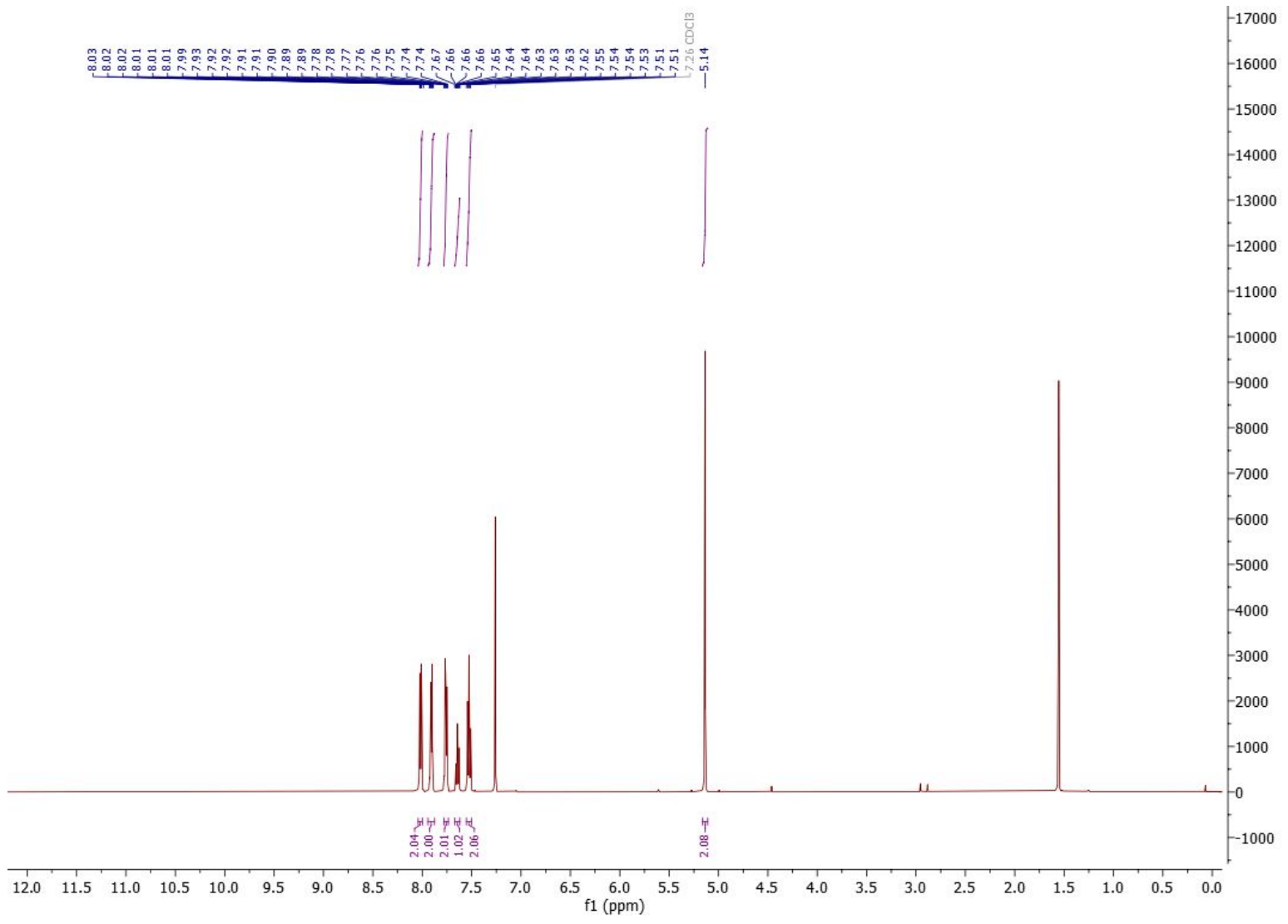




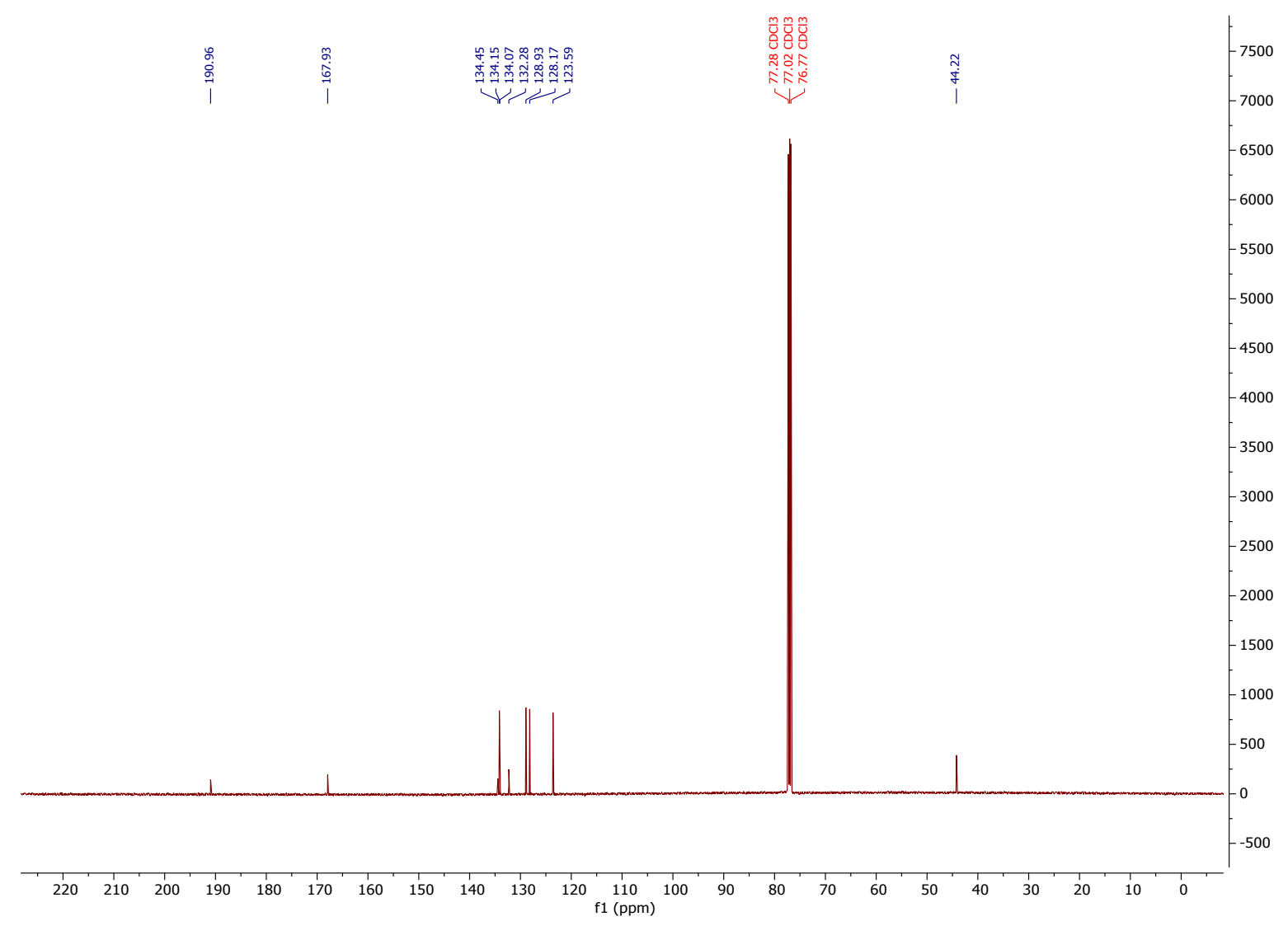

Gabriel Deprotection - Hydrazinolysis

2-Amino-1-phenylethan-1-one hydrochloride $\mathbf{3 0}$<smiles>NCC(=O)c1ccccc1</smiles>

Chemical Formula: $\mathrm{C}_{8} \mathrm{H}_{10} \mathrm{CINO}$

Molecular Weight: $171.62 \mathrm{gmol}^{-1}$

${ }^{1} \mathrm{H}$ NMR $\left(500 \mathrm{MHz}, \mathrm{DMSO}-d_{6}\right) \delta=8.49(\mathrm{~s}, 3 \mathrm{H}), 8.02(\mathrm{~d}, \mathrm{~J}=7.7 \mathrm{~Hz}, 2 \mathrm{H}), 7.73(\mathrm{t}, J=7.4$ $\mathrm{Hz}, 1 \mathrm{H}), 7.59(\mathrm{t}, J=7.7 \mathrm{~Hz}, 2 \mathrm{H}), 4.58(\mathrm{q}, J=5.3 \mathrm{~Hz}, 2 \mathrm{H})$.

${ }^{1} \mathrm{H}$ NMR $\left(500 \mathrm{MHz}, \mathrm{D}_{2} \mathrm{O}\right) \delta=8.03-7.99(\mathrm{~m}, 2 \mathrm{H}), 7.76(\mathrm{t}, J=7.5 \mathrm{~Hz}, 1 \mathrm{H}), 7.60(\mathrm{t}, J=$ $7.5 \mathrm{~Hz}, 2 \mathrm{H}), 4.70(\mathrm{~s}, 2 \mathrm{H})$.

${ }^{13} \mathrm{C}$ NMR $\left(126 \mathrm{MHz}\right.$, DMSO- $\left.d_{6}\right) \delta=193.5,135.0,134.1,129.5,128.7,45.4$.

${ }^{1} \mathrm{H}-\mathrm{NMR}$ and ${ }^{13} \mathrm{C}-\mathrm{NMR}$ were in agreement with the literature. ${ }^{18}$

2-amino-1-phenylethan-1-one hydrochloride $\mathbf{3 0}$ was also prepared as follows from Gabriel reaction product 31: To a stirred suspension of $\mathbf{3 1}(265 \mathrm{mg}, 1.00 \mathrm{mmol})$ in ethanol (10 $\mathrm{mL}$ ) was added hydrazine hydrate $(100 \mu \mathrm{L}, 2.00 \mathrm{mmol})$. The reaction mixture solubilised after 1 minute. The mixture was heated to $60^{\circ} \mathrm{C}$ and a colour change from a colourless 
clear solution to an orange solution was observed. After $2 \mathrm{~h}$ the reaction mixture was cooled to room temperature and solvent was removed in vacuo. The resulting orange crude material was acidified to $\mathrm{pH} 1$ using hydrochloric acid $(2 \mathrm{~N}, 5.0 \mathrm{~mL})$. The acidified suspension was then heated to $50{ }^{\circ} \mathrm{C}$ for $0.5 \mathrm{~h}$ to hydrolyse any hydrazone present. A brown precipitate was observed forming. After 30 minutes the reaction mixture was cooled to room temperature and the solid removed by filtration. Water was removed from the filtrate in vacuo to give a brown solid which was triturated with acetonitrile $(1 \times 30 \mathrm{~mL})$ and TBME $(2 \times 30 \mathrm{~mL})$ to give a pale yellow solid. Yield: $83 \%(142 \mathrm{mg}, 0.83 \mathrm{mmol})$.

\section{Differential Scanning Calorimetry}<smiles>BrC[N+]12CN3CN(CN(C3)C1)C2</smiles>

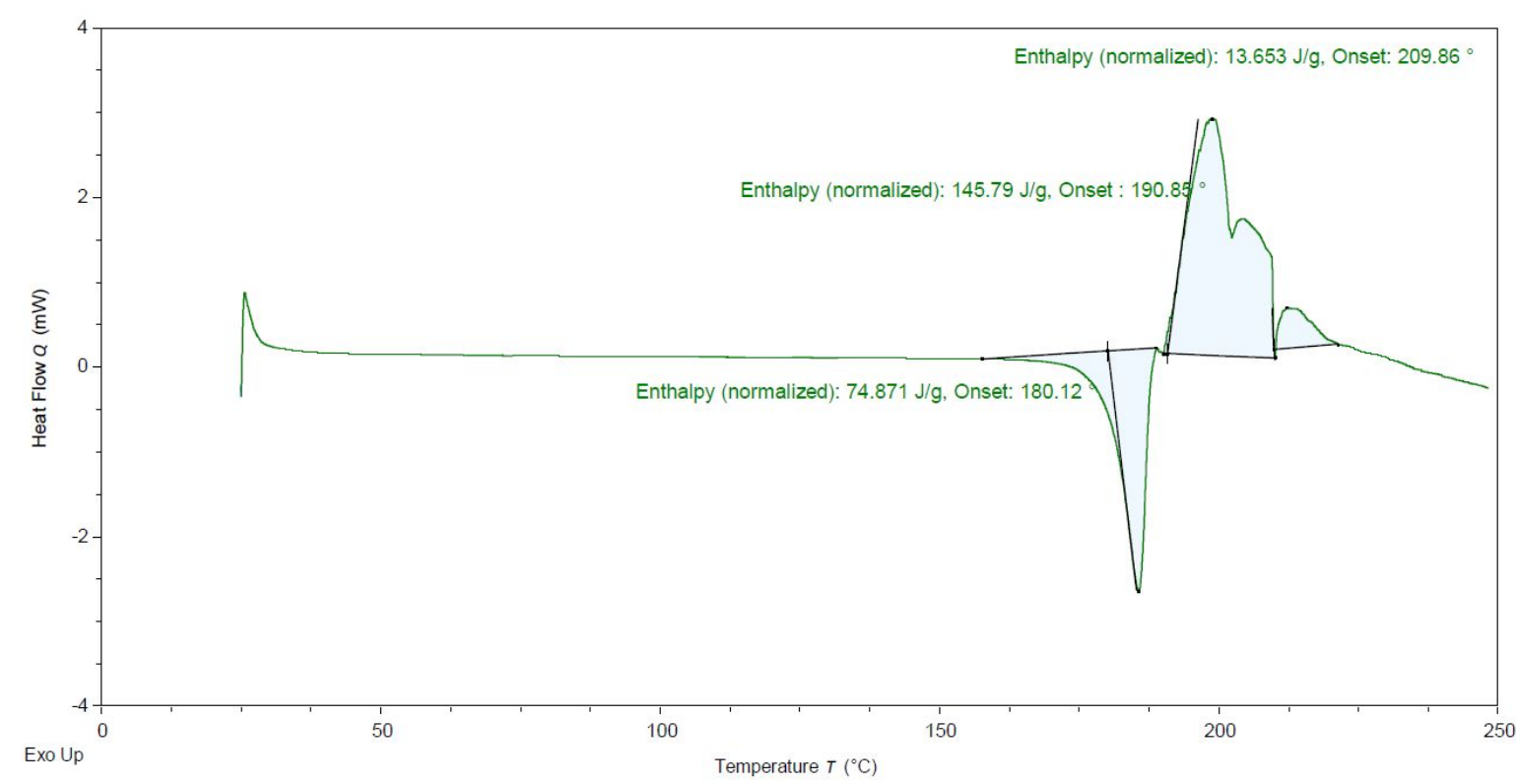


(N)

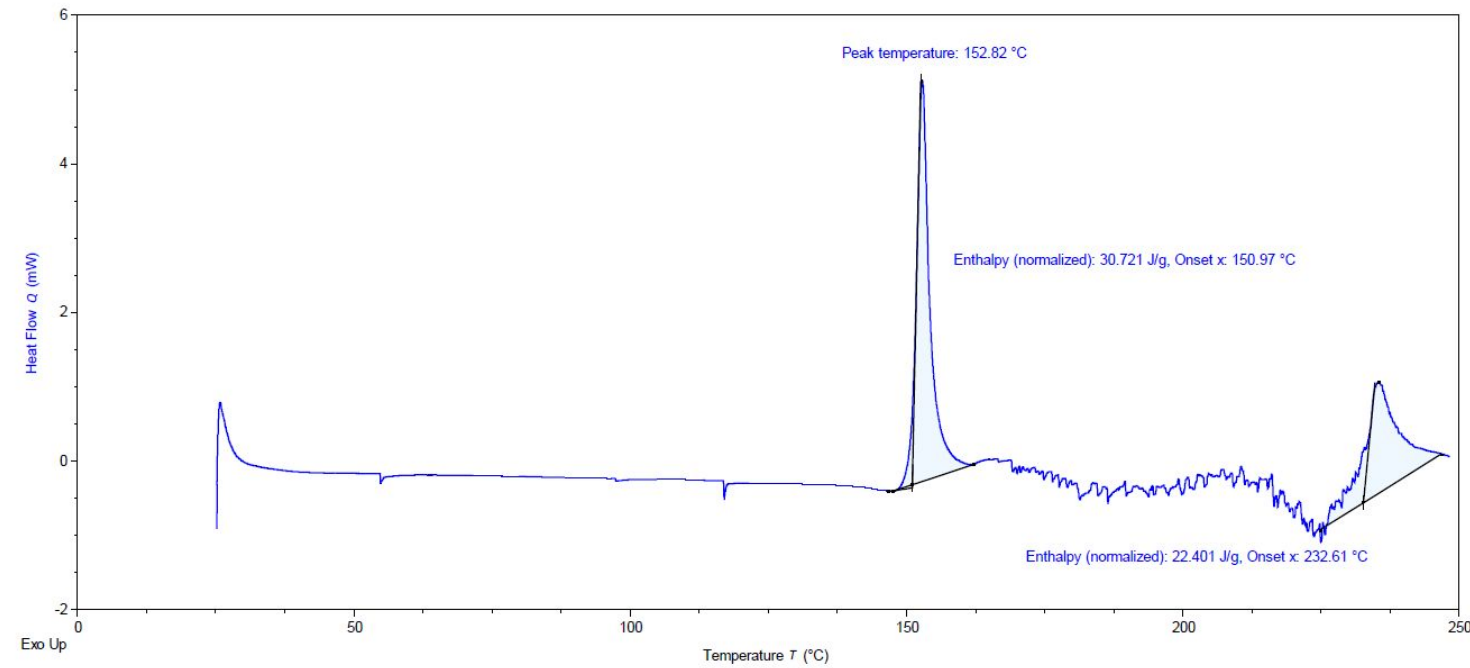

(n)

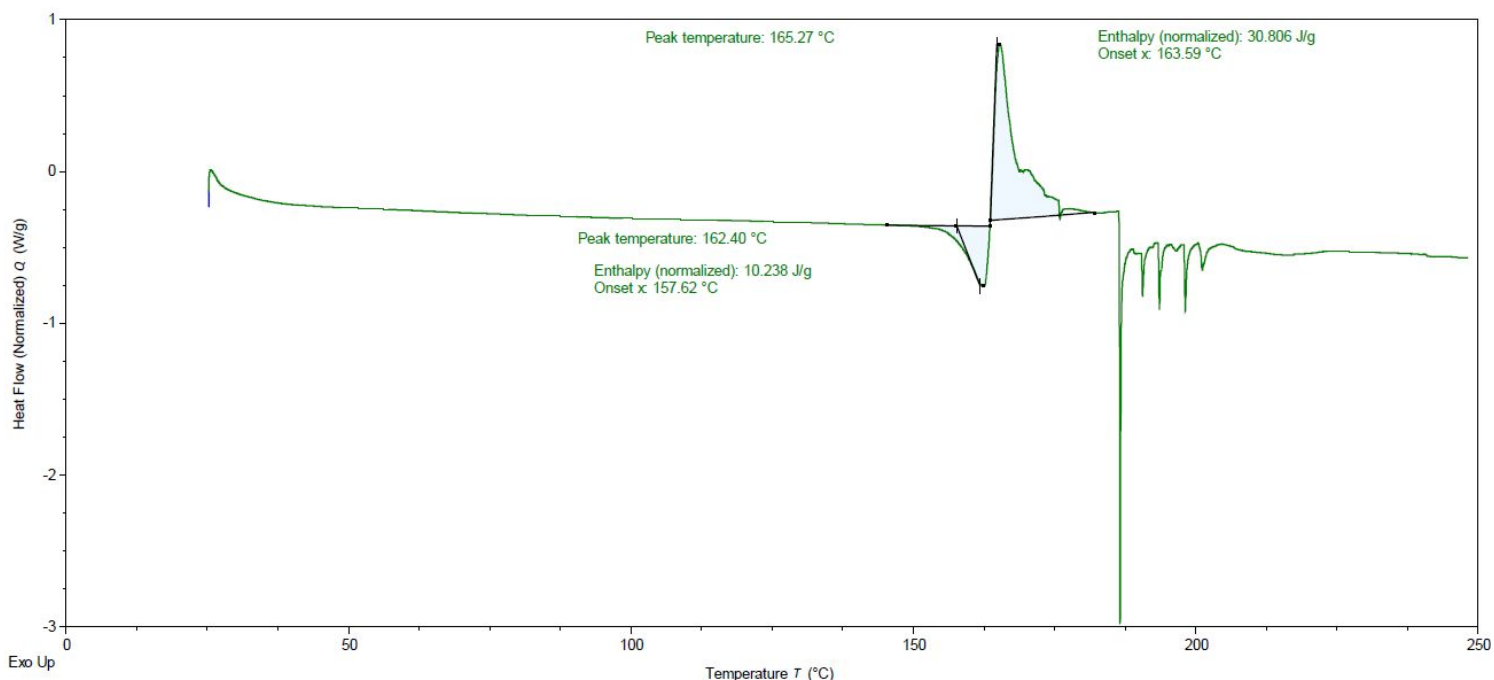



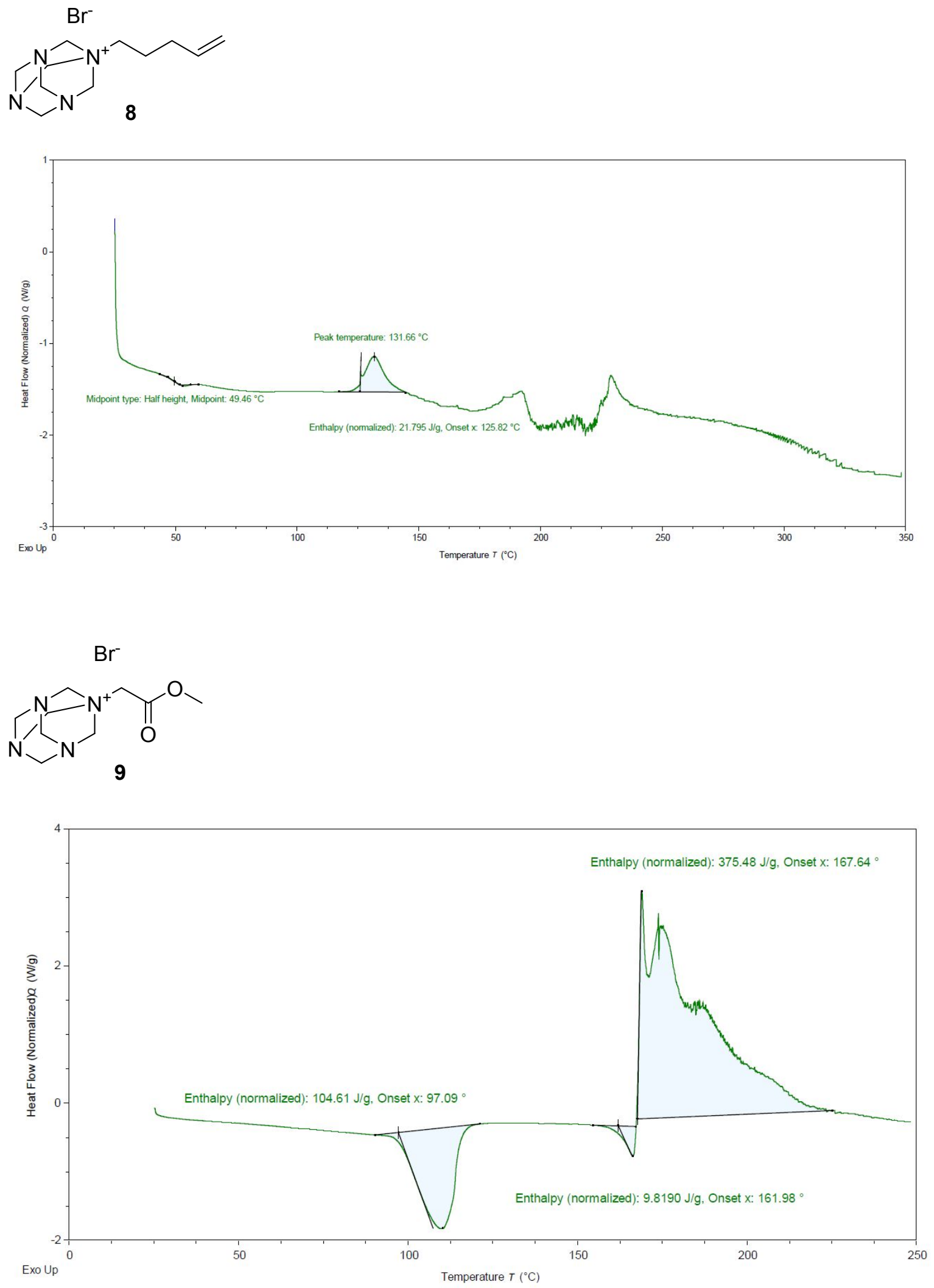

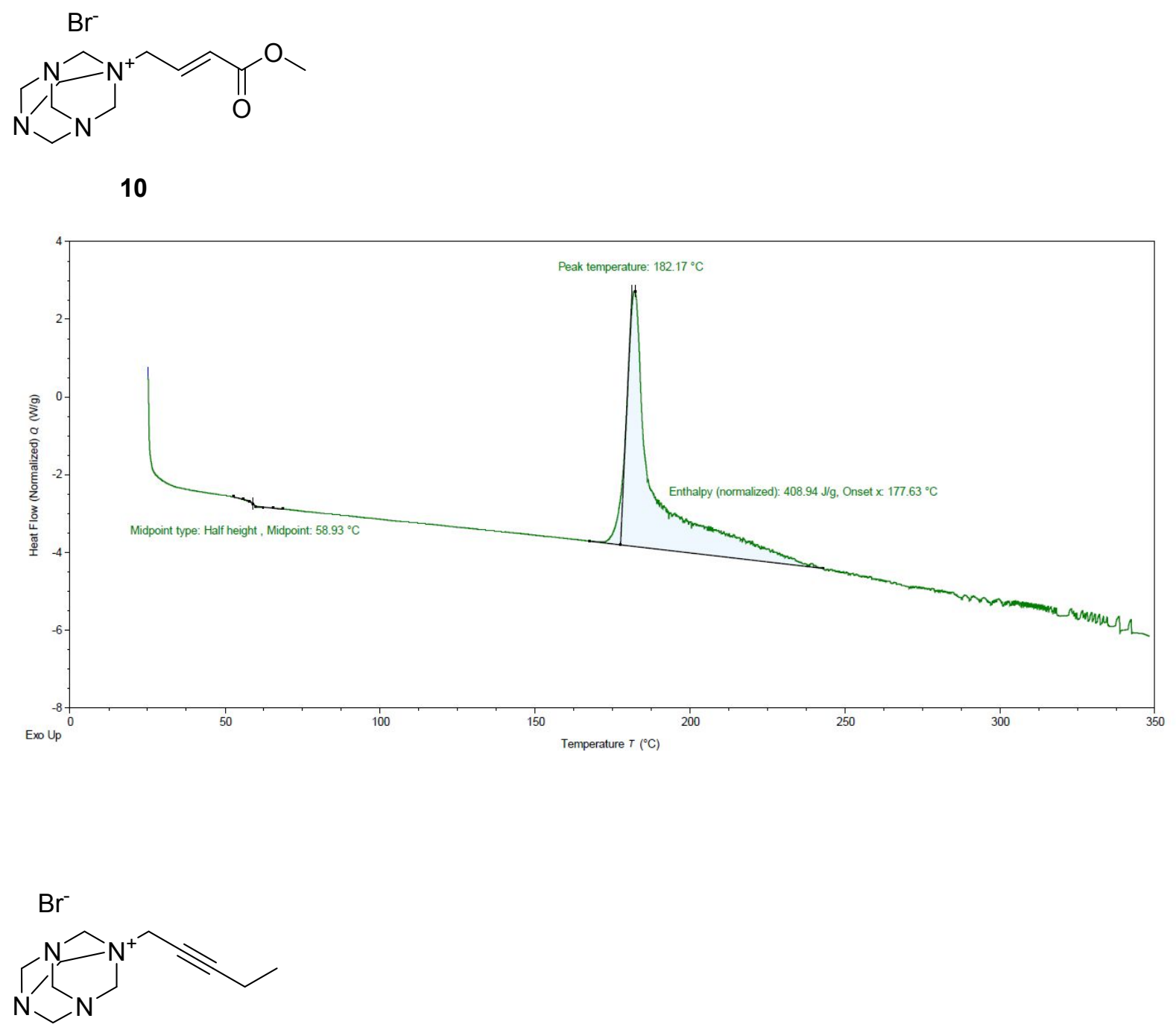

11

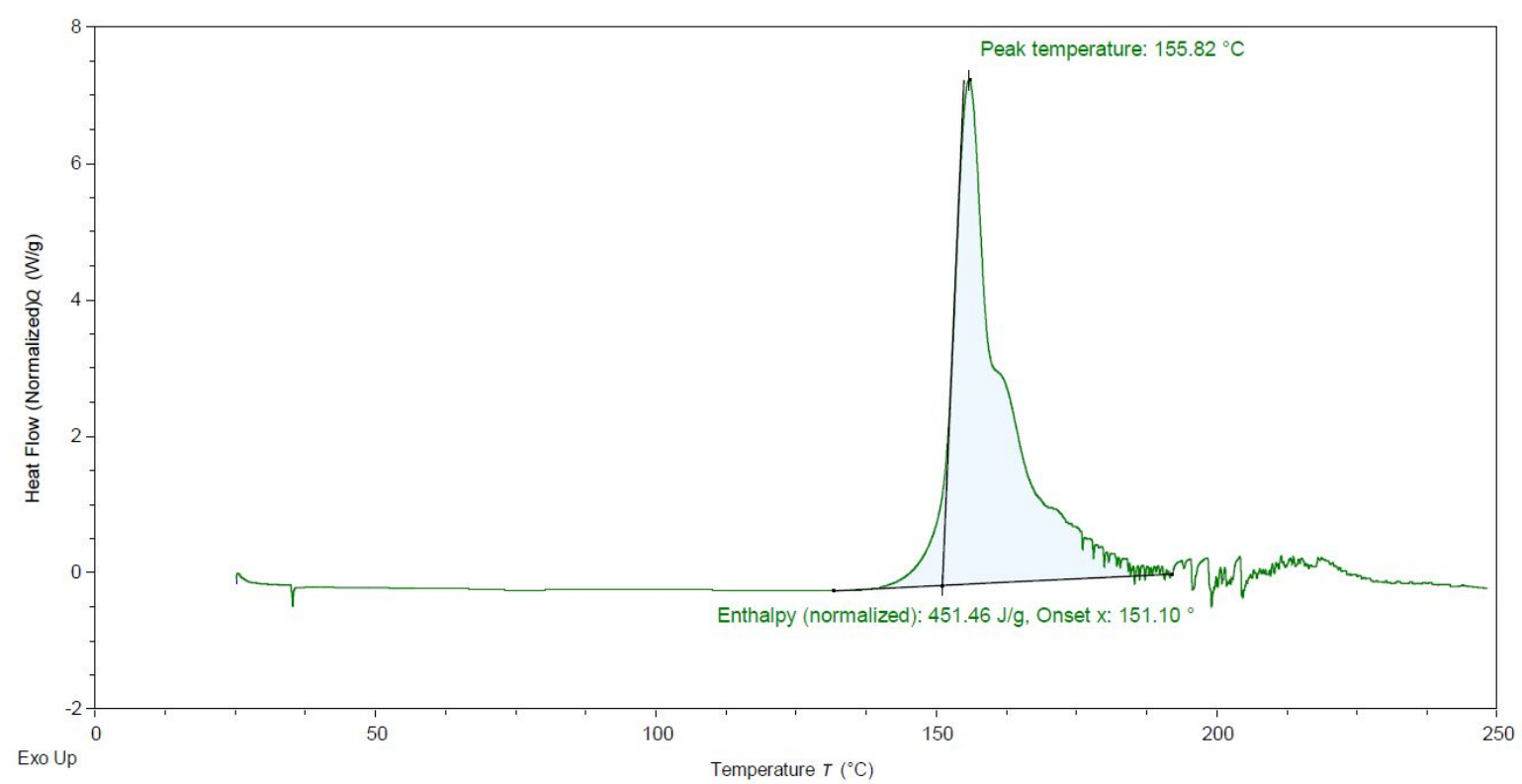

S59 
<smiles>C=C(C)C[N+]12CN3CN(CN(C3)C1)C2</smiles>

12
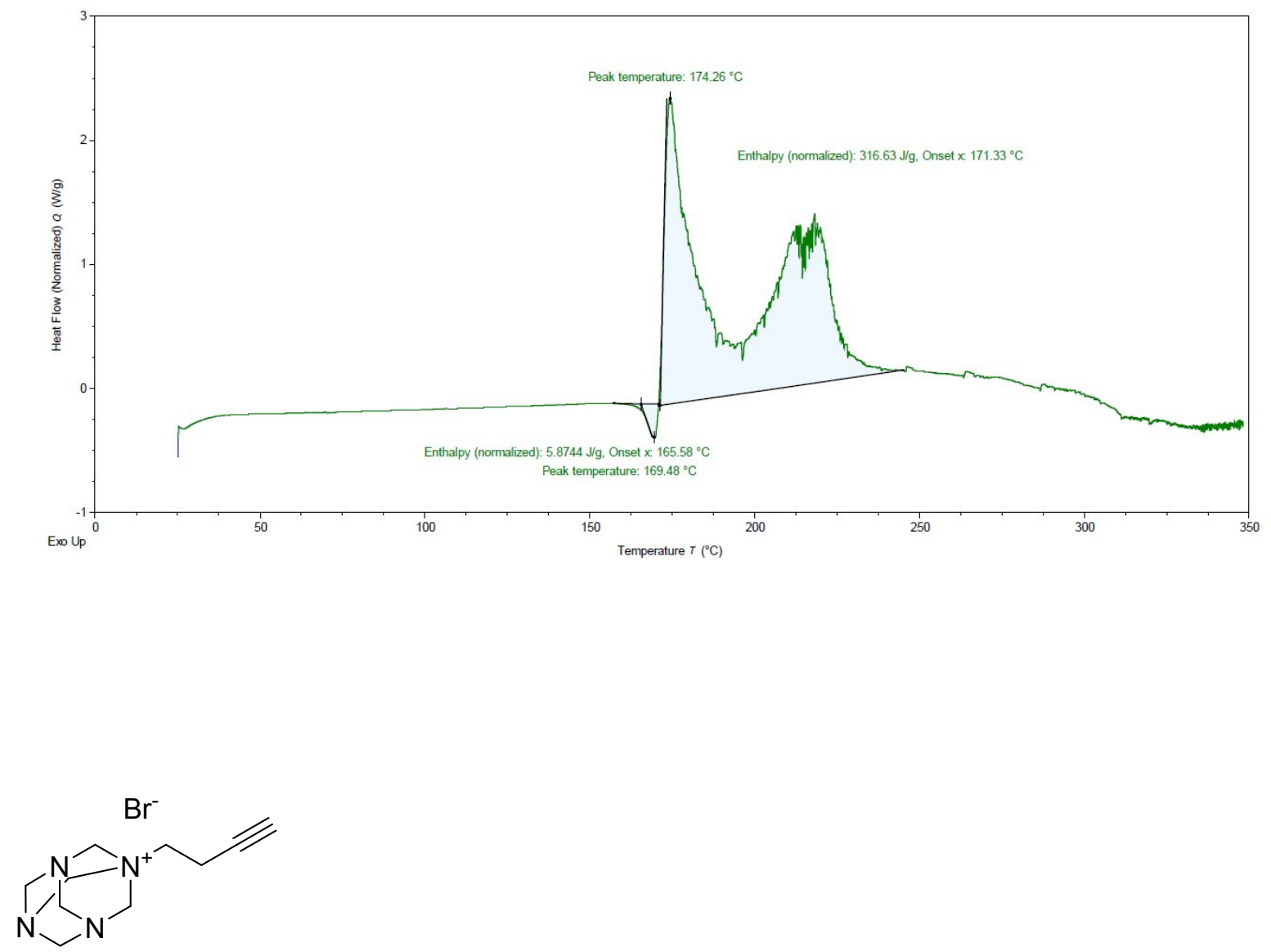

14

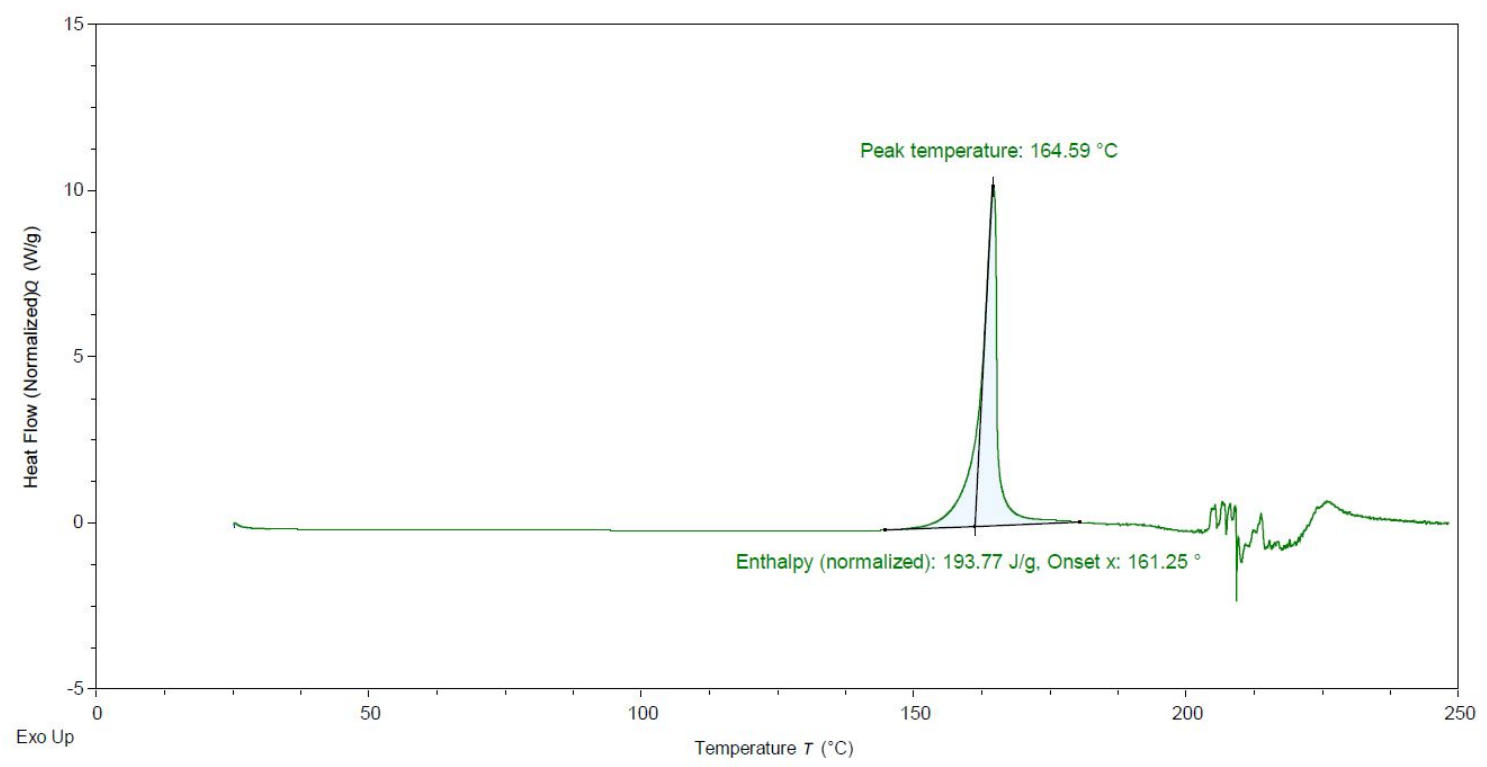

S60 
<smiles>O=C(C[N+]12CN3CN(CN(C3)C1)C2)c1ccccc1</smiles>

15
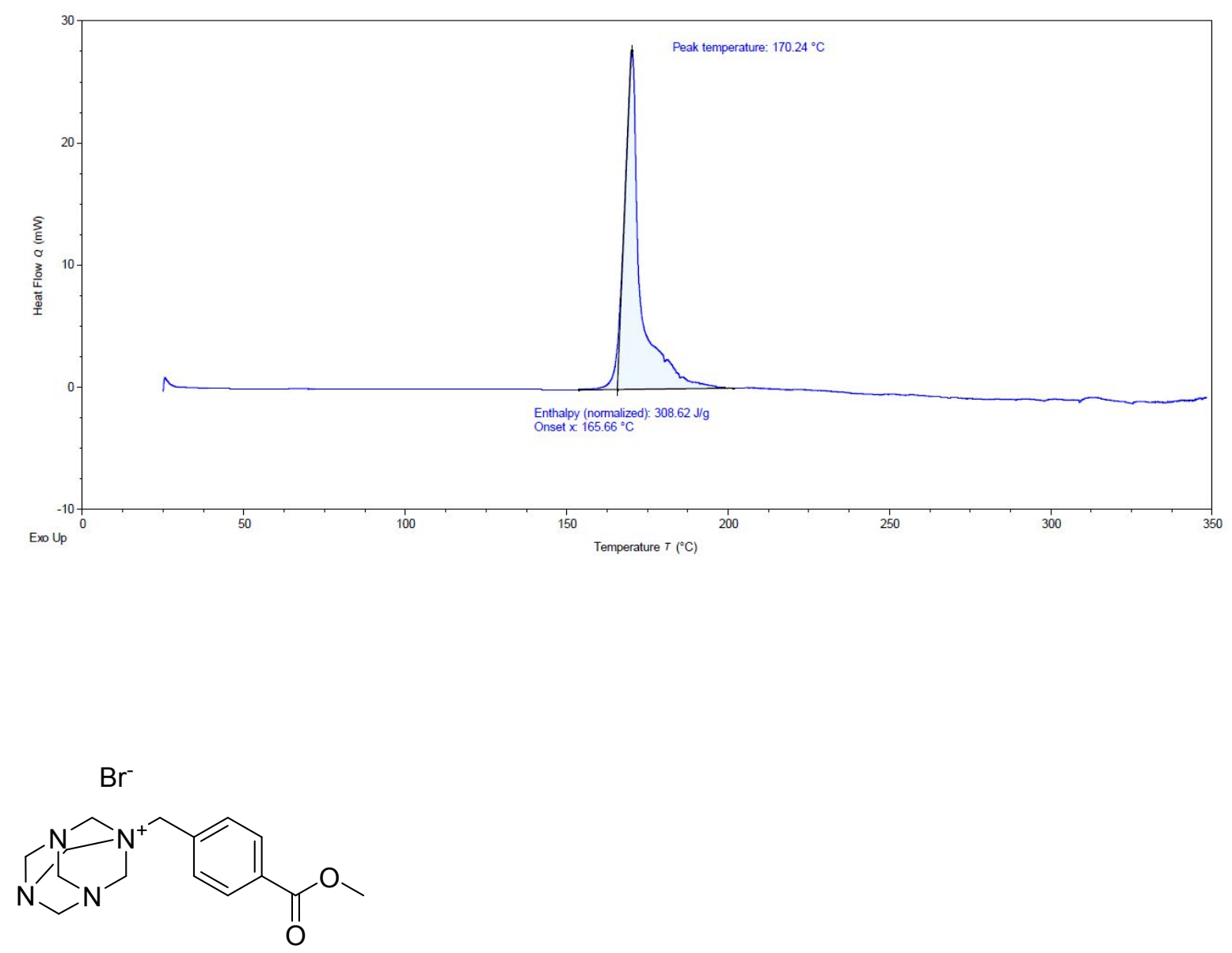

16

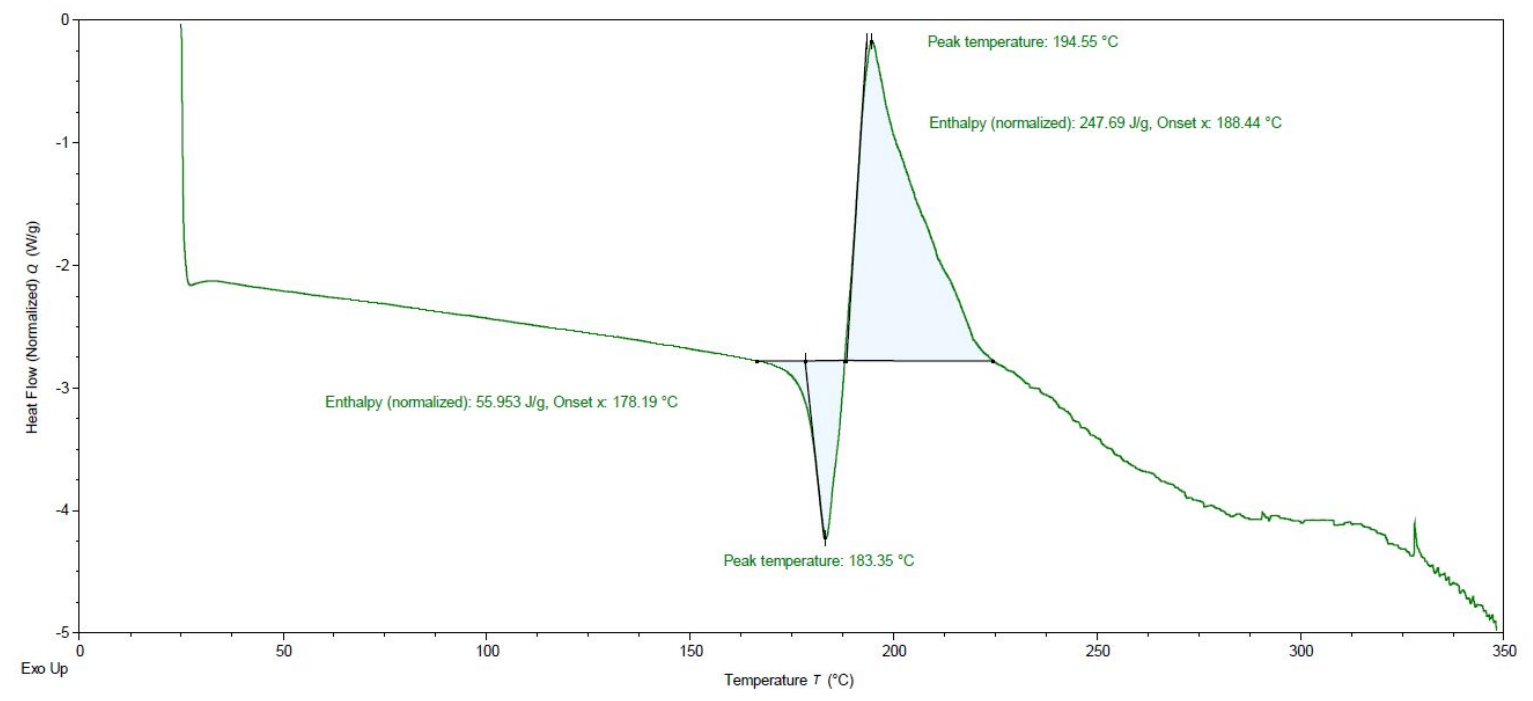

S61 
<smiles>COc1ccc(C(=O)C[N+]23CN4CN(CN(C4)C2)C3)cc1</smiles>

17

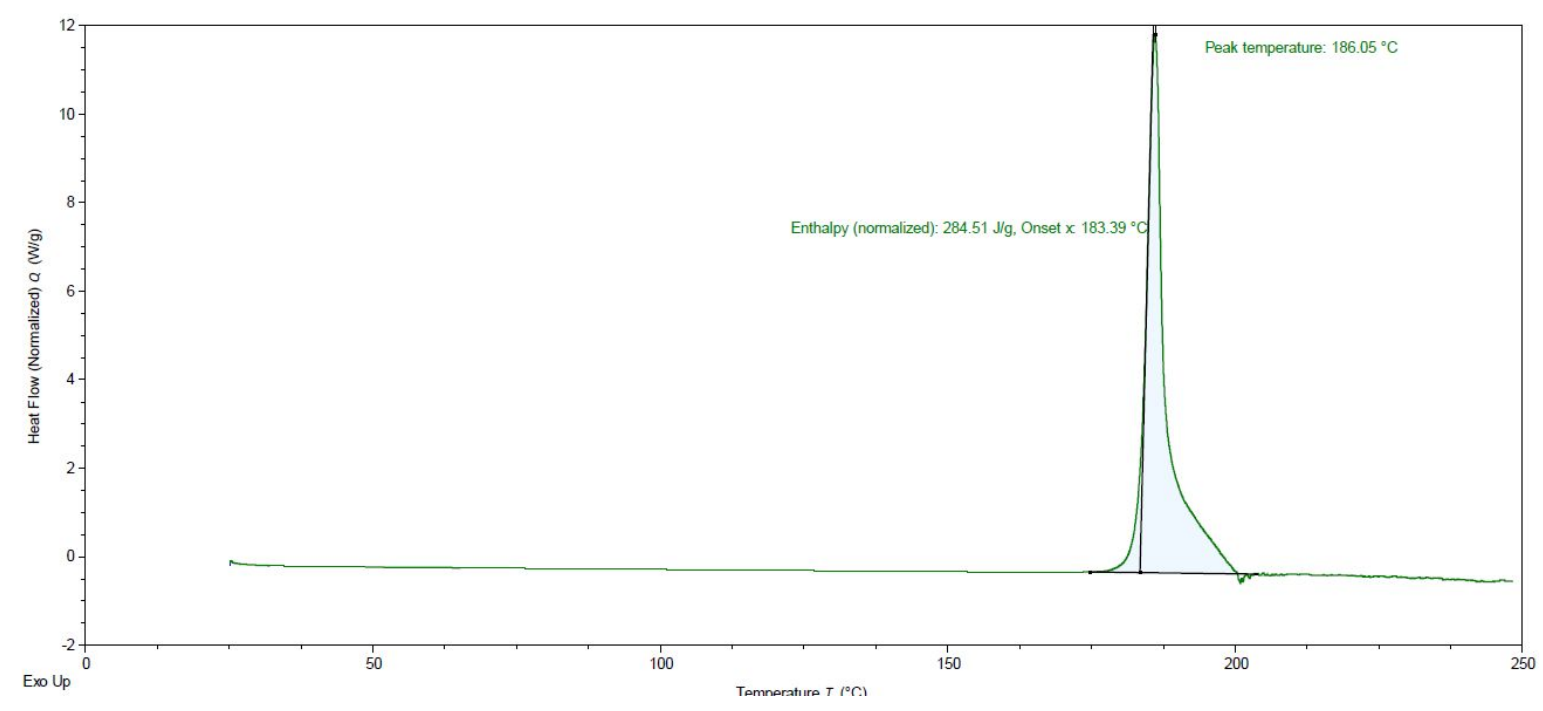

(18)

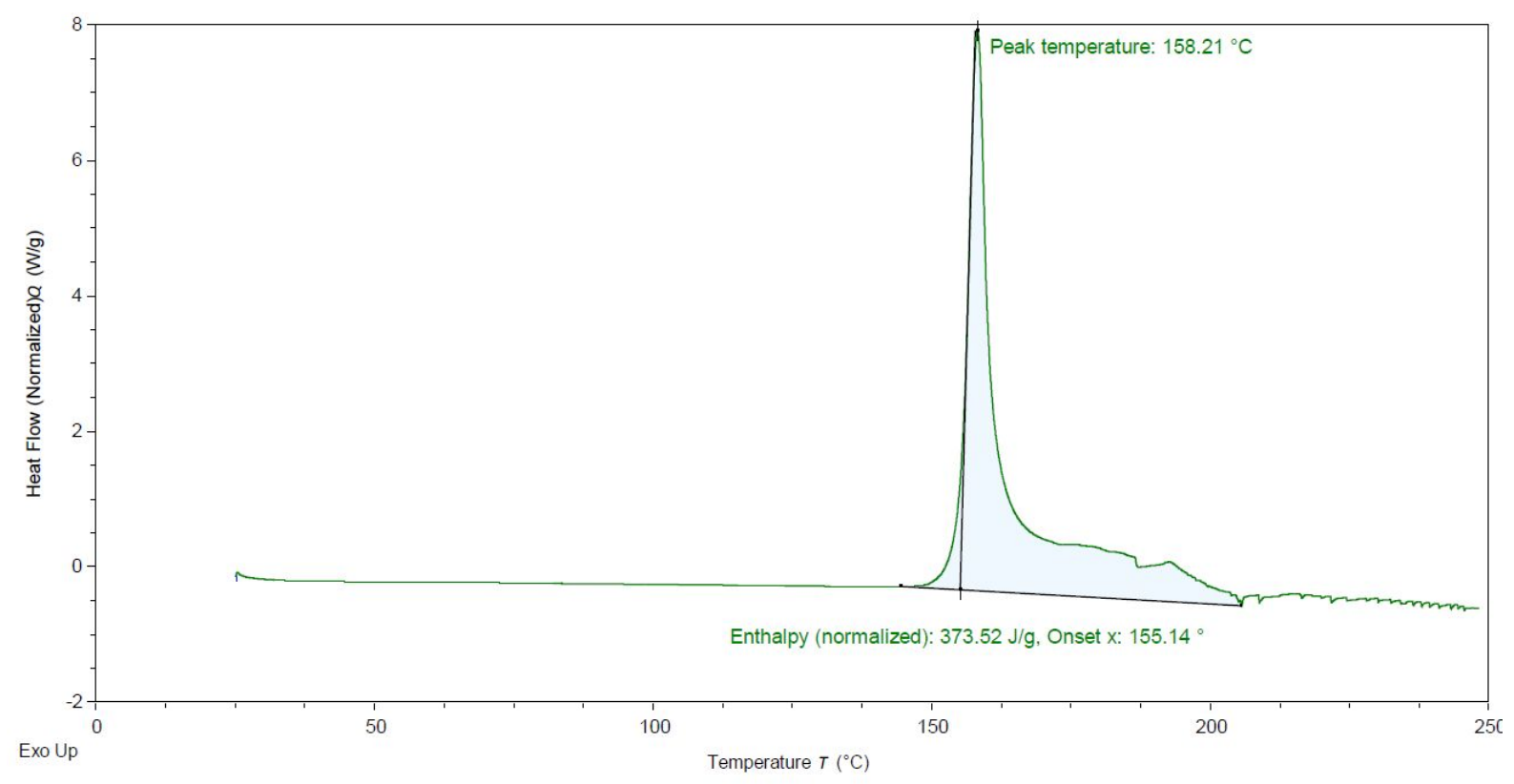


<smiles>O=C(C[N+]12CN3CN(CN(C3)C1)C2)c1cc2ccccc2o1</smiles>

19
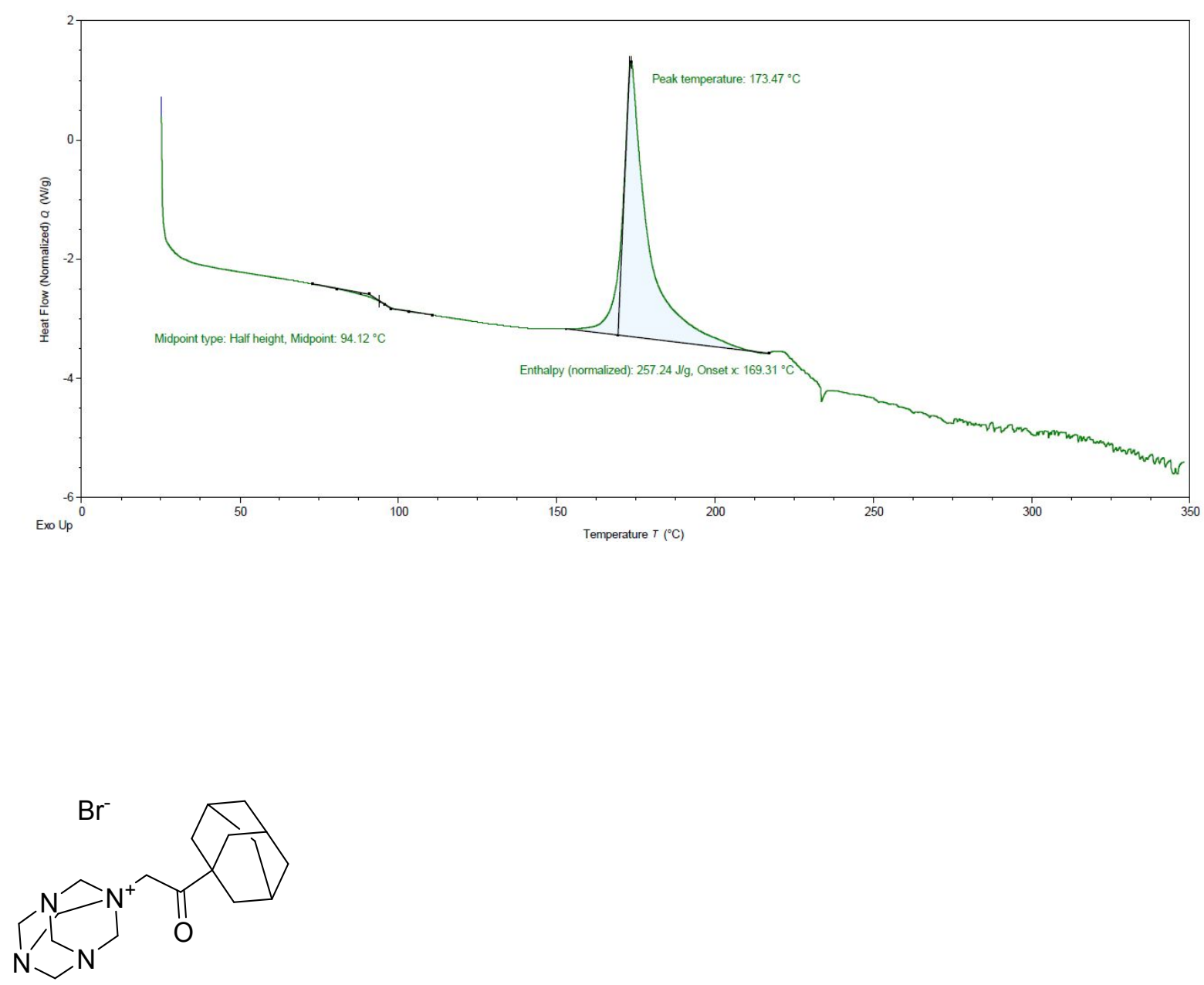

20

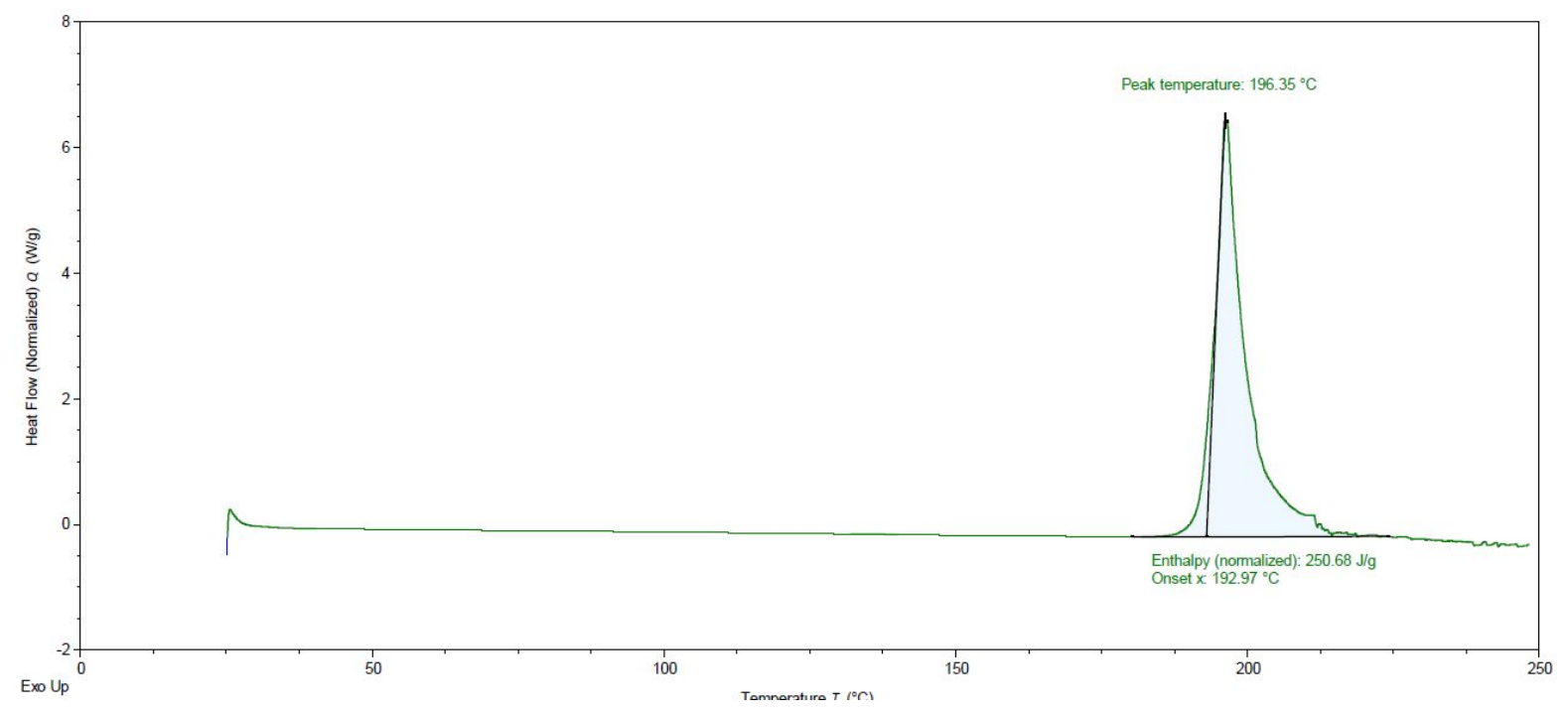

S63 
<smiles>COC(=O)c1ccc(C[N+]23CN4CN(CN(C4)C2)C3)nc1</smiles>

21
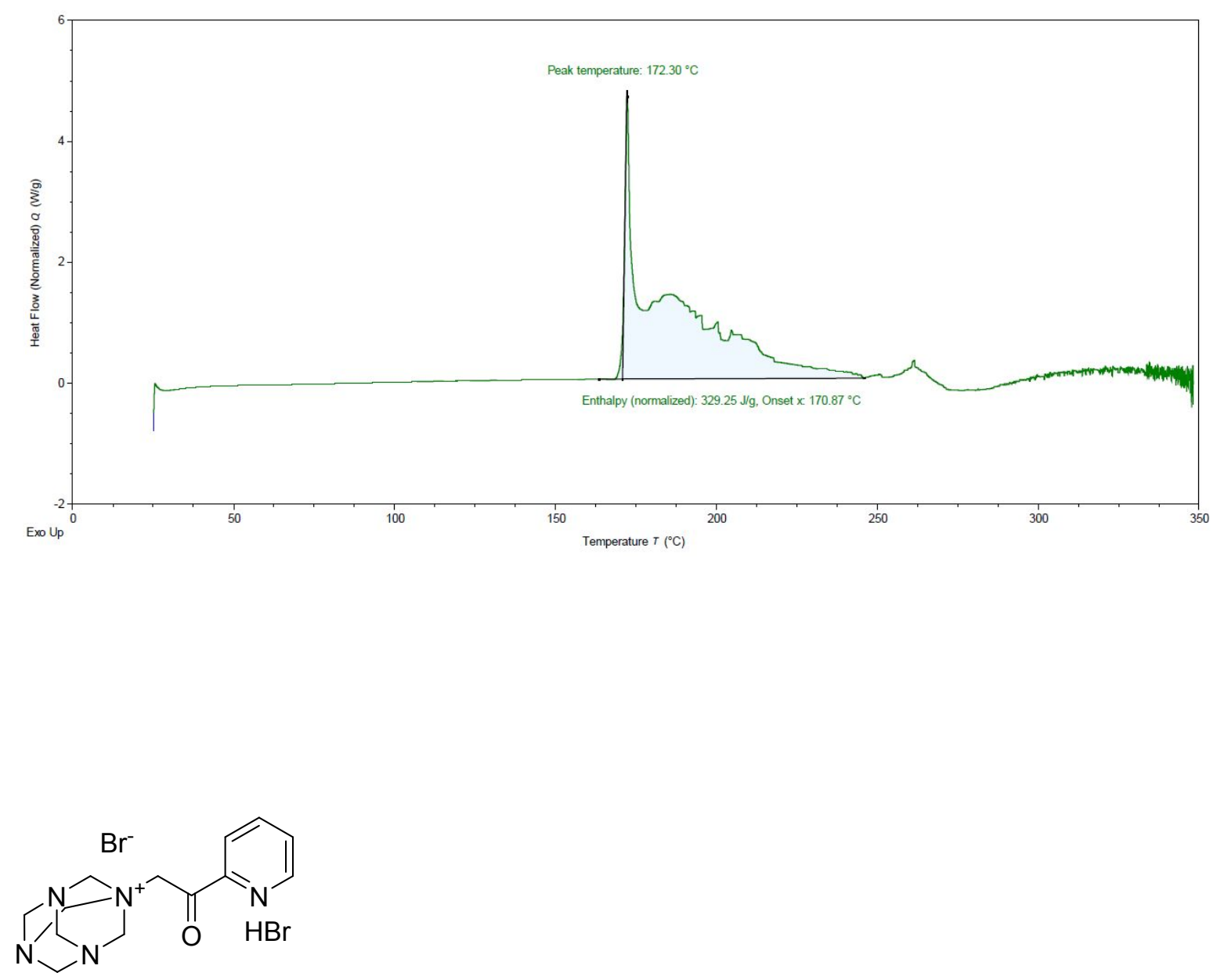

22

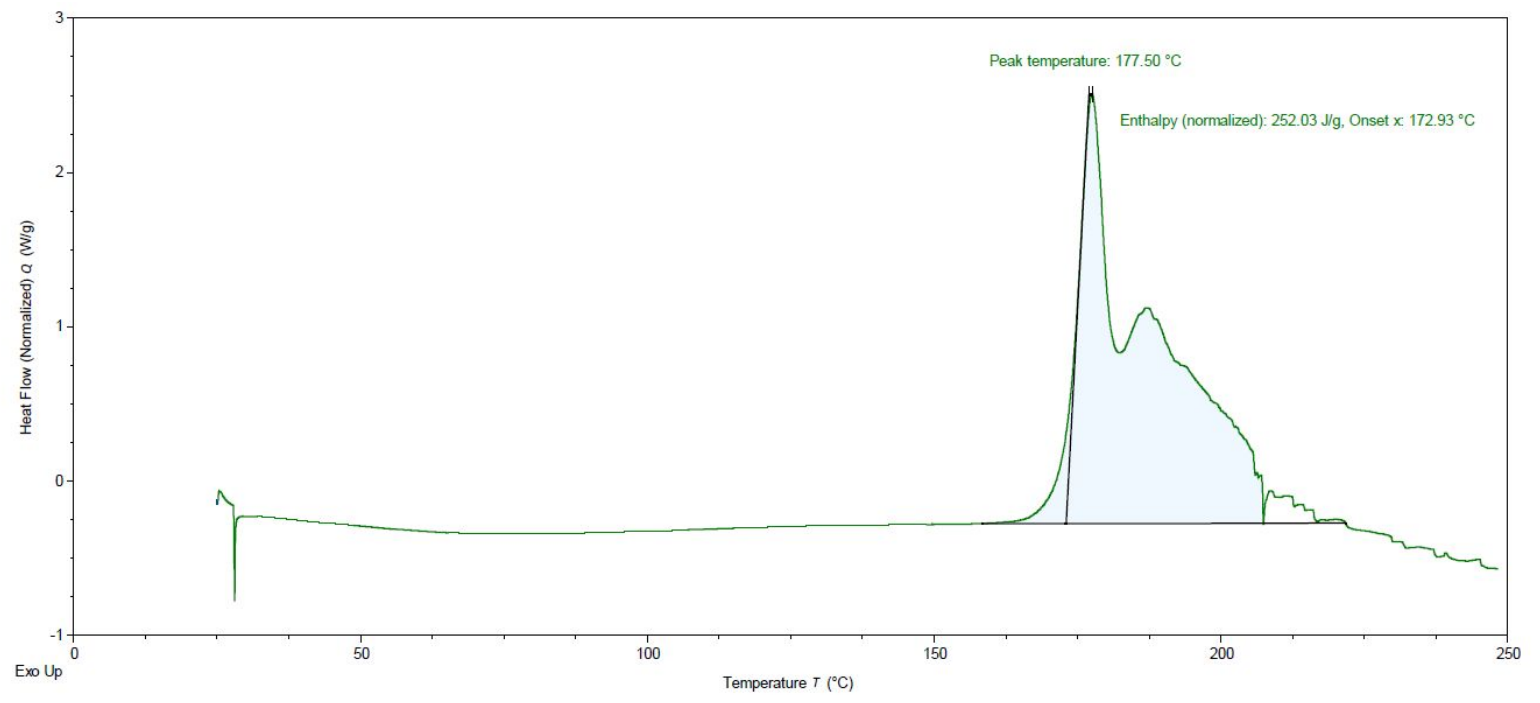

S64 
<smiles>CC(C)(C)OC(=O)N1CCC(C(=O)C[N+]23CCN4CN(CN(C4)C2)C3)CC1</smiles>

23

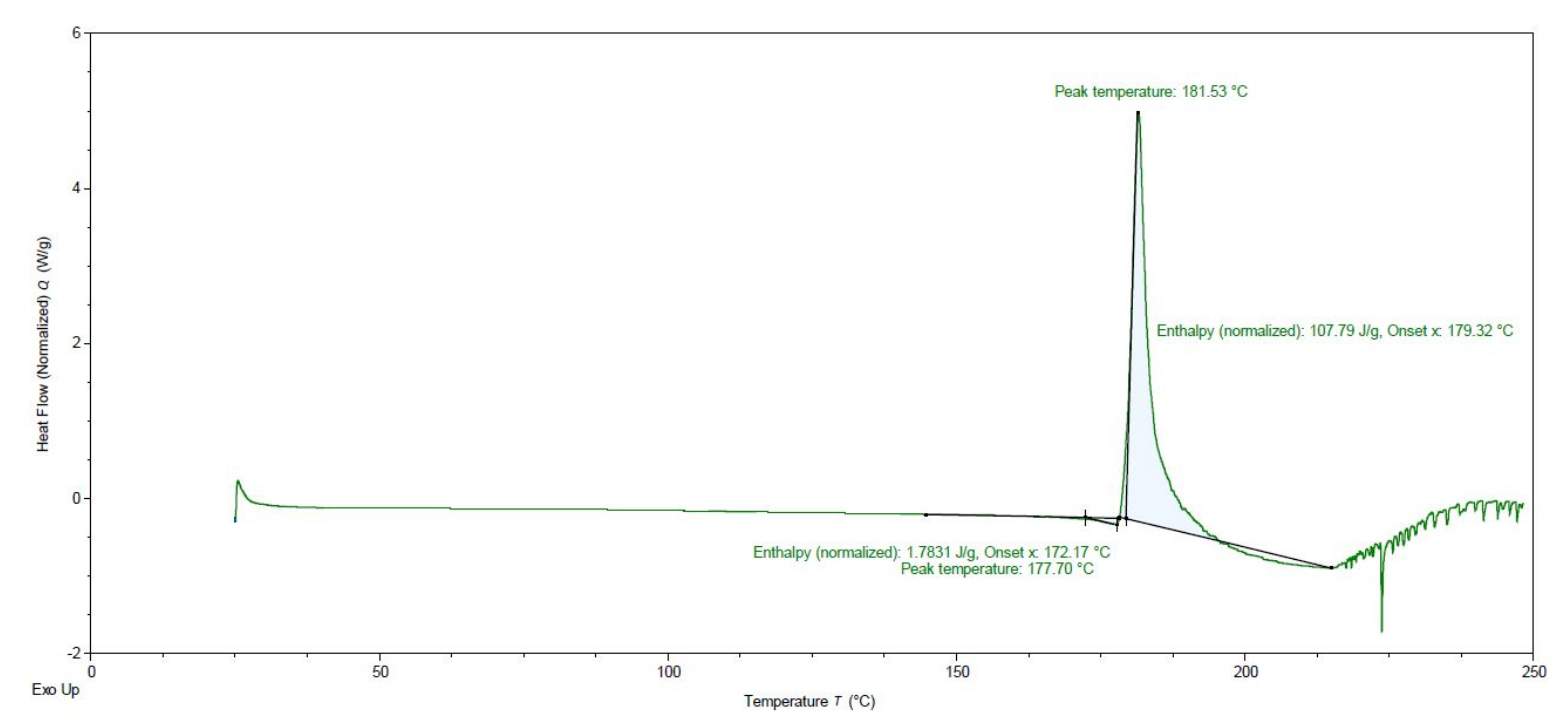

$\mathrm{Br}^{-}$

经

24

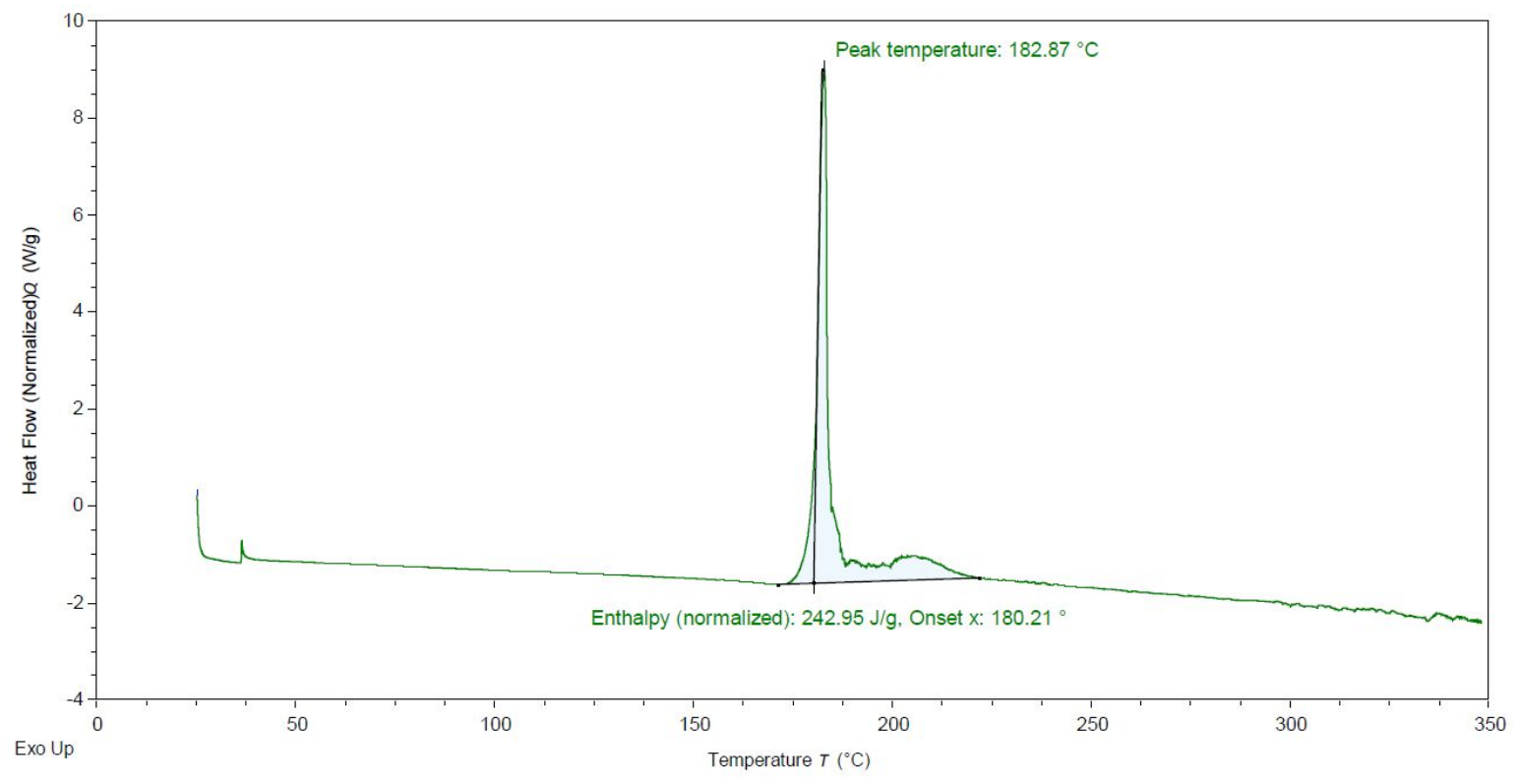




\section{References}

1. Levashova, V. I., Nitrogen-containing compounds for growth suppression of sulfate-reducing bacteria in petroleum production. Pet. Chem. 2002, 42, 132-135

2. Li, C.-B.; Huang, L.-S.; Wu, R.-S.; Xu, D.-Z., Bridged Alkyl Ionic Liquid-Catalyzed Tandem Reaction for Synthesis of Spiro[4H-pyran-3,3'-oxindoles] in Aqueous Ethanol Solution. ChemistrySelect 2019, 4 (5), 1635-163910.1002/slct.201803905.

3. Xue, Y.; Cui, Z.; Guo, W.; Zhao, H. Tetracationic quaternary ammonium salt containing hexamethylene tetraamine as skeleton and its preparation. CN 102702209, 03/10/2012, 2012.

4. Liu, Z. Preparation method of noradrenalin. CN 107298646, 27/10/2017, 2017.

5. Li, W.; Chen, L.; Gao, H.; Ran, Y. Method for synthesizing 3-chloro-4methoxybenzylamine. CN 105712893, 29/06/2016, 2016.

6. Ohnoda, M.; Uda, J.; Iwai, Y.; Tanase, T. Preparation of (4-methoxy-3alkoxycarbonylphenylmethyl)hexamethylenetetraminium chlorides as intermediates for antidiabetics and antihyperlipidemics. 13/11/2001, 2001.

7. Zhipeng;, T.; Liang;, M.; Zhitao;, L.; Songzhi, Y. Preparation Method Of N-(3,4Dichlorobenzyl)Hexamethylene Tetra-Ammonium Chloride. CN107286168 (A), 2017-1024, 2017.

8. Basace, Y., Delepine reaction of acetylenic compounds. Bulletin de la Société chimique de France. 1971, (4), 1468-1472

9. Minghu, W.; Guichun, Y.; Zuxing, C., Bis(1-Benzyl-3,5,7-Triaza-1Azoniatricyclo[3.3.1.13,7] Decane) Peroxodisulfate: A Mild and Efficient Oxidation of Organic Compounds Under Anhydrous and Aprotic Conditions. Synth. Commun. 2000, 30 (17), 3127-313110.1080/00397910008086921.

10. Geisler, K.; Pfeiffer, W.-D.; Müller, C.; Nobst, E.; Bulka, E.; Langer, P., Synthesis and Functionalization of 4-Halomethyl-1,3-selenazoles. Synthesis 2003, 2003 (08), 1215$122010.1055 / \mathrm{s}-2003-39406$.

11. Maligres, P. E.; Waters, M. S.; Weissman, S. A.; McWilliams, J. C.; Lewis, S.; Cowen, J.; Reamer, R. A.; Volante, R. P.; Reider, P. J.; Askin, D., Preparation of a clinically investigated ras farnesyl transferase inhibitor. J. Heterocycl. Chem. 2003, 40 (2), 229$24110.1002 /$ jhet.5570400206.

12. Ding, Y., Improved synthesis of olanexidine hydrochloride. Zhongguo Yaowu Huaxue Zazhi 2005, 15 (6), 369-370

13. Xinglong;, L.; Guangxia;, X.; Qingshan;, K.; Shiyan;, C.; Hai, X. Method for preparing 5-aminolevulinate 2019.

14. E, P. C. Hexamethylenetetramine Adducts With Haloacetic Acid Esters. US3624253A, 1971.

15. Brandänge, S.; Rodriguez, B., A Convenient Route to 3-Pyrroline Utilizing the Delépine Reaction. Synthesis 1988, 1988 (04), 347-34810.1055/s-1988-27571.

16. Rajesh, T.; Azeez, S. A.; Naresh, E.; Madhusudhan, G.; Mukkanti, K., Practical One-Pot and Large-Scale Synthesis of N-(tert-Butyloxycarbonyl)-3-pyrroline. Org. Process Res. Dev. 2009, 13 (3), 638-64010.1021/op8003037.

17. Snyder, H. R.; Demuth, J. R., Some Secondary Amines in the Sommelet Reaction. J. Am. Chem. Soc. 1956, 78 (9), 1981-198410.1021/ja01590a061.

18. Günther, M.; Lategahn, J.; Juchum, M.; Döring, E.; Keul, M.; Engel, J.; Tumbrink, H. L.; Rauh, D.; Laufer, S., Trisubstituted Pyridinylimidazoles as Potent Inhibitors of the Clinically Resistant L858R/T790M/C797S EGFR Mutant: Targeting of Both Hydrophobic Regions and the Phosphate Binding Site. J. Med. Chem. 2017, 60 (13), 5613563710.1021/acs.jmedchem.7b00316.

19. Malova Krizkova, P.; Hammerschmidt, F., On the Configurational Stability of Chiral Heteroatom-Substituted [D1]Methylpalladium Complexes as Intermediates of Stille and Suzuki-Miyaura Cross-Coupling Reactions. Eur. J. Org. Chem. 2013, 2013 (23), 5143514810.1002/ejoc.201300439. 